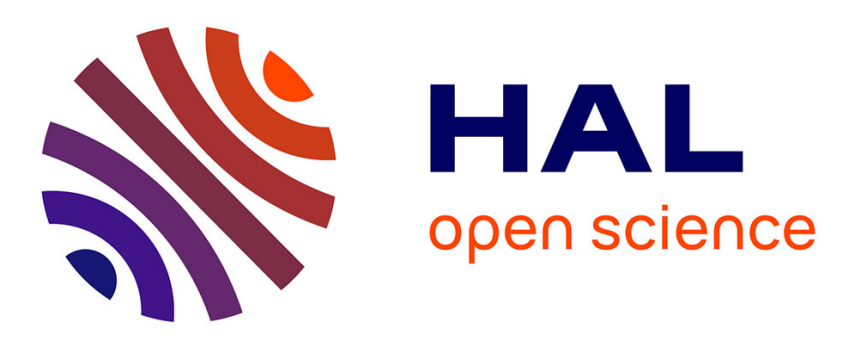

\title{
Bayesian inversion of the Martian structure using geodynamic constraints
}

Mélanie Drilleau, Henri Samuel, Attilio Rivoldini, Mark Panning, Philippe

Lognonné

\section{- To cite this version:}

Mélanie Drilleau, Henri Samuel, Attilio Rivoldini, Mark Panning, Philippe Lognonné. Bayesian inversion of the Martian structure using geodynamic constraints. Geophysical Journal International, 2021, 226 (3), pp.1615-1644. 10.1093/gji/ggab105 . hal-03429374

\section{HAL Id: hal-03429374 https://hal.science/hal-03429374}

Submitted on 15 Nov 2021

HAL is a multi-disciplinary open access archive for the deposit and dissemination of scientific research documents, whether they are published or not. The documents may come from teaching and research institutions in France or abroad, or from public or private research centers.
L'archive ouverte pluridisciplinaire HAL, est destinée au dépôt et à la diffusion de documents scientifiques de niveau recherche, publiés ou non, émanant des établissements d'enseignement et de recherche français ou étrangers, des laboratoires publics ou privés. 


\title{
Bayesian inversion of the Martian structure using geodynamic constraints
}

\author{
Mélanie Drilleau ${ }^{\oplus},{ }^{1,2}$ Henri Samuel ${ }^{\oplus},{ }^{1}$ Attilio Rivoldini, ${ }^{3}$ Mark Panning ${ }^{4,5}$ and \\ Philippe Lognonné ${ }^{1}$ \\ ${ }^{1}$ Institut de Physique du Globe de Paris, CNRS, Université de Paris, 1 rue Jussieu, 75005 Paris, France. E-mail: melanie.drilleau@isae-supaero.fr \\ ${ }^{2}$ Institut Supérieur de l'Aéronautique et de l'Espace ISAE-SUPAERO, 10 Avenue Edouard Belin, 31400 Toulouse, France \\ ${ }^{3}$ Royal Observatory of Belgium, Brussels, Belgium \\ ${ }^{4}$ Jet propulsion Laboratory, Pasadena, CA, USA \\ ${ }^{5}$ California Institute of Technology, Pasadena, CA, USA
}

Accepted 2021 March 11. Received 2021 January 22; in original form 2020 July 17

\begin{abstract}
SUMMAR Y
The ongoing InSight mission has recently deployed very broad band seismometers to record the Martian seismic activity. These recordings constitute the first seismic data set collected at the surface of Mars. This unique but sparse record compels for the development of new techniques tailored to make the best use of the specific context of single station-multiple events with several possible ranges of uncertainties on the event location. To this end, we conducted sets of Markov chain Monte Carlo inversions for the 1-D seismic structure of Mars. We compared two inversion techniques that differ from the nature of the parametrization on which they rely. A first classical approach based on a parametrization of the 1-D seismic profile using Bézier curves. A second, less conventional approach that relies on a parametrization in terms of quantities that influence the thermochemical evolution of the planet (mantle rheology, initial thermal state and composition), which accounts for 4.5 Gyr of planetary evolution. We considered several combinations of true model parameters to retrieve, and explored the influence of the type of seismic data (body waves with or without surface waves), the number of events and their associated epicentral distances and uncertainties, and the presence of potential constraints on Moho depth inferred from independent measurements/considerations (receiver functions and gravity data). We show that due to its inherent tighter constraints the coupled approach allows a considerably better retrieval of Moho depth and the seismic structure underneath it than the classical inversion, under the condition that the physical assumptions made in coupled approach are valid for Mars. In addition, our tests indicate that in order to constrain the seismic structure of Mars with InSight data, the following independent conditions must be met: (1) The presence of surface waves triggered by an internal source to constrain the epicentral distance. (2) The presence of just a few well-localized impact sources, with at least one located at close epicentral distance $\left(<5^{\circ}\right)$ to illuminate independently the crust and the mantle. In addition to providing tighter constraints of Mars seismic structure, geodynamically constrained inversions allow one to reconstruct the thermochemical and rheological history of Mars until present. Therefore, even with a relatively small amount of large events and in absence of surface waves, constraining the present-day structure and long-term evolution of the red planet remains possible through the use of tailored hybrid inversion schemes.
\end{abstract}

Key words: Composition and structure of the mantle; Inverse theory; Numerical modelling; Probability distributions; Planetary interiors; Computational seismology.

\section{INTRODUCTION}

Planetary exploration and characterization rely on data to improve understanding the present-day deep structure, the origin and the long-term evolution of planetary bodies. In the case of Mars, a number of space missions have sent orbiters, landers and rovers to measure magnetic (Acuña et al. 1998) and gravity fields (e.g., 
Smith et al. 1999; Konopliv et al. 2006), as well as spectra to determine the surface composition (Boynton et al. 2007; Blake et al. 2013), topography (Zuber et al. 1992), landscape (McEwen et al. 2007) and other geodetic quantities such as the precession rate (e.g. Folkner et al. 1997) and solid tides (Yoder et al. 2003). While gravity data and measured geodetic quantities can provide important constraints on the internal structure of the planet (Wieczorek \& Zuber 2004; Rivoldini et al. 2011; Baratoux et al. 2014), they constitute only a first-order picture of the interior of the planet (Van Hoolst \& Rivoldini 2014). As on Earth and on other explored planetary bodies with seismic activity, seismology remains the most suitable approach to sample the detailed structure of a planet (Lognonné \& Mosser 1993). For example, our current knowledge about the seismic velocity structure of the lunar interior was considerably improved from analysis of the data collected by the seismometers deployed during the Apollo missions. 1-D seismic models of the lunar structure were inferred from the recording of direct $P$ - and $S$ wave arrival times (e.g. Toksoz et al. 1974; Nakamura 1983; Khan \& Mosegaard 2002; Lognonné et al. 2003), and more recently by including more global geophysical information (e.g. mass, moment of inertia, love numbers, electromagnetic sounding data) in addition to seismic data (e.g. Khan et al. 2004; Gagnepain-Beyneix et al. 2006; Khan et al. 2014; Matsumoto et al. 2015; Garcia et al. 2011, 2019).

The exploration of Mars is going along the same path with the ongoing InSight mission, whose main motivation is to investigate its deep structure with the deployment for the first time of a shortperiod seismometer and three very broad-band seismometers to record its seismic activity (Lognonné et al. 2019; Banerdt et al. 2020). This achievement has led to the first Martian seismic catalog (InSight Mars SEIS Data Service 2019; InSight Marsquake Service 2020).

Along with data comes their interpretation, and in the case of Insight, the first Martian seismic recordings have not been short of surprises. Pre-InSight mission studies have developed inversion techniques to constrain the seismic structure of the red planet. Most of these approaches were based on some a priori knowledge and expectations of the planet structure and composition, inspired by pre-existing knowledge (Smrekar et al. 2019). Our knowledge of the interior structure and the evolution of terrestrial bodies is primarily based on the abundant data sets available for the Earth, and secondarily from the much more limited data set collected on the Moon. In particular, these have guided teams of planetary seismologists to develop inversion methods based on the propagation of surface waves (Panning et al. 2015; Khan et al. 2016; Panning et al. 2017) to constrain the 1-D deep structure of Mars. A critical aim of these methods was to handle probabilistic approaches in order to deal with the large ineluctable uncertainties associated with data scarcity and sparsity. However, while a fair number of events have been detected since the deployment of the seismic instruments at the surface of Mars so far, no surface waves have been clearly identified (Giardini et al. 2020; Lognonné et al. 2020). This does not mean that none of the recorded events have triggered measurable surface waves, nor that any future event of shallow (e.g. impacts Daubar et al. 2018) or deep (Marsquakes) origin will not be able to trigger surface waves. However, at this point methods that rely on surface waves to constrain the interior of Mars cannot be used.

In spite of its pioneering initiative, the InSight seismic experiment is bound to provide a precious but sparse seismic data set. Indeed, even though the number of recorded events grows rapidly (Giardini et al. 2020), strong magnitude events remain rare and the 'network' consists of a single station. Overcoming such limitations requires the development of new approaches along with their optimal tuning, to make the best use of the available InSight seismic data set, in the light of what we have learned since the deployment of the instruments. This is the reason why testing and improving seismic inversion methods using synthetic data remains crucial, even though the InSight data set is already available.

In this context, the resolving power of the current and upcoming seismic record can be improved by supplementing prior constraints. In this spirit, a novel geodynamically constrained inversion of seismic data has recently been developed (Drilleau et al. 2020). This represents the first attempt to infer the Martian seismic structure using a hybrid inversion strategy (Drilleau et al. 2020). In addition to constraining the seismic structure of Mars, this approach infers additional key quantities that are poorly constrained (i.e. mantle rheological parameters) because they cannot be simply directly measured, and allows the planet's thermochemical history to be reconstructed, which also happens to be one of the main objective of the InSight mission.

While the above approach has proven to be potentially powerful to constrain the seismic structure of Mars, the geodynamically constrained inversion described in Drilleau et al. (2020) has been tested on a single prescribed synthetic data set and a single seismic structure to retrieve. However, the potential of the method could not be fully revealed nor generally improved through the inversion of a single data set, and the performances of the inversion may strongly vary according to the considered input data (i.e. the use of surface waves and/or body waves, the source location, the number of seismic events). In this paper, we aim towards a step further to the initial study of Drilleau et al. (2020), which focused on seismic velocity profiles, using a single data set, and considering a single input seismic structure. Here, our objective is to investigate in detail the limits and strengths of the method by testing different data sets and different input seismic structures, in order to assess to what extent the geodynamic parameters could be constrained with available and upcoming data. With the current data only including $P$ and $S$ arrivals for several events all below magnitude 4 (Banerdt et al. 2020; Giardini et al. 2020), it is very challenging to constrain interior structure. We propose to test methods that may be able to constrain mantle structure either with one larger event with measurable surface wave dispersion, or one to a few impact events located by orbital imagery in order to have them fully tested when the upcoming events will be recorded.

To address these questions, we performed a comparative testing of geodynamically constrained seismic inversions with a more conventional inversion approach. We applied these distinct types of inversions to several hypothetical data sets, by considering several combinations of true model parameters to retrieve, different types of seismic data (body waves with or without surface waves), by varying the number of events and their associated epicentral distances and uncertainties, and by accounting for additional potential constraints on Moho depths inferred from independent measurements/considerations (receiver functions and gravity data).

The paper is organized as follows: Section 2 summarizes the methodologies involved to perform the inversions (forward and inverse problems), Section 3 presents the inversion experiments we performed to test our two approaches, Section 4 discusses the inversion experiments results and Section 5 summarizes the main findings of this study. 


\section{INVERSION METHODOLOGY}

\subsection{Forward problem}

The forward problem consists in computing seismic data (body waves and/or Rayleigh waves arrival times) from a knowledge of the interior structure of the crust, mantle and core. Two main approaches are considered. First, a classical approach, for which the models are parametrized in seismic velocity as a function of depth. Secondly, a coupled geodynamic-seismic approach, parametrized by 'geodynamic parameters' (these terms will refer throughout to parameters that can influence the dynamic thermochemical evolution of the planet and include thermal, rheological, or thermodynamic quantities). As noted in Drilleau et al. (2020), the strength of the classical approach is that it can provide extreme models (in the sense that the seismic velocities along the inverted radial structure do not depend on constraints from mineral physics and thermodynamics, which allows for more flexibility in the model sampling) if the composition, and/or the geodynamic parameters are variable along the wave path, and can violate $a$ priori mineralogical, physical and thermodynamic constraints and bounds that may not apply to the planet. However, such models do not take into account constraints from mineral physics, moment of inertia or the thermal evolution of the planet, which limits their resolving power. Conversely, the geodynamic-seismic approach allows the application of tight constraints on velocity structure with a relatively limited data set (as in the case of Mars), by producing consistent velocity models through the entire planet, in contrast to the classical models which give some constraints only at the depths where the data are sensitive. Therefore, the geodynamic-seismic approach can be powerful given good prior constraints on the thermochemical structure of Mars.

\subsubsection{Classical approach}

This approach is mainly based on the work of Drilleau et al. (2013, 2020). The 1-D $V_{S}$ models are parametrized with two layers in the crust, and with six Bézier points in the mantle down to the core, which are interpolated using $C_{1}$ polynomial Bézier curves. The $V_{P} / V_{S}$ ratio is also parametrized using Bézier points, located at the same depths than the $V_{S}$ Bézier points. The inverted parameters are the depth of the two layers in the crust and their corresponding $V_{S}$ values, the depth and $V_{S}$ values of the Bézier points in the mantle, the $V_{P} / V_{S}$ ratios in the two layers of the crust, and the $V_{P} / V_{S}$ values of the Bézier points in the mantle. The Bézier points are randomly located between the Moho depth and the top of the core. Due to the lack of seismic data traveling down to the core, the core radius is not inverted for and fixed to $1750 \mathrm{~km}$. In addition, this approach does not enforce the matching of the planet's mass and moment of inertia. The details on the a priori assumptions on the inverted parameters are given in Section 2.2.3.

Each sampled model relies on the ray tracing algorithm of Shearer (2009) to compute body wave arrival times. The surface wave velocity dispersion curves are computed using the MINEOS software package (Masters et al. 2011), and are then converted to arrival times using the epicentral distance value.

\subsubsection{Coupled geodynamic-seismic approach}

Forward model: a parametrized thermochemical and thermodynamic approach. We follow the same approach described in (Drilleau et al. 2020), which consists of a Markov chain Monte Carlo
(McMC) joint inversion of body waves and surface-wave seismic data, where the modelling of Mars' thermochemical history is embedded in the forward problem using a parametrized convection model. The resulting present-day thermochemical state of the planet is used to compute the seismic structure of Mars in terms of body wave profiles assuming a compositional, mineralogical and thermodynamic model for Mars. The parametrized model for Mars considers that the planet is divided into several concentric and spherically symmetric envelopes radially ordered as illustrated in Figs 1(a) and (b):

(i) A convecting iron-sulfur liquid core (Rivoldini et al. 2011).

(ii) An adiabatic convecting silicate mantle (with top and bottom thermal boundary layers).

(iii) A stagnant lithospheric lid, of thickness, $D_{l}$, which includes a crust of thickness $D_{c r}$, enriched in heat-producing elements.

The thermochemical evolution is computed following the approach in Samuel et al. (2019) and references therein. It reproduces the evolution of a Mars-sized stagnant-lid planet in spherical geometry well, both in the case of a transient or steady-state evolution, including the effects of complexities such as temperature and pressure-dependent mantle viscosities and the presence of a crust whose thickness progressively evolves via melt extraction at shallow pressure. The evolution of the layered planet is computed by numerically integrating a set of coupled differential equations expressing the conservation of internal energy $U$ within each convecting envelope, $i$, in the following form:

$\frac{d U_{i}}{d t}=H_{i}+F_{i}^{\mathrm{bot}}-F_{i}^{\mathrm{top}}$,

which includes on the right hand side internal heat production by radioactive elements and latent heating/cooling effects, and heat fluxes $F$ across planetary envelopes, respectively. The heat fluxes depend essentially on the temperature contrast between each envelope, and on mantle viscosity. Therefore, the viscosity of the Martian mantle plays an important role, and is assumed to depend on both temperature, $T$, and pressure, $P$, following an Arrhenius relationship (Karato \& Wu 1993):

$\eta(T, P)=\eta_{0} \exp \left(\frac{E^{*}+P V^{*}}{R T}-\frac{E^{*}+P_{\mathrm{ref}} V^{*}}{R T_{\mathrm{ref}}}\right)$,

where $E^{*}$ and $V^{*}$ are the effective activation energy and activation volume (i.e. the sensitivity of viscosity to temperature and pressure, respectively), $R$ is the gas constant, $T_{\text {ref }}=1600 \mathrm{~K}$ and $P_{\text {ref }}=3 \mathrm{GPa}$ are the reference temperature and pressure at which viscosity equals the reference viscosity, $\eta_{0}$. The effective activation volume and energy can account for viscous deformation in the diffusion creep regime, or in the dislocation creep regime (Christensen 1983; Plesa et al. 2015; Kiefer \& Li 2016; Samuel et al. 2019).

The temperature profile in the purely conducting lithospheric layer (which includes the crust, see Fig. 1b) is obtained by integrating the unsteady heat balance equation with radially dependent heat sources, density, and thermal conductivity, to account for different values of these parameters in the enriched and buoyant crust and in the depleted residual lithosphere. The lithospheric and crustal thicknesses evolve as a function of the time-dependent thermochemical state of the planet.

Following Samuel et al. (2019, and references therein) the crust is assumed to progressively form via mantle melt extraction at pressure smaller than 7.4 GPa (Ohtani et al. 1998). Beyond this pressure the melt is no longer assumed to be positively buoyant. The crustal production rate that is directly proportional to the volume 
(a) Model envelopes at present-day

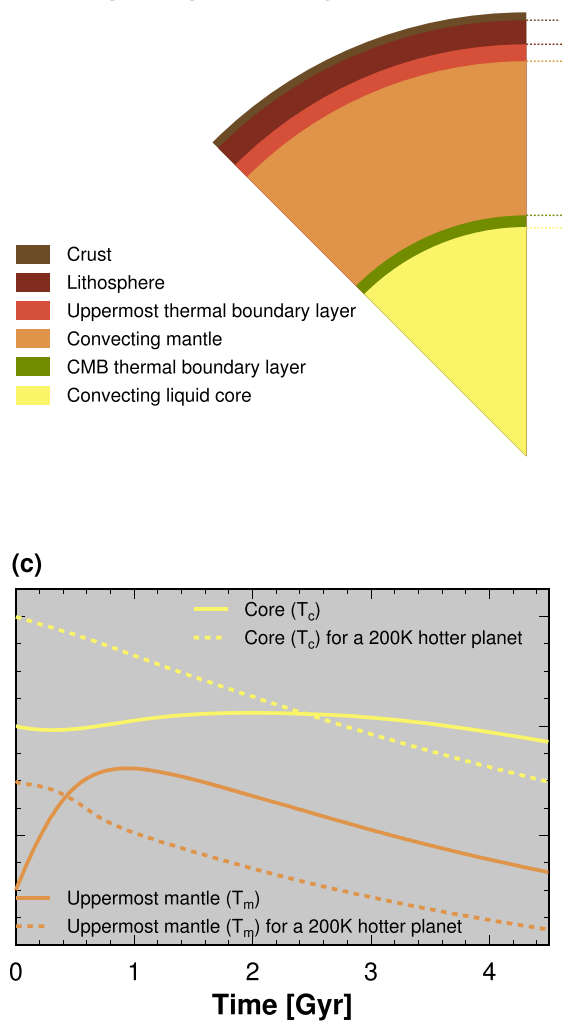

(b)

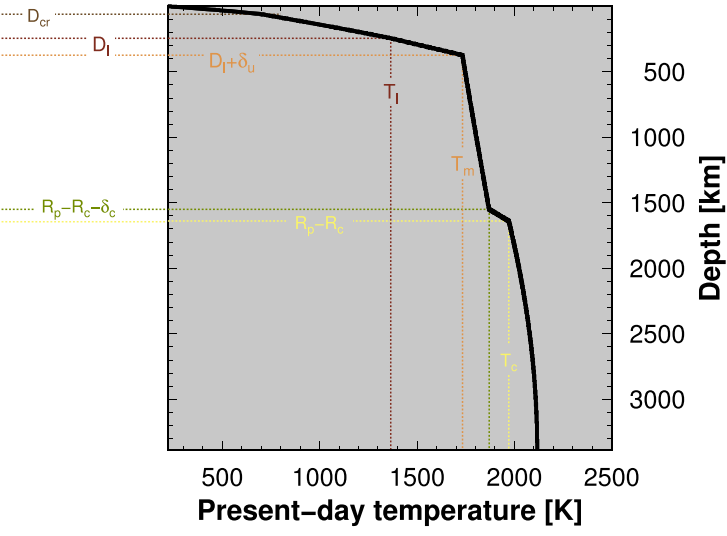

(d)

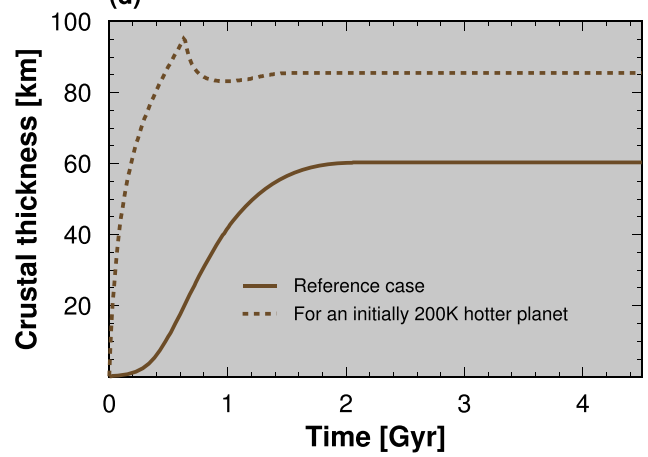

Figure 1. Example of the thermochemical evolution of a Mars-like planet, for $E^{*}=150 \mathrm{~kJ} \mathrm{~mol}^{-1}$, and $V^{*}=3 \mathrm{~cm}^{3} \mathrm{~mol}^{-1}$. Present-day structure (a) and areotherm (b) resulting from $4.5 \mathrm{Gyr}$ of evolution. (c) Time-evolution of mantle and core temperature below the top and bottom thermal boundary layers, respectively for a reference case with initially $T_{m}=1700 \mathrm{~K}$ and $T_{c}=2000 \mathrm{~K}$ (plain curves) and a case with $T_{m}=1900 \mathrm{~K}$ and $T_{c}=2100 \mathrm{~K}$ (dotted curves). (d) Time evolution of crustal thicknesses.

of mantle melt at shallow pressure and to the magnitude of convective mantle velocities is computed at each time step (Samuel et al. 2019), where we account for the progressive effect of melt extraction on the solidus (Schumacher \& Breuer 2006). The crust and the mantle are mutually coupled, because crust formation depletes the mantle in heat-producing elements, which progressively reduces shallow mantle temperatures and melting. The effect of the cooling of the mantle depleted in heat-producing elements also results in a thickening of the lithosphere and influences the planet heat loss. In addition, crustal formation influences the heat balance due the latent heat consumed or released upon melting and crystallization at shallow depths. The crustal enrichment factor (i.e. the amount of heat-producing elements in the crust relative to that of the primitive mantle) is fixed to 10 , as suggested by constraints on elastic thickness (Thiriet et al. 2018), and compatible with gammaray spectrometer data (Hahn et al. 2011). Despite these constraints, larger values of crustal enrichment cannot completely be ruled out. For simplicity, we chose not to invert for the crustal enrichment here.

Finally, present-day areotherms are obtained by evolving the parametrized thermochemical calculations for 4.5 Gyr (Fig. 1b).

The parameters that we infer with the McMC inversion procedure are: (1) The mantle rheology through the effective activation energy $\left(E^{*}\right)$, and reference viscosity $\left(\eta_{0}\right) ;(2)$ the initial thermal state through the temperature below the lithosphere $\left(T_{m_{0}}\right)$. We chose to invert for these parameters because they exert a considerable influence on the thermal evolution and because they remain poorly constrained. The initial CMB temperature $\left(T_{c_{0}}\right)$ is tied to the mantle initial thermal state, $T_{m_{0}}$, to allow for the presence of an early Martian thermal dynamo, as suggested by magnetic data (e.g. Acuña et al. 1998; Connerney et al. 2004): $T_{c_{0}}=T_{m_{0}}+300 \mathrm{~K}$. Note that within reasonable bounds we verified than modifying this value of $300 \mathrm{~K}$ does not change our inversion results. The core radius is kept constant at $1750 \mathrm{~km}$, which falls well withing the range of plausible values for Mars. While such value for Mars remains known within a rather large uncertainty on the order of $\sim \pm 100 \mathrm{~km}$ (Van Hoolst \& Rivoldini 2014; Smrekar et al. 2019), its influence within its plausible range on the thermal evolution and the resulting present-day seismic structure is small because it would correspond to a modest variation in volume, which would not significantly affect the thermal evolution of the planet. In addition the seismic phases in the data set considered here do not account for core phases and core-reflected phases. Constraints on Mars-Phobos thermal-orbital history favour a relatively narrow range for the effective activation volume for the mantle of Mars: $V^{*}=0-4 \mathrm{~cm}^{3} \mathrm{~mol}^{-1}$ (Samuel et al. 2019). Therefore, we set $V^{*}=3 \mathrm{~cm}^{3} \mathrm{~mol}^{-1}$. We tested the influence of inverting also for a variable $V^{*}$ and found no significant differences with respect to the case where $V^{*}$ is fixed (A).

The resulting present-day thermal profiles for the thermochemical model (Fig. 1b) are used to compute seismic velocity profiles. The computation of seismic velocities from thermal profiles is performed using a thermodynamic model in the mantle beneath the crust via the Perple_X Gibbs energy minimization software (Connolly 2005), that relies on formulation and the database of Stixrude \& Lithgow-Bertelloni (2005). Perple_X is used to compute tabulated values for density, $P$ and $S$ waves seismic velocities at various 
mantle pressure and temperatures and mantle composition, which self-consistently accounts for phase transitions. These values are then interpolated using cubic splines along the temperature profiles resulting from the thermochemical evolution modelling. We proceed with interpolation rather than on-the-fly calculation of density and seismic velocities for computational efficiency purposes. Nevertheless, the interpolation errors are not significant. We assume a mantle bulk composition of Taylor (2013). Note that in Section 4.2 we evaluated the effect of different compositions on our inversion results. The seismic velocities in the crustal layer are decoupled from the thermal state because the mineralogy and the structure of the crustal layers is not well understood and likely not in thermodynamic equilibrium, which prevents the use of static thermodynamic models. Therefore, we considered several crustal layers of variable thickness, in which the body wave velocities and density are uniform, with the uppermost part consisting of a 2-km-thick bedrock layer with a reduced density of $1900 \mathrm{~kg} \mathrm{~m}^{-3}$, and reduced seismic velocities [with $V_{P}$ and $V_{S}$ being set to 0.6 and 0.5 times the value of the corresponding velocities in the layer directly below it (Smrekar et al. 2019)]. In the inversion process, the seismic velocities in the crust can vary by multiplying the obtained seismic velocities by two factors that we call crustal $V_{P}$ and $V_{S}$ factors (see Section 2.2.3).

Links between the thermochemical evolution and the present-day seismic structure. Different thermal histories result in a variety of thermochemical structures, notably in terms of crustal and lithospheric thicknesses, or in mantle and core thermal states. This is illustrated in Figs 1(c) and (d), which show the influence of the initial mantle and core temperatures on the thermal evolution of the planet and on the evolution of the crustal thickness. The two cases that are displayed only differ by their $T_{m_{0}}$ and $T_{c_{0}}$ values. The hottest case yields a colder present-day mantle (Fig. 1c). This apparent contradiction [often referred to as the 'thermostat effect' (Schubert et al. 1979)] is due to the fact that a hotter planet is less viscous (see eq. 2) and therefore cools down more efficiently. However, an initially hotter planet produces larger amounts of melt at shallow depths, leading to a thicker crust (Fig. 1d).

The differences in the thermal profiles affect the local phase equilibria and the thermoelastic properties of each phase and thus result in distinct seismic velocity profiles. Fig. 2 illustrates those differences for the mantle, where three different thermochemical histories were considered: A 'reference' case and a 'hot' and 'cold' end-member case.

Each of these three evolutions yield different Moho and lithospheric depths, and distinct temperature profiles in the convecting mantle (Figs $2 \mathrm{a}-\mathrm{c}$ ). These result in the presence of different phases in distinct proportions as a function of mantle depth (Figs 2d-f). This leads to different seismic velocity profiles (Figs g-i), where phase transition occur at different depths (in particular the OlivineRingwoodite-Wadsleite transition). The temperature and pressure differences between the different cases can result in different stable phases at a given depth. For instance, the hot and reference profiles (Figs 2e-f) do not allow for significant fractions of Ringwoodite or HP-clinopyroxene to occur but have Wüstite present, contrary to the cold profile (Fig. 2d). Overall, this illustrates how different values of governing parameters (here, temperature associated with mantle rheology) yield different Martian thermochemical histories, and result in distinct seismic velocity profiles.

Consequently, instead of varying independently the values of the density, or body wave velocities along a given profile depth during the inversion process, we sample the model space by varying the values of the governing parameters mentioned above (mantle rheology and initial thermal state) that control Mars' thermochemical history. This approach yields the density and seismic velocity profiles for the core, the mantle and the crust.

As mentioned above, unlike the mantle, the structure of the crust, the layering and its seismic velocities are not computed from its chemical composition by Gibbs energy minimization because its composition is not well known, likely not in thermodynamic equilibrium, and heavy altered by surface processes. For this reason, we directly vary and invert for the crustal seismic velocity structure (both in terms of layering, and in terms of the values of seismic velocities within each crustal layer) instead of deriving it purely from thermal and mineral physics considerations because our mineralogical model does not apply for crustal conditions, and our approach only constrains crustal thickness and its density. Only evolutions that lead to a convective mantle are retained (i.e. cases for which the mantle is sub-critical will be excluded, because crater counting (Hartmann et al. 1999) indicates the presence of recent volcanism on Mars, which suggests that the interior of Mars is convectively active. The seismic velocity profiles were allowed to be shifted by \pm 5 per cent in order to account for uncertainties in the thermochemical and mineralogical models. In practice, in the inversion algorithm, the obtained mantle velocity profiles are multiplied by two factors randomly sampled between 0.95 and 1.05 , which we call mantle $V_{P}$ and $V_{S}$ factors (see Section 2.2.3).

In spite of the prior constraints induced by the geodynamic framework, eq. (2) suggests trade-offs between governing parameters (i.e. temperature and rheology) implying that several characteristics of a given thermal history (crustal and lithospheric thicknesses, mantle and core temperatures and their corresponding seismic profiles) can be obtained via different combination of thermal and rheological parameters. This is illustrated in Figs 3 and 4 that display present-day quantities characterizing the thermochemical state of the planet (uppermost core and mantle temperatures, lithospheric and crustal thicknesses) as a function of mantle rheology ( $E^{*}$ and $\eta_{0}$ ) for a planet initially cold or hot (Figs 3 and 4). Not only strong trade-offs between the planet thermochemical state and mantle rheology exist, but these trade-offs change considerably with the initial thermal state of the planet.

Nevertheless, in addition to allow for a better self-consistency than varying independently seismological parameters along the inversion process (Moho depth, seismic velocities, etc.), the built-in geodynamic frame significantly reduces the parameter space, by accounting for the interdependencies between various quantities (e.g. temperature, composition, rheology) and their influences on crustal thickness. This approach can also constrain the value of physical quantities inaccessible to direct measurements, such as the rheology of the Martian mantle, and provides the entire history of the planet associated with each model.

As for the classical approach (Section 2.1.1), the algorithms from Shearer (2009) and Masters et al. (2011) are used to compute body waves and surface waves arrival times, respectively, from the seismic velocity profiles.

\subsection{Inverse problem}

The inverse problem consists in retrieving the model parameters (seismic velocities or geodynamic parameters) from the seismic waves arrival times. This problem is however ill-posed: different structure models can yield similar seismic waves arrival times. The ill-posed nature of the problem is amplified by the presence of noise. Due to the ill-posed nature of the problem, inversions based on least 

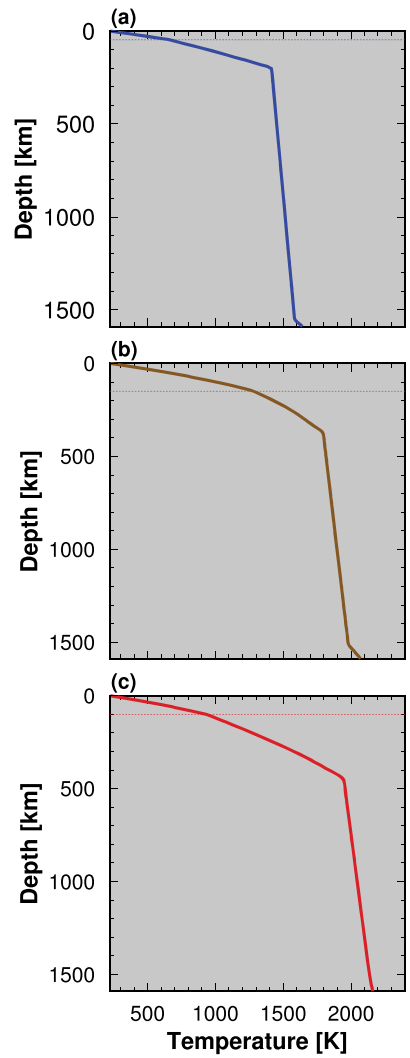

(d)

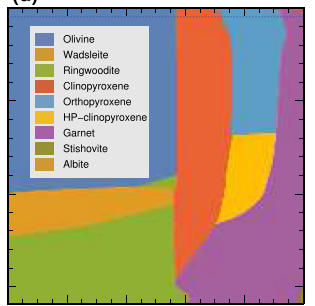

(e)

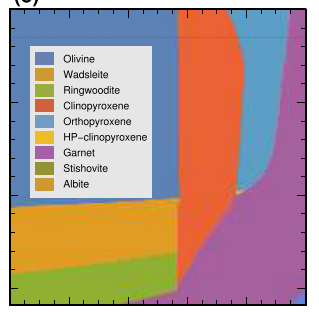

(f)

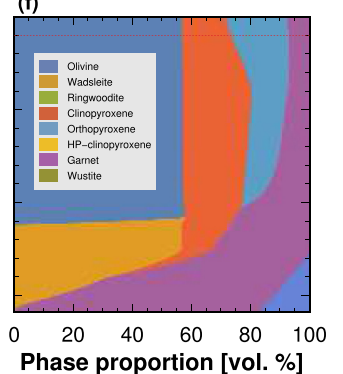

(g)

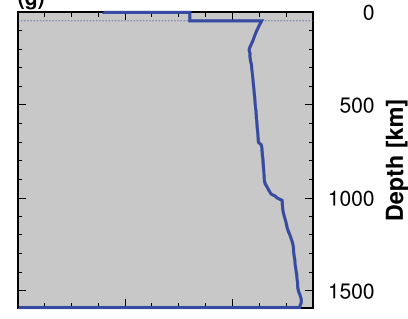

(h)

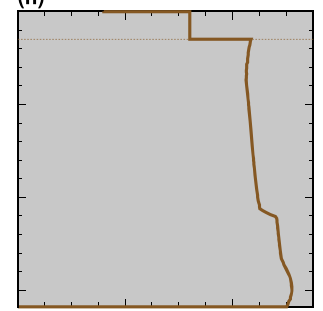

(i)

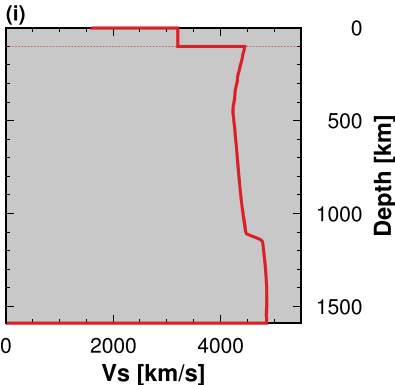

Figure 2. Effect of different governing parameters on the present-day seismic profiles, resulting in a cold (a) and a hot (c) present-day areotherms, relative to a reference profile (b). These profiles are associated with different mineralogies (d-f), and therefore with distinct shear wave velocities (g-i). Dotted horizontal lines indicate the Moho depth. Here the crust is assumed to be composed of a single homogeneous layer (where our mineralogical model does not apply) overlaid by a thin bedrock layer. See text for further details.

squares or grid search in the absence of regularization are not well suited to estimate the model uncertainties, in the sense that the parameter space should be sampled at high resolution. Instead, we used a Bayesian approach based on the McMC method (Mosegaard \& Tarantola 1995; Tarantola 2005). The probabilistic inversion is more suitable to explore the parameter space, includes a priori information, provides reliable estimates of the uncertainties, and is independent on the starting model. We followed the probabilistic procedure developed by Drilleau et al. (2013), and used in Panning et al. (2015, 2017), Garcia et al. (2019) and Drilleau et al. (2020).

\subsubsection{The McMC algorithm}

This section briefly outlines the fundamentals of the Bayesian inversion, based on the McMC method, detailed in Mosegaard \& Tarantola (1995) and Tarantola (2005). Let us denote by $\mathbf{p}$ our model parameters and $\mathbf{d}$ the data, respectively. The data are related to the parameters through the equation, $\mathbf{d}=A(\mathbf{p})$, where the non-analytic and non-linear operator $A$ represents the forward problem discussed in Section 2.1. The solutions of the inverse problem are described by the posterior probabilities $P(\mathbf{p} \mid \mathbf{d})$ that the parameters are in a configuration $\mathbf{p}$ given the data are in a configuration $\mathbf{d}$. The parameter space is sampled according to $P(\mathbf{p} \mid \mathbf{d})$. Bayes' theorem links the prior distribution $P(\mathbf{p})$ and the posterior distribution $P(\mathbf{p} \mid \mathbf{d})$,

$P(\mathbf{p} \mid \mathbf{d})=\frac{P(\mathbf{d} \mid \mathbf{p}) P(\mathbf{p})}{\sum_{\mathbf{p} \in \mathcal{M}} P(\mathbf{d} \mid \mathbf{p}) P(\mathbf{p})}$, where $\mathcal{M}$ denotes all the possible configurations in the parameter space. $P(\mathbf{p})$ defines the prior distribution, that is the set of possible models that reduce the configuration space and represents our state of knowledge. $P(\mathbf{d} \mid \mathbf{p})$ represents the likelihood of data observation given a model $\mathbf{p}$, which is related to the misfit of the predicted data to the observed data.

To sample the posterior distribution (eq. 3) in the model space, we use the Metropolis-Hastings algorithm (Metropolis et al. 1953; Hastings 1970). This algorithm samples the model space in a random fashion, with a sampling density proportional to the unknown (target) posterior probability density function, and ensures that lowprobability areas are sampled less extensively. This algorithm relies on a randomized decision rule, which accepts or rejects the proposed model according to its fit to the data, and if the model satisfies the prior conditions.

\subsubsection{Misfit function}

The likelihood function $P(\mathbf{d} \mid \mathbf{p})$ is a function of the misfit $S[\mathbf{d}$, $A(\mathbf{p})]$, which determines the difference between the observed data d and the computed synthetic data $A(\mathbf{p})$. Minimizing the misfit function is equivalent to maximizing the likelihood function, that is $P(\mathbf{d} \mid \mathbf{p}) \propto \exp [-S(\mathbf{d}, A(\mathbf{p}))]$. Since the origin time $\left(t_{0}\right)$ of the seismic event is considered here as unknown (because the InSight seismic network consists of a single seismic station), we use differential times relative to the $P$-wave phase arrival. Given the complexity of Martian seismograms (Giardini et al. 2020) the data could be suspected of containing outlier(s), which could most probably be 

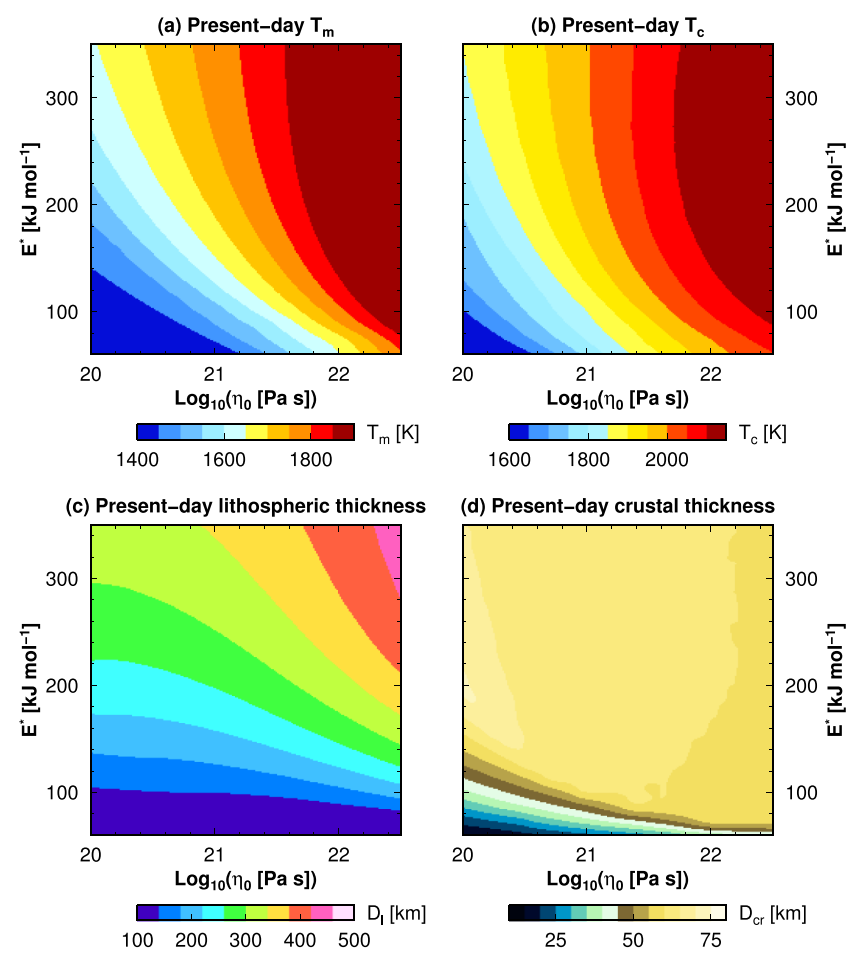

Figure 3. Trade-offs between rheological governing parameters and their influences on the present-day thermochemical structure of a Mars-like planet as a function of mantle rheological parameters (reference viscosity, $\eta_{0}$, and effective activation energy, $\left.E^{*}\right)$. The results correspond to initial mantle and core temperatures of 1700 and $2000 \mathrm{~K}$, respectively, a core radius of $1750 \mathrm{~km}$, and a mantle activation volume of $3 \mathrm{~cm}^{3} \mathrm{~mol}^{-1}$. Present-day values for (a) temperature under the lithosphere, (b) core temperature at the CMB, (c) lithospheric thickness and (d) crustal thickness.
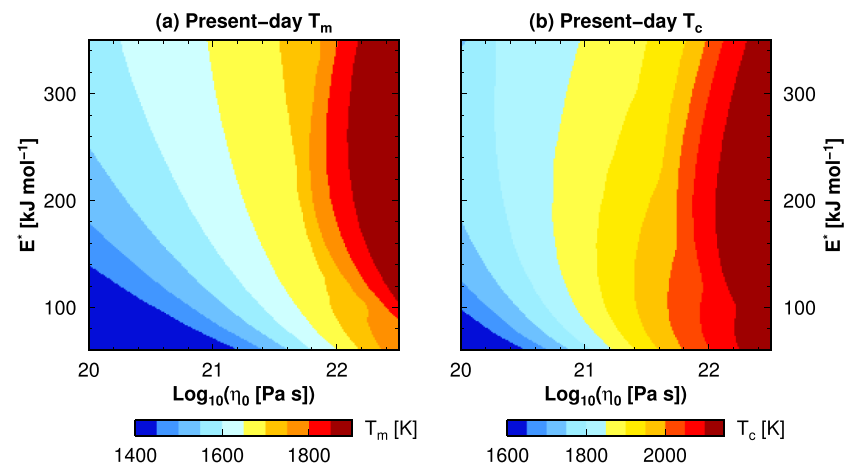

(c) Present-day lithospheric thickness

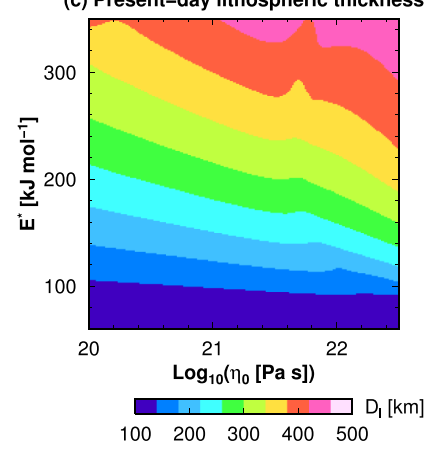

(d) Present-day crustal thickness

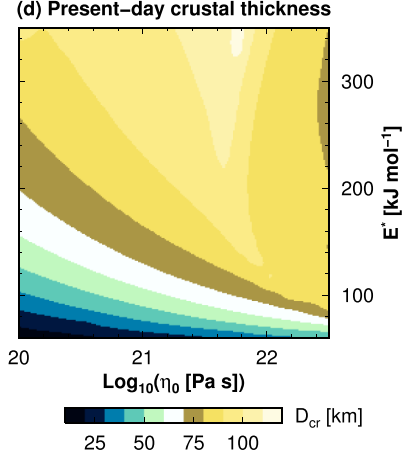

Figure 4. Trade-offs between thermal governing parameters and their influences on the present-day thermochemical structure of a Mars-like planet, as a function of the initial mantle $\left(T_{m_{0}}\right)$ and core $\left(T_{c_{0}}\right)$ initial thermal state. Same as Fig. 3 for an initially hotter planet: of $T_{m}=1900 \mathrm{~K}$ and $T_{c}=$ $2200 \mathrm{~K}$. the result of an erroneously read arrival time. A $L_{1}$ norm is thus preferred to a $L_{2}$ norm, because it is considered to be more robust to outliers (Khan \& Mosegaard 2002; Tarantola 2005). The cost function is then defined as follows:

$$
\begin{aligned}
S(\mathbf{d}, A(\mathbf{p}))= & \frac{\left|\left(t_{\mathrm{S}}^{\text {obs }}-t_{\mathrm{P}}^{\text {obs }}\right)-\left(t_{\mathrm{S}}^{\text {calc }}-t_{\mathrm{P}}^{\text {calc }}\right)\right|}{\sigma_{\mathrm{S}}+\sigma_{\mathrm{P}}} \\
& +\sum_{N}^{i=1} \frac{\left|\left(t_{R_{i}^{o b s}}-t_{\mathrm{P}}^{\text {obs }}\right)-\left(t_{R_{i}^{c}}^{\text {calc }}-t_{\mathrm{P}}^{\text {calc }}\right)\right|}{\sigma_{R i}+\sigma_{\mathrm{P}}},
\end{aligned}
$$

where $S[\mathbf{d}, A(\mathbf{p})]$ quantifies the misfit between the observed and computed differential arrival times $t_{\mathrm{S}}-t_{\mathrm{P}}$, and the sum of the misfits between the observed and computed differential arrival times $t_{R}-$ $t_{\mathrm{P}}$ at each of the $N$ periods, taking into account the error bars $\sigma_{\mathrm{P}}$, $\sigma_{\mathrm{S}}$ and $\sigma_{R}$ on $P, S$ and Rayleigh waves arrival times, respectively. Superscripts refer to observations (obs) and computed data (calc). Note that when dealing with body waves only (Section 3.2), only the first term on the right-hand side of the above equation is considered.

\subsubsection{Prior information}

The solutions are sampled according to a set of rules named prior, which define the set of possible models reducing the configuration space and representing our state of knowledge. We choose to compare two different parametrizations, one using seismic velocity (classical inversion), and another one using physical assumptions (geodynamically constrained inversion).

The parameters of the two different models are randomly sampled within relatively broad parameter spaces (Table 1). In particular, the representative range of seismic Martian interior models described in Smrekar et al. (2019), constrained by geodetic data, geochemical and thermal considerations, is included within the prior bounds. The classical models make fewer assumptions on the depth of the structural discontinuities, whereas in the case of the geodynamically constrained models, the Moho depth is directly estimated in the forward problem from the sampled geodynamic parameters. In order to ensure the presence of a velocity jump at the Moho depth for the classical models, we require that the shear wave velocities $\left(V_{S}\right)$ in the first Bézier point in the mantle to be larger than the value of $V_{S}$ in the second layer of the two crustal layers. In total, 24 parameters are inverted using the classical approach (Section 2.1.1 and Table 1), while the geodynamically constrained approach requires the inversion of only seven parameters (Section 2.1.2 and Table 1). Upon the relocation of a quake during the structure inversion process (Section 3.1.2), the corresponding epicentral distance is sampled between $0^{\circ}$ and $100^{\circ}$, which yields a supplementary parameter to invert for.

In the McMC algorithms, new models are proposed by randomly perturbing the previously accepted model. Here, the sampling of the parameter space is performed using a continuous proposal function. Let $p_{i}^{t}$ and $p_{i}^{t+1}$ be the $t$ th and the $(t+1)$ th value of a parameter $p$, respectively. Then the subsequent step may be defined as $p_{i}^{t+1}=p_{i}^{t}+w^{i}$, where $w^{i}$ is the $t$ th stepsize, randomly sampled from a normal distribution with a zero mean. A Gaussian probability density distribution, centred at $p_{i}^{t}$ is used to randomly sample $p_{i}^{t+1}$, which explains why the parameter distributions are all Gaussian (Table 1).

Figs 5(a), (c) and (b), (d) show the a priori distributions of $V_{S}$ and the $V_{P} / V_{S}$ ratio as a function of depth for the geodynamic and classical approaches, respectively, given the prescribed conditions detailed in Table 1. Both the a priori assumptions and the sampling of the models lead to non-uniform distributions. For both models, 
Table 1. Synthesis of the inverted parameters and the prior bounds considered for classical and geodynamically constrained models.

\begin{tabular}{lccl}
\hline Classical models & & & \\
Description & Quantity & Value/range & Distribution \\
\hline Depth of layer 1 & 1 & $0-10 \mathrm{~km}$ & Gaussian \\
Moho depth (depth of layer 2) & 1 & $10-130 \mathrm{~km}$ & Gaussian \\
$V_{S}$ in layer 1 & 1 & $0.5-2.5 \mathrm{~km} \mathrm{~s}^{-1}$ & Gaussian \\
$V_{S}$ in layer 2 & 1 & $2.0-3.5 \mathrm{~km} \mathrm{~s}^{-1}$ & Gaussian \\
$V_{S}$ in the mantle & 12 & $3.5-5.0 \mathrm{~km} \mathrm{~s}^{-1}$ & Gaussian \\
$V_{P} / V_{S}$ in layer 1 & 1 & $1.5-2.2$ & Gaussian \\
$V_{P} / V_{S}$ in layer 2 & 1 & $1.5-2.2$ & Gaussian \\
$V_{P} / V_{S}$ in the mantle & 6 & $1.5-2.2$ & Gaussian \\
Geodynamically constrained models & & & \\
Description & Quantity & Value/range & Distribution \\
$T_{m 0}$ & 1 & $1800-2000 \mathrm{~K}$ & Gaussian \\
$T_{c 0}$ & 1 & $T_{c 0}-T_{m 0}=300 \mathrm{~K}$ & depends on $T_{m 0}$ \\
$E^{*}$ & 1 & $60-500 \mathrm{~kJ} / \mathrm{mol}$ & Gaussian \\
$\eta_{0}$ & 1 & $10^{20}-10^{22.5} \mathrm{~Pa} \mathrm{~s}$ & Gaussian \\
$V^{*}$ & 1 & $3.0 \mathrm{~cm}^{3} \mathrm{~mol} \mathrm{l}^{-1}$ & Fixed \\
$R_{c}$ & 1 & $1750 \mathrm{~km}$ & Fixed \\
Crustal $V_{S}$ factor & 1 & $0.5-1.5$ & Gaussian \\
Crustal $V_{P}$ factor & 1 & $0.5-1.5$ & Gaussian \\
Mantle $V_{S}$ factor & 1 & $0.95-1.05$ & Gaussian \\
Mantle $V_{P}$ factor & 1 & $0.95-1.05$ & Gaussian \\
\hline
\end{tabular}
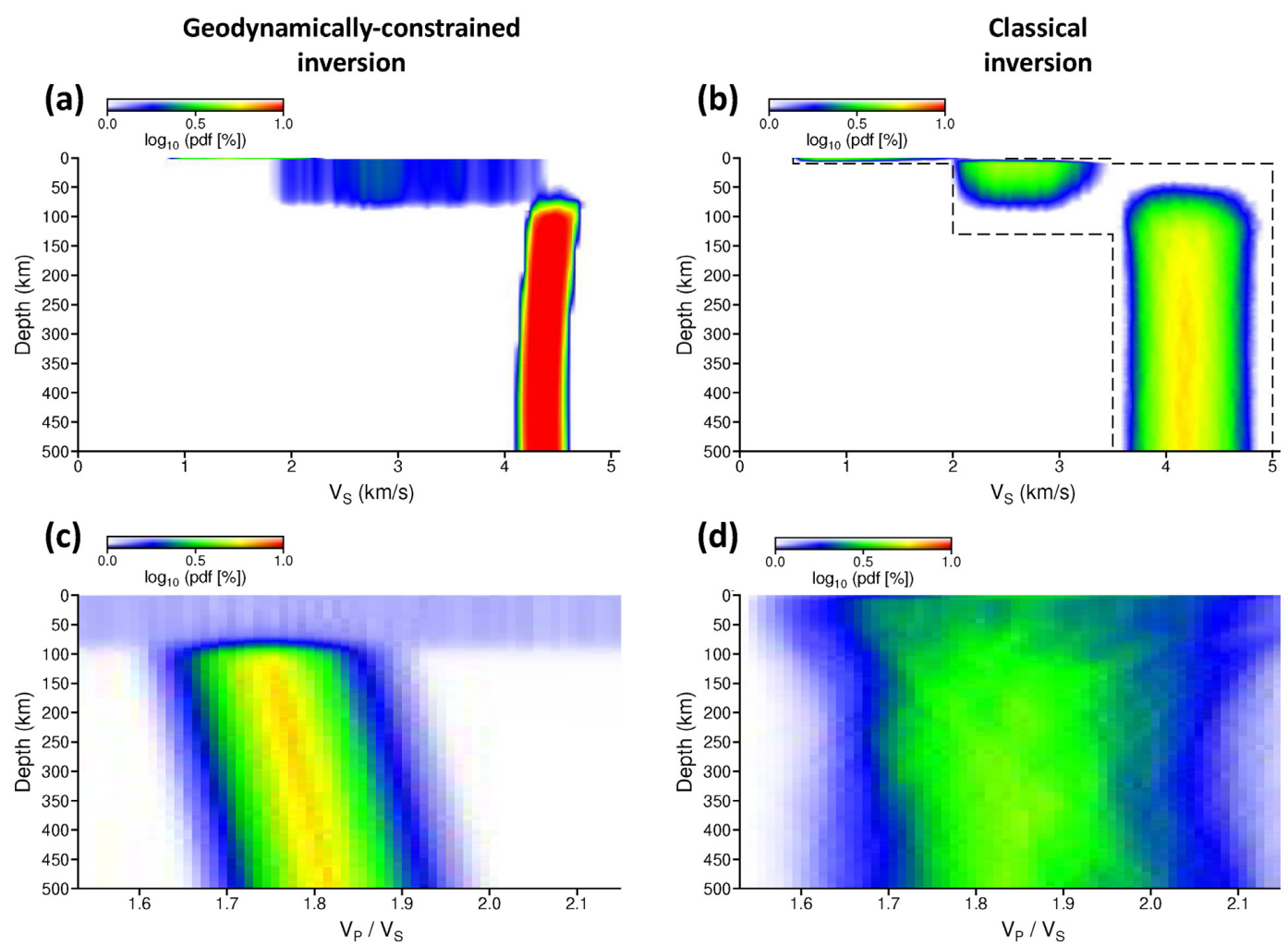

Figure 5. A priori probability density functions (pdfs) in logarithmic scale of the 1-D $V_{S}$ profiles, and the $V_{P} / V_{S}$ ratio, for the geodynamic (a, c) and classical (b, d) approaches, considering that all the sampled models which are in good agreement with a priori information detailed in Table 1 are accepted. These are the priors of the inversions detailed in Section 3. Red and blue colours show small and large probabilities, respectively. The pdf is computed by counting the number of sampled profiles in each of the cases. The discretization is $1 \mathrm{~km}$ for depth, and $0.05 \mathrm{~km} \mathrm{~s}^{-1}$ and 0.01 for $V_{S}$ and the $V_{P} / V_{S}$ ratio, respectively. For a given depth, the sum of the pdf over all the parameter intervals is equal to 100 per cent. Dashed lines in (b) shows the prior bounds of classical models, as detailed in Table 1.

the center of the parameter space is better sampled than the bounds in the crust and in the mantle, due to the choice of a Gaussian probability density distribution that samples the models, as stated above. In the mantle, the $V_{S}$ and the $V_{P} / V_{S}$ ratio a priori distributions of the geodynamic models (Figs $5 \mathrm{a}$ and c) are narrowed compared to the classical models (Figs $5 \mathrm{~b}$ and d), due to the physical assumptions used in the forward problem (Section 2.1.2). The 
$V_{P} / V_{S}$ ratio decreases as a function of depth for the geodynamic models (Fig. 5c), because the increasing temperature with depth in the mantle produces a larger decrease of $V_{S}$ than $V_{P}$ as a function of depth. In contrast, the $V_{P} / V_{S}$ ratio of the classical models (Fig. 5d) are allowed to decrease or to increase as a function of depth. It is worth noting that the classical models cover a range of $V_{S}$ and $V_{P} / V_{S}$ values that is larger than the realistic properties of the expected rocks. However, considering a large parameter space for the inverse problem ensures that no acceptable region in the solution space is missed.

\subsubsection{Practical implementation details}

Along the McMC process, samples (models) are usually kept to compute the a posteriori probability density function, only after the parameters have stabilized around some values, which indicates that the algorithm has converged. The stages prior to and after convergence are named 'burn-in stage' and 'stationary stage', respectively. We perform a parallel two-steps inversion scheme to speed-up the convergence towards the stationary stage. During a first step that is assigned to the burn-in stage, a broad exploration of the model space is performed by randomly perturbing the parameters using wide Gaussian proposal distributions, which are necessary to loose the memory of the initial configuration (starting model). To allow the algorithm to sample a sufficient number of extrema in the model space, we run 96 independent Markov chains in parallel. One of the major issues with McMC exploration is the difficulty to predict the number of iterations required to achieve statistical convergence. Upon testing, we found that the convergence during this stage is reached after at most 300 iterations. The main indicators of this convergence are the significant decrease of the misfit and the stabilization of the parameter values. The starting model for each chain is randomly chosen within the prior, and thus each chain follows a different path in the model space. To ensure a broad exploration of the model space, each Markov chain is associated with a unique random seed. At each iteration, the synthetic data computed from the given configuration are tested against the real data by computing the misfit (eq. 4). The best-fitting model is then determined for each chain and are sorted in ascending order. To discard the chains that might not have converged, the 72 first configurations with the smallest misfits of these 96 best-fitting models are selected to be the starting models of the second step (or stationary stage), which will be used to compute the statistics. After this first stage of broad parameter space exploration, the selected 72 independent chains are run in parallel for 3000 iterations during the second step, sampling the parameter space with narrower Gaussian proposal distributions. Reducing the Gaussian proposal distributions ensures this time to preserve most of the characteristics of the starting model, which may have resulted in a good data fit. While being efficient, this strategy will result in a sequence of models which tend to be correlated with each other. The occurrence of these inter-dependencies is unfortunate, since both the error analysis and a good spatial resolution require a collection of statistically independent models from the posterior distribution. A way around this problem is to select fewer samples from the set of accepted models in such a way that they constitute a set of independent models. Consequently, the posterior probabilities are constructed by downsampling by a factor of five the number of models sampled during the stationary period, in order to prevent model interdependencies.

\section{INVERSION EXPERIMENTS}

In this section, we present series of inversions in which we vary the type of seismic sources, their number and their location, and the amount of noise, in order to cover a variety of possible scenarios from the optimistic to the most pessimistic case. This allows testing and comparing the resolving power and the limitations of the classical and the coupled approach in different configurations.

\subsection{Inversions using surface waves and body waves}

We first present and discuss the results inverting both surface waves and body waves. We considered here an optimistic scenario, where $P$ and $S$ waves arrivals are clearly visible on the seismogram, and where the surface wave energy is comprised between 15 and $50 \mathrm{~s}$. Using such a period range, the surface waves should be sensitive to the structure down to the upper mantle of Mars along the sourcereceiver path. We assigned $1 \mathrm{~s}$ uncertainty on the picking of body waves arrival times, and we consider 3 per cent uncertainty on the surface waves arrival times. Note that the inversion parameters can be seen as averaged quantities over the great circle path between source and receiver. The implications for the interpretation of the inversion results with respect to a 3-D structure will be discussed in Section 4.

\subsubsection{Constrained location}

To estimate to what extent the geodynamic parameters could be retrieved in the case of an optimistic scenario, we first consider that the quake location is known. We inverted synthetic data for a single event located at a distance of $75^{\circ}$ and at a depth of $80 \mathrm{~km}$, and we considered 12 different true combinations of $\eta_{0}$ and $E^{*}$ to estimate the impact of the true model configuration on the inversion results. For all inversions, the initial value of the uppermost mantle temperature, $T_{m_{0}}$, for the true model was set to $1900 \mathrm{~K}$. The results are shown in Fig. 6. Because of the complex shape of the posterior distribution arising from strong trade-offs between the parameters (Fig. 4), typically employed statistical measures such as means and covariances were found to be inadequate descriptors. Instead, we present the solution in terms of a large collection of models sampled from the posterior probability density. All the models shown are able to match the arrival times within their uncertainty bounds.

The results in Fig. 6(a) clearly highlight the non-uniqueness of the solution, and validate our approach consisting in the sampling of a large number of models, instead of considering a unique model corresponding to the lowest misfit value. For small true values of $E^{*}$ and $\eta_{0}\left(E^{*}=150 \mathrm{~kJ} \mathrm{~mol}^{-1}\right.$ and $\eta_{0}=10^{20.5} \mathrm{~Pa} \mathrm{~s}$, or $\left.10^{21} \mathrm{~Pa} \mathrm{~s}\right)$, we observe that the configurations leading to small misfit values are aligned along a thin branch, illustrating a strong trade-off between $E^{*}$ and $\eta_{0}$. However, when the sampled values of $\eta_{0}$ are larger than $10^{21.5} \mathrm{~Pa} \mathrm{~s}$, a second branch appears and all the configurations with $E^{*}$ between 50 and $500 \mathrm{~kJ} \mathrm{~mol}^{-1}$ are able to explain the data. For larger true values of $E^{*}$ and $\eta_{0}$, the areas of low misfit values increase. Fig. 6(b) demonstrates that the sampled configurations with low misfit values correspond to seismic velocity models with a crustal thickness close to the value to retrieve. This reveals the dominant influence of crustal thickness on the inversion results. In fact, one can observe a strong similarity between the two branches of low misfit values forming a V-shape in Fig. 6(a), and the isocontours of crustal thickness displayed in Fig. 4 in the same space.

Fig. 7 shows the crustal $V_{S}$ factors as a function of the crustal thickness, for the 12 tested configurations. For all cases, the regions 
(a)
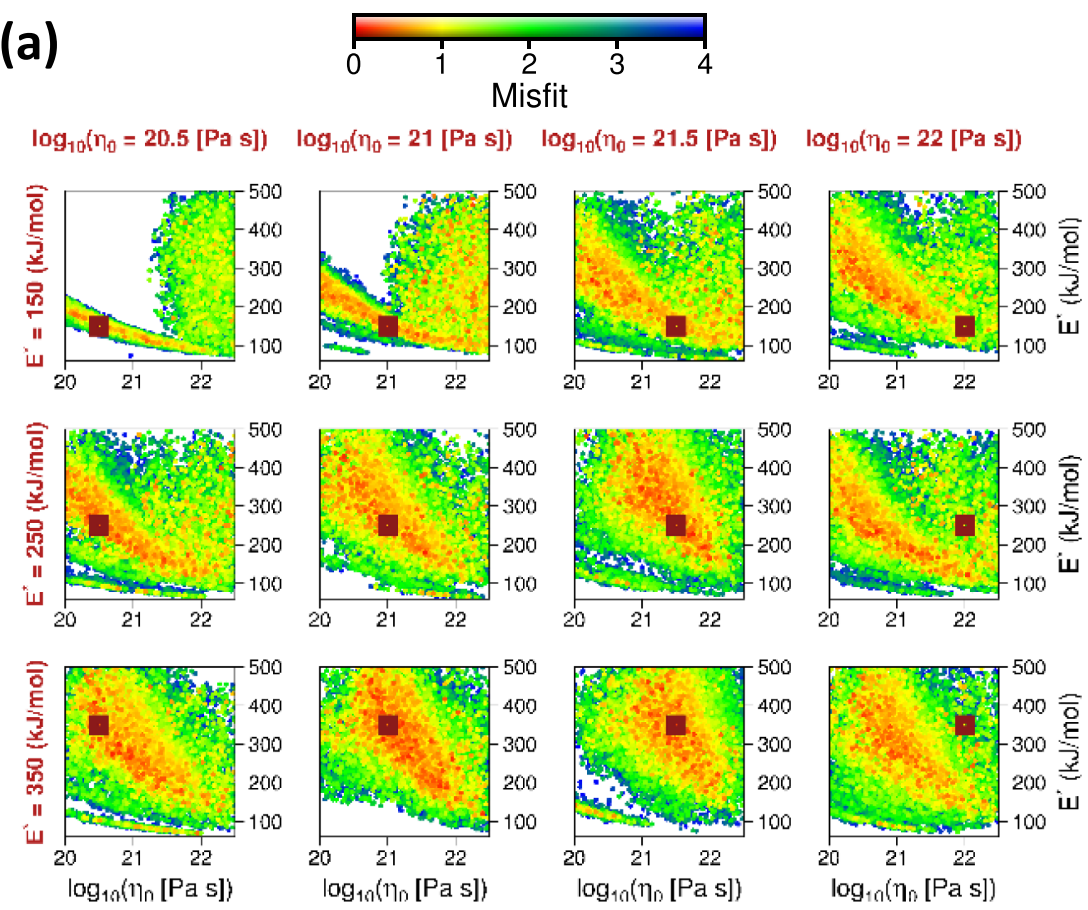

\section{(b)}
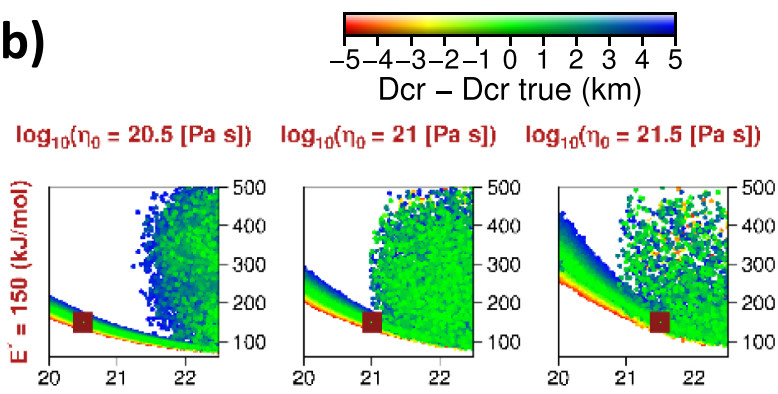

$\log _{10}\left(\eta_{0}=22[\mathrm{~Pa} s]\right)$
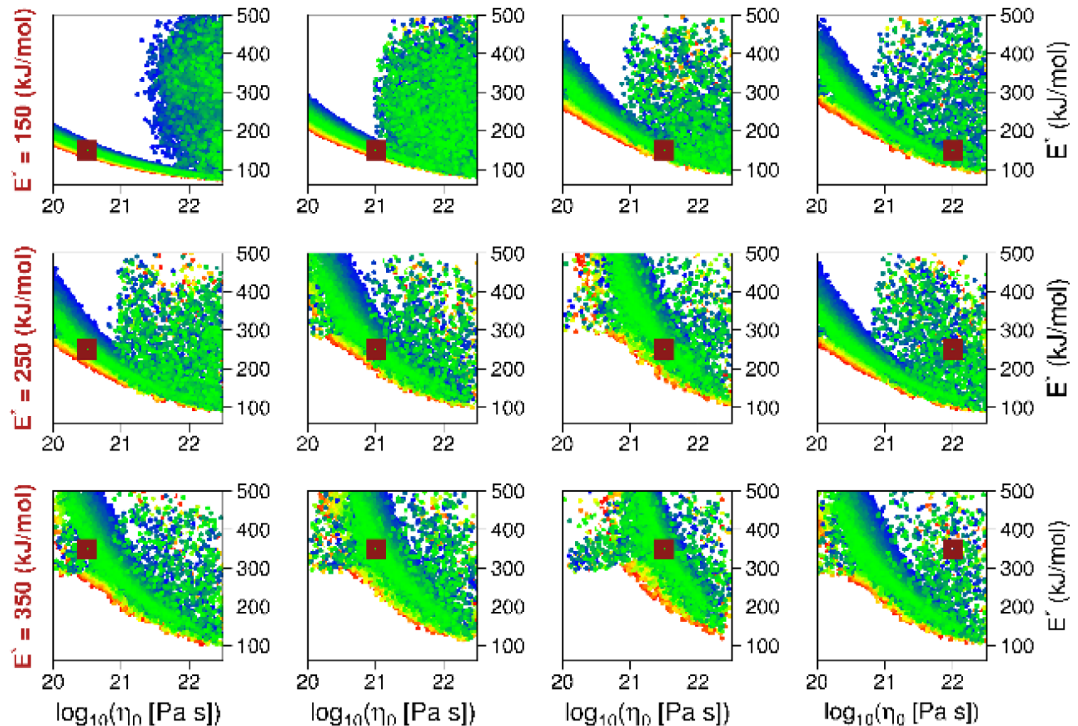

Figure 6. Inversion results using both surface waves and body waves arrival times, and considering a single located event, for 12 different input combinations of $\eta_{0}$ and $E^{*}$. The figures show $\eta_{0}$ [in $\left.\log _{10}(\mathrm{~Pa} \mathrm{~s})\right]$ as a function of $E^{*}$ (in $\mathrm{kJ} \mathrm{mol}^{-1}$ ) for all the models accepted by the Bayesian algorithm (coloured dots). (a) Misfit values. Red and blue colours are small and large misfit values, respectively. (b) Difference between the sampled crustal thickness and the true one, in kilometres. The green colour indicates that the sampled model is close to the input crustal thickness by less than $\pm 1 \mathrm{~km}$. Red and blue colours correspond to thinner and thicker crustal thickness than expected, respectively. The dark red squares correspond to the values to retrieve. The $\eta_{0}$ and $E^{*}$ input values are explicitly written in dark red at the top and on the left of each figure, respectively.

of low misfit values are concentrated around the values to retrieve. We observe a clear correlation between $V_{S}$ in the crust and the crustal thickness (Fig. 6b).

Despite the strong trade-offs between $E^{*}$ and $\eta_{0}$, these results demonstrate that using both surface waves and body waves, for a single located event, would allow one to delimit regions of the parameter space where the solutions are most probable. However, such ability will heavily depend on the unknown geodynamic parameters for Mars, because the spread of possible solutions increases with increasing $E^{*}$ and $\eta_{0}$ values (Fig. 6a).

Another important aspect to consider is the level of noise in the data. We therefore performed the same inversions as those described above except that we introduced random noise into the data within the uncertainty bounds. We tested uncertainty bounds of 1,2 and 

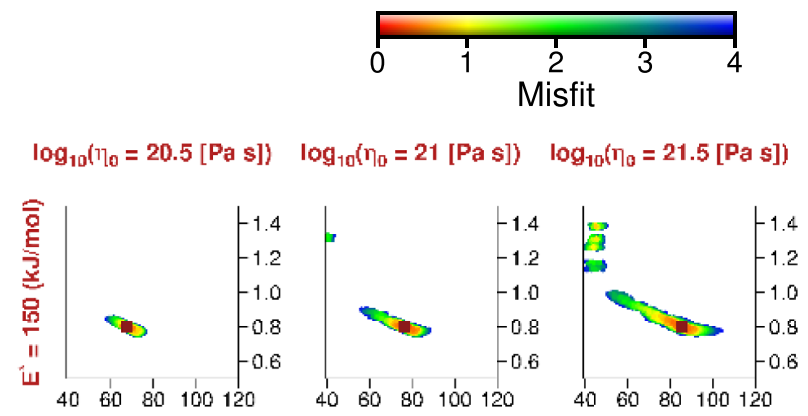

$\log _{10}\left(\eta_{0}=22[\mathrm{Pas}]\right)$
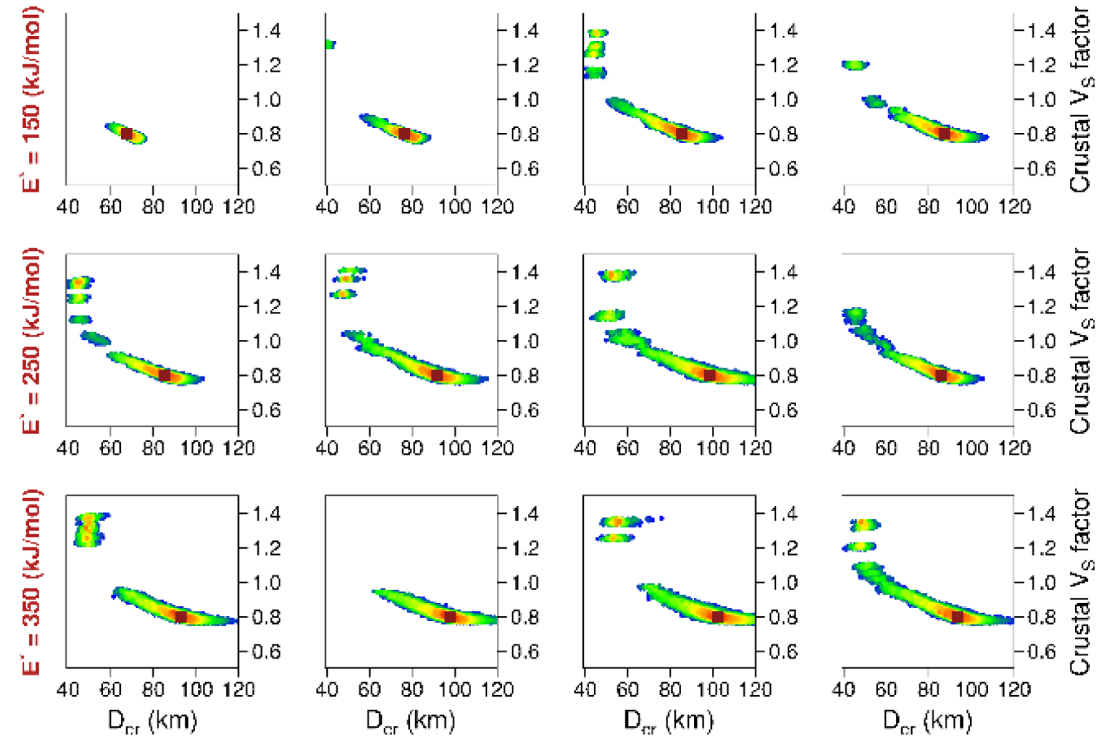

Figure 7. Inversion results using both surface waves and body waves arrival times, and considering a single located event, for 12 different input combinations of $\eta_{0}$ and $E^{*}$. The figures display the crustal thickness (in $\mathrm{km}$ ) as a function of the $V_{S}$ factor in the crust, for all the models accepted by the Bayesian algorithm. Red and blue colours are small and large misfit values, respectively. The dark red squares correspond to the values to retrieve. The $\eta_{0}$ and $E^{*}$ input values are explicitly written in dark red at the top and on the left of each figure, respectively.

3 s for body waves arrival times, and of 3,4 and 5 per cent on the surface waves arrival times. The results (Fig. 8) show that the shape of the distributions for the 10 per cent best models remains similar, but the corresponding areas of low misfit values are larger compared to the case without noise (Fig. 6a).

\subsubsection{Unconstrained location}

We tested the possibility of a data set with unconstrained event location (in terms of both depth and epicentral distance). The inversion results are shown in Figs 9 and 10.

Compared to the results using a fixed location (Fig. 6), the distributions of small misfit values are more scattered within the parameter space, because the introduction of a variable epicentral distance in the inversion process increases the number of combinations that are compatible with the data. Fig. 9(b) reveals a stronger trade-off between the crustal $V_{S}$ factor and the crustal thickness compared to what we observed in Fig. 7. This trade-off unsurprisingly results from the fact that the data are fit equally well when the discontinuity is deeper and $V_{S}$ in the crust is higher, and vice versa. This illustrates the limitation in the resolving power of the data. In spite of this trade-off, the true $V_{S}$ crustal factor and crustal thickness values are located in the area of the low misfit values. The Fig. 10 shows that the output epicentral distances are comprised between $60^{\circ}$ and $100^{\circ}$. A trade-off between the crustal thickness and the epicentral distance is observed, meaning that a close quake considering a thin crust, and a farther quake considering a thick crust, can fit the data equally well. Note that in the absence of depth phase recording, such as pP phases, which reflect from the surface of the planet at a point relatively close to the hypocenter, the depth of the quake cannot be constrained using $P$ and $S$ waves, and surface waves.
These inversion results demonstrate the importance of having a good a priori estimation of the quake location before making some inferences on the geodynamic parameters. Considering a quake large enough to record the third train of surface waves (minor arc plus another trip around the great circle path), determining the distance of the source is a straightforward process (Panning et al. $2015,2017)$. For smaller quakes, the uncertainty on the location may be reduced by comparing the retrieved distance ranges with large potentially active extensive tectonic structure close to InSight on the surface (Giardini et al. 2020).

\subsubsection{Comparison between the classical and the geodynamically constrained approaches}

Fig. 11 depicts a comparison between the classical and the geodynamically constrained inversion output models, as described in Sections 2.1 and 2.2.3, which consider that the event location is known. The true $V_{S}$ seismic profile (in dark red) is the same for both inversions, and was computed using $\eta_{0}=10^{21} \mathrm{~Pa} \mathrm{~s}$ and $E^{*}=$ $150 \mathrm{~kJ} \mathrm{~mol}^{-1}$.

For a better analysis of the results, two different representations are used to investigate the $V_{S}$ distributions. Figs 11(a) and (b) display in grey several models randomly selected in the ensemble models. Mosegaard \& Tarantola (1995) encourage this style of representation, because the classical probability density function representation has a tendency to smooth out the results. Here we consider a very small subset of models ( 15 models), which cannot be used to infer statistical properties. However, this subset is useful to visualize the diversity of the models sampled and to detect the depths where the seismic profile can best be resolved. Figs 11(a) and (b) reveal the regions of the model space where the parameters are constrained. We also represented in Figs 11(c) and (d) the a posteriori probability 


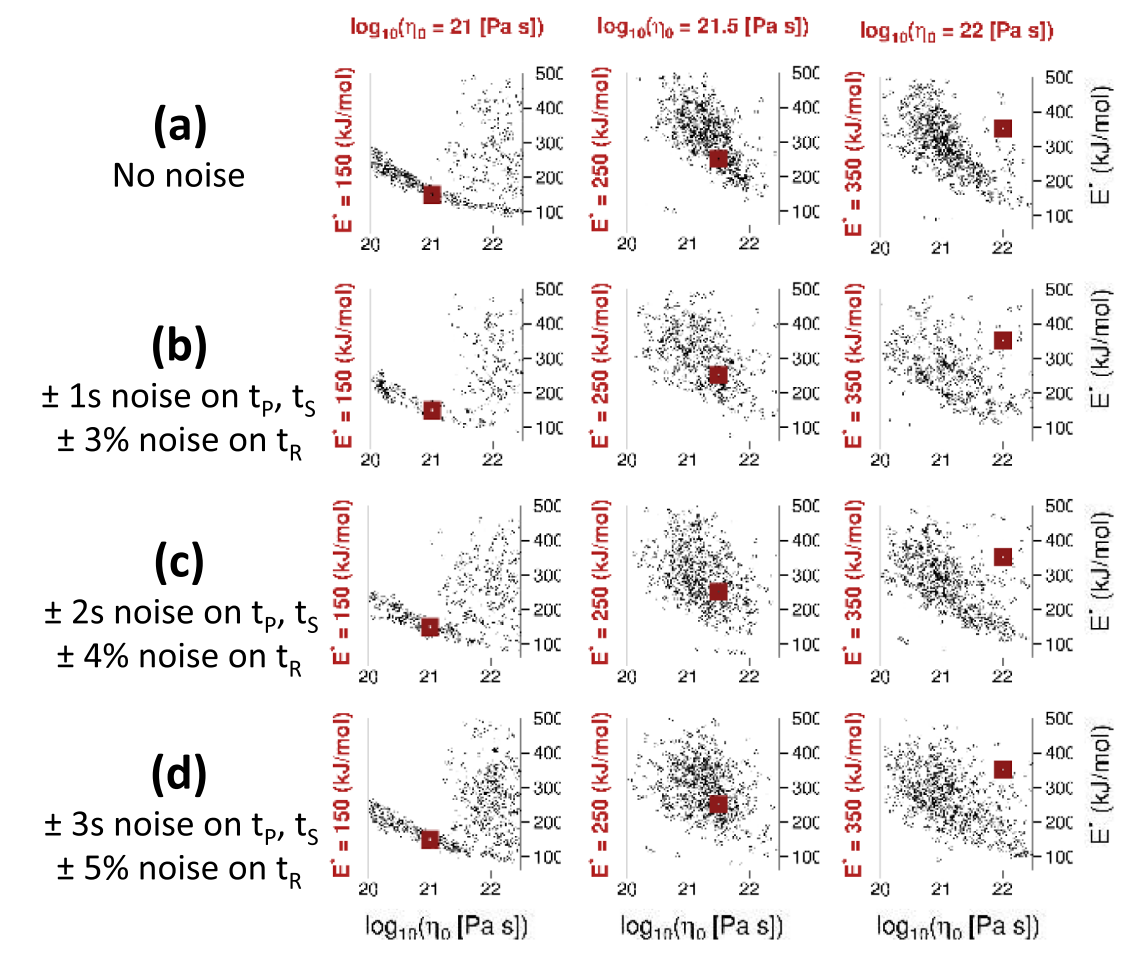

Figure 8. Inversion results using both noisy surface waves and body waves arrival times, and considering a single located event, for three different input combinations of $\eta_{0}$ and $E^{*}$. The figures show $\eta_{0}\left[\right.$ in $\left.\log _{10}(\mathrm{~Pa} \mathrm{~s})\right]$ as a function of $E^{*}\left(\mathrm{in} \mathrm{kJ} \mathrm{mol}^{-1}\right.$ ) for the 10 per cent models with the lowest misfit values. The dark red squares correspond to the values to retrieve. The $\eta_{0}$ and $E^{*}$ input values are explicitly written in dark red at the top and on the left of each figure, respectively. (a) Are the results with no noise. Panels (b), (c) and (d) display the results considering 1,2 and $3 \mathrm{~s}$ noise on the $P$ and $S$ waves arrival times, and 3,4 and 5 per cent on the surface waves arrival times, respectively.

density function (pdf) on $V_{S}$ profiles. The pdfs provide an overview of the most frequently sampled models, and show the additional gain in information obtained through inversion, compared to the $a$ priori distributions (Fig. 5).

We observe that the $V_{S}$ output profiles for the geodynamically constrained inversion are very close to the true model (Figs 11a and $\mathrm{c}$ ). The $1 \sigma$ standard deviation of the pdf corresponds to \pm 2 per cent around the true model in both mantle and the crust. Despite the fact that the output models for the geodynamically constrained inversions are all comparable to the true model, the combination of the corresponding $E^{*}-\eta_{0}$ pairs is far from being unique (Fig. 6, $\eta_{0}$ $=10^{21} \mathrm{~Pa} \mathrm{~s}$ and $E^{*}=150 \mathrm{~kJ} \mathrm{~mol}^{-1}$ ).

The pdf of the classical inversion models shows a $1 \sigma$ standard deviation equal to \pm 5 percent in the crust, and reveals different families of models in the mantle (Figs $11 \mathrm{~b}$ and d). Indeed, some models show a negative velocity gradient comparable to the model to retrieve, while other models display a stronger negative velocity gradient followed by a positive gradient, in addition to models that still do not exhibit a negative velocity gradient (Fig. 11b). These results show that models with distinct characteristics can fit the same data, due to the trade-off between the seismic velocities and the depth of the discontinuities mentioned above. In the mantle, the maximum of the pdf is located in the vicinity of the true profile (Fig. 11d). Nevertheless, the pdf is not unimodal, which confirms that the Bayesian approach is more appropriate than least-squares inversion. In the absence of tight constraints on the velocity structure, the diversity of the $V_{S}$ profiles in the mantle for the classical inversion is also due to the decrease of the sensitivity of Rayleigh waves at these depths, given the period range considered (between 15 and $50 \mathrm{~s})$.
In the crust, the $V_{P} / V_{S}$ ratio is not constrained for both inversions, because the Rayleigh waves are not sensitive to $V_{P}$. Given that the quake is located far from the seismic station $\left(75^{\circ}\right)$, the $P$ wave mostly travels in the mantle and could provide constraints on the $V_{P} / V_{S}$ ratio at the turning point of the ray located in the mantle. For the geodynamically constrained models, $V_{S}$ and $V_{P}$ are linked using common physical assumptions. Consequently, the knowledge of the $V_{P} / V_{S}$ ratio at the turning point of the ray, and a good estimation of the $V_{S}$ profile in the upper mantle are sufficient to retrieve the $V_{P} / V_{S}$ ratio profile in the mantle (Fig. 11e). In contrast, the distribution of the $V_{P} / V_{S}$ ratio of the classical models is very large (Fig. 11f). The highest probability corresponds to $V_{P} / V_{S}$ ranging between 1.7 and 1.9 , and no evidence of a clear decrease with depth is observed.

Even though surface wave data have a weak resolving power for detecting seismic discontinuities, Figs $11(\mathrm{~g})$ and (h) that display the crustal thickness as a function of $V_{S}$ in the crust for all the models selected by the algorithms, indicate that the crustal thickness is well constrained in both cases. The inferred $V_{S}$ pdf is broader for the classical model outputs, due to the trade-off between the seismic velocities in the crust and in the mantle. The geodynamically constrained approach allows the application of tight constraints on the velocity structure in the mantle (Figs 11a and c), which considerably reduces this trade-off. The spread of the $V_{S}$ profiles retrieved with classical method is also due to the fact that they are not built with the same parameterization used to build the true model, which was generated using the geodynamic forward problem (see Section 2.1.2). This also explains why the misfit values are slightly larger than those corresponding to the geodynamically constrained output models (Figs 11g and h). 
(a)
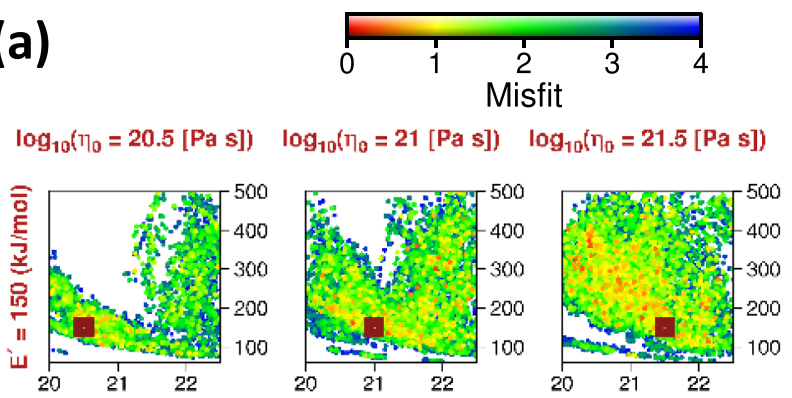

$\log _{10}\left(\eta_{0}=22[\mathrm{~Pa} s]\right)$
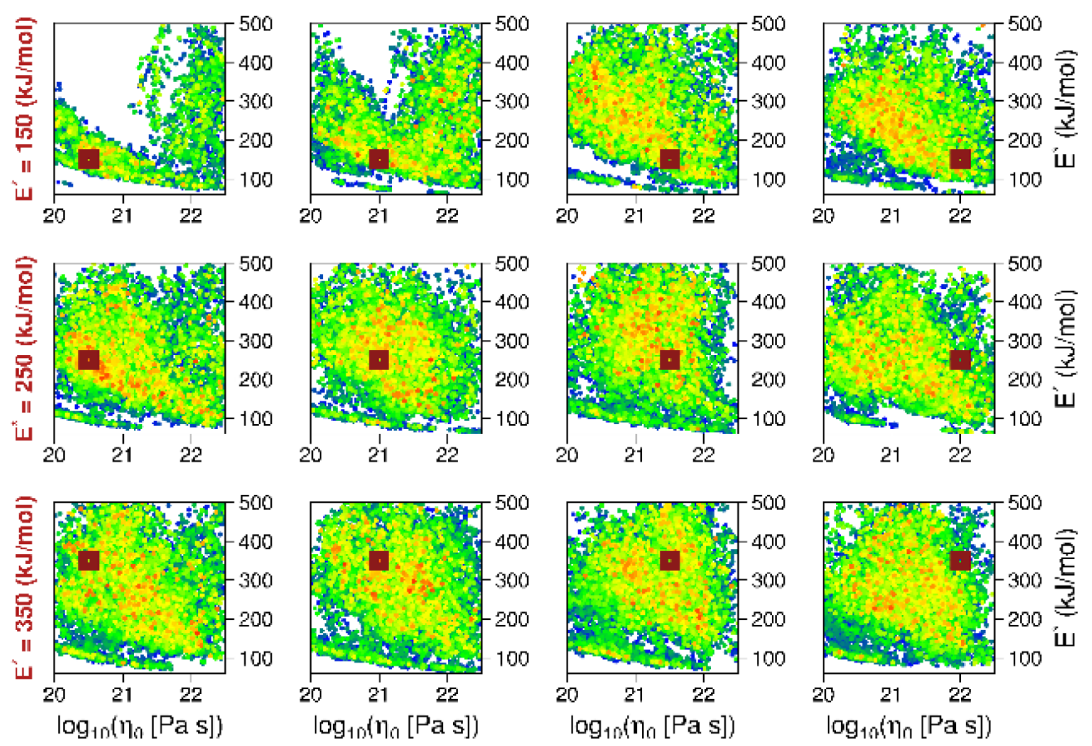

(b)
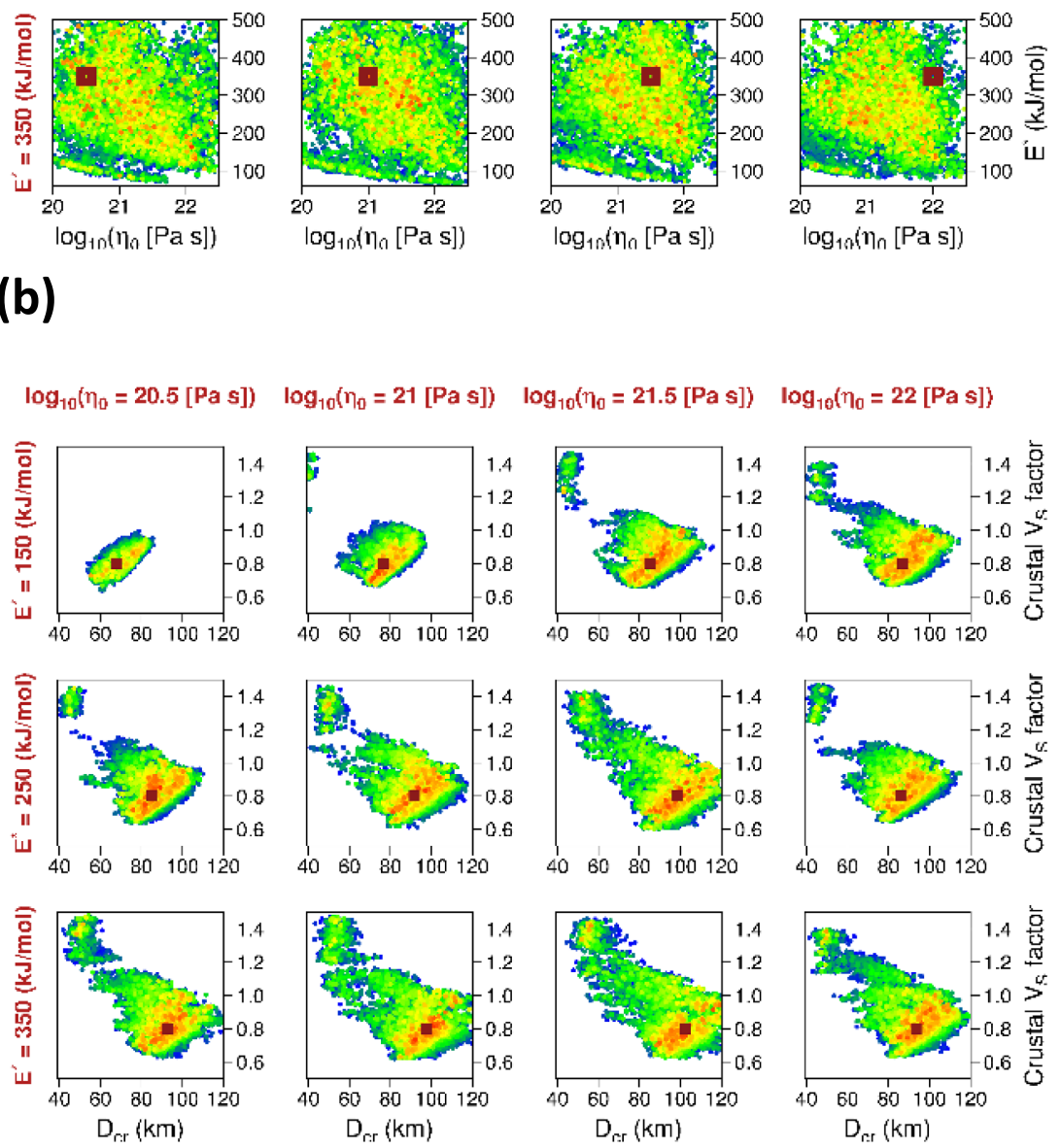

Figure 9. Inversion results using both body waves and surface waves arrival times, for 12 different input combinations of $\eta_{0}$ and $E^{*}$. The event locations (depth and epicentral distance) are inverted. (a) $\eta_{0}\left[\right.$ in $\left.\log _{10}(\mathrm{~Pa} \mathrm{~s})\right]$ as a function of $E^{*}$ (in kJ mol${ }^{-1}$ ), and (b) crustal thickness (in km) as a function of the $V_{S}$ factor in the crust, for all the models accepted by the Bayesian algorithm (coloured dots). Red and blue colours are small and large misfit values, respectively. The dark red squares correspond to the values to retrieve. The $\eta_{0}$ and $E^{*}$ input values are explicitly written in dark red at the top and on the left of each figure, respectively.

Note that all these models can fit the observational data within error bounds, as shown in Fig. 12. The dispersion of the $t_{R}-t_{\mathrm{P}}$ distributions (Fig. 12b) reflects the spread of the $V_{S}$ distributions. Unsurprisingly, the mean standard deviation for $t_{R}-t_{\mathrm{P}}$ is smaller for the geodynamic approach, at each period considered.
The comparison between the two inversion methods shows that the geodynamically constrained approach can be extremely powerful if one has good prior constraints on the structure of Mars, by producing consistent velocity models through the whole planet, even at depths where the seismic data are no more sensitive to the structure. However, as noted by Drilleau et al. (2020), these models will not be representative of Mars if the prior assumptions on 


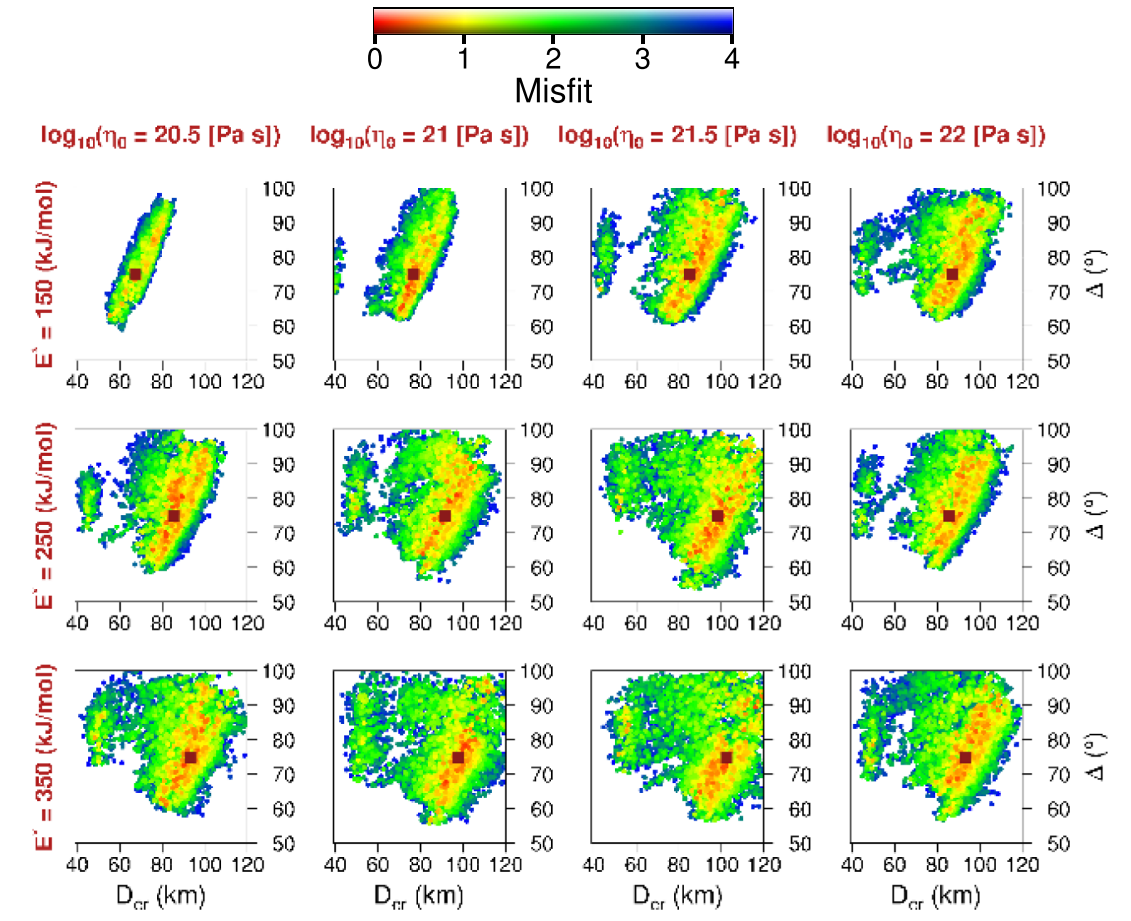

Figure 10. Inversion results using both body waves and surface waves arrival times, for 12 different input combinations of $\eta_{0}$ and $E^{*}$. The event locations (depth and epicentral distance) are inverted. The figures display the crustal thickness (in km) as a function of the epicentral distance $\Delta$ (in ${ }^{\circ}$ ), for all the models accepted by the Bayesian algorithm (coloured dots). Red and blue colours are small and large misfit values, respectively. The dark red squares correspond to the values to retrieve. The $\eta_{0}$ and $E^{*}$ input values are explicitly written in dark red at the top and on the left of each figure, respectively.

the physics turn out to be erroneous. On the other hand, the classical method can only provide constraints on the seismic profile at the depths where the data are sensitive, but can also allow for models that are inconsistent with a priori physical considerations, if the physics of Mars deep interior significantly differs from our expectations. For instance, Drilleau et al. (2020) shows an example of inversion results using a very challenging input model, with a temperature profile in the crust and mantle close to the liquidus. The results demonstrate that if the thermal properties of Mars are strongly anomalous, given our knowledge of Mars, the geophysical and/or geodynamic a priori assumptions could experience difficulties for fitting the data, contrary to the classical parametrization that benefits from a larger flexibility.

This suggests the importance and the usefulness of considering these complementary approaches when interpreting Mars seismic data.

\subsection{Inversions using body waves only}

In this section we investigate the ability of geodynamically constrained inversions to resolve the structure using a limited data set (a few events), composed exclusively of body waves. Indeed, up to now and despite the very low noise (Lognonné et al. 2020), most seismic event waveforms recorded by SEIS do not exhibit clear phase arrivals due to scattering, and no surface waves have yet been identified (Giardini et al. 2020; Lognonné et al. 2020). However, two large events near the Cerberus Fossae system (25-30 distance) with clear direct $P$ and $S$ wave identification have been recorded. For single station analysis, the absence of surface waves is making the estimation of both the quake location and the velocity structure more challenging. As demonstrated by Drilleau et al. (2020), constraining the velocity structure using body waves arrival times only is difficult, in the sense that the inversion will almost always find a combination of $V_{S}$ profiles and epicentral distance compatible with the data. However, in the framework of the InSight mission, impacts could be located by one of several orbiting cameras (Daubar et al. 2018), which could provide a known location, compared to marsquakes that have much less well-constrained locations. This will enable the direct inversion of all differential travel times with respect to $P$ arrival times.

\subsubsection{Localized impact events}

We first consider a pessimistic scenario, where we investigate the extent to which the crust and mantle structure could be constrained using two impact events. We then consider a more optimistic scenario, by testing inversions using several combinations of three and four events. We performed several inversions considering impacts occurring at different epicentral distances $(\Delta): 1.68^{\circ}, 8.4^{\circ}$, $33.7^{\circ}$ and $75^{\circ}$ (corresponding to a distance of 100, 500, 2000 and $4447 \mathrm{~km}$ along great circles). For each seismic ray path, the first $P$ and $S$-waves arrivals are considered. The reading error attributed to the arrival time estimates is $1 \mathrm{~s}$. As demonstrated in Daubar et al. (2018), the sensitivity of the data to the structure in depth is located near the turning point of the ray paths. These depths are approximately $5,70,150$ and $1100 \mathrm{~km}$, for $\Delta=1.68^{\circ}, 8.4^{\circ}, 33.7^{\circ}$ and $75^{\circ}$, respectively. Note that these values can vary according to the velocity model considered. The true geodynamic parameters are $\eta_{0}$ $=10^{21} \mathrm{~Pa} \mathrm{~s}$ and $E^{*}=150 \mathrm{~kJ} \mathrm{~mol}^{-1}$.

The results using two events are shown in Fig. 13. Only the combinations including the closest impact $\left(\Delta=1.68^{\circ}\right)$ are shown, the other ones providing no significant constraint on $\eta_{0}$ and $E^{*}$. We observe that $\eta_{0}$ and $E^{*}$ are not constrained when the closest event is combined with the two farthest events (Figs $13 \mathrm{a} 2$ and a3). When 


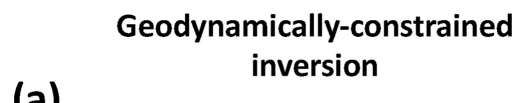

(a)

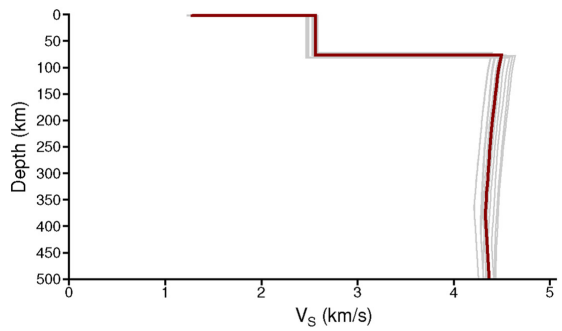

(c)
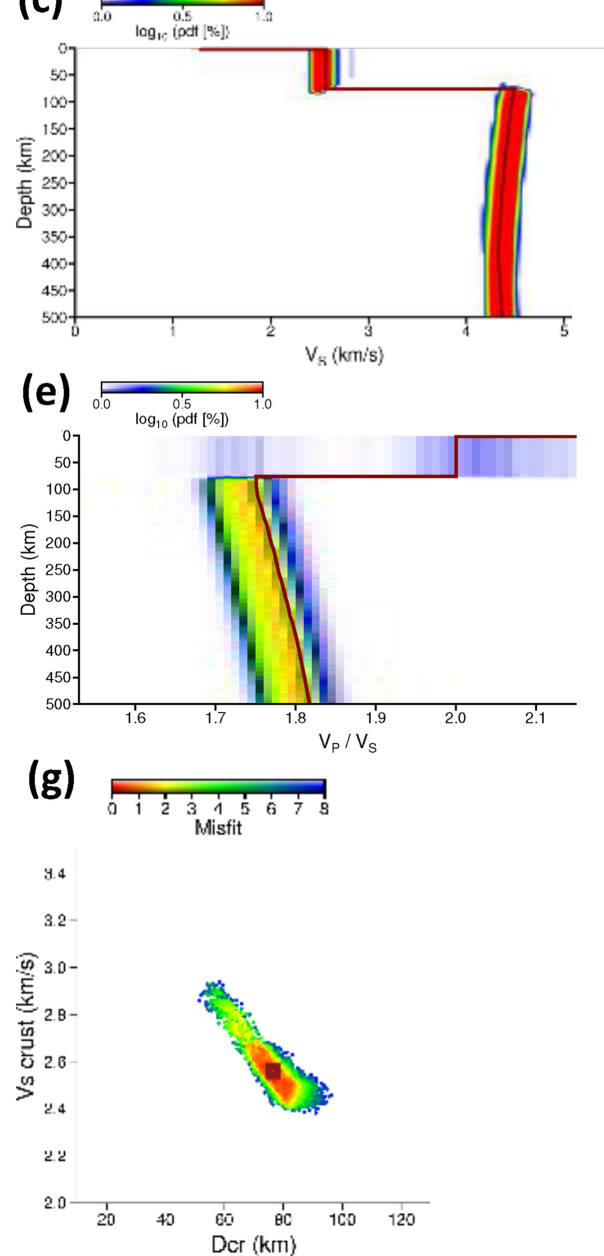

Classical

inversion

(b)

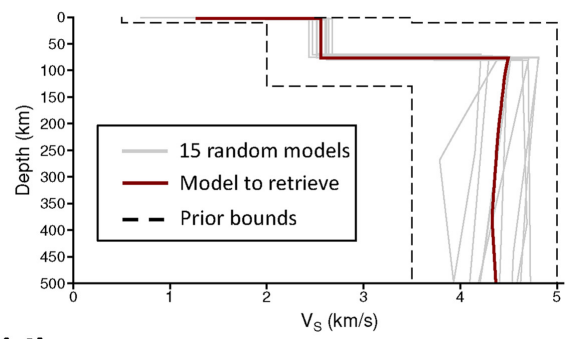

(d)

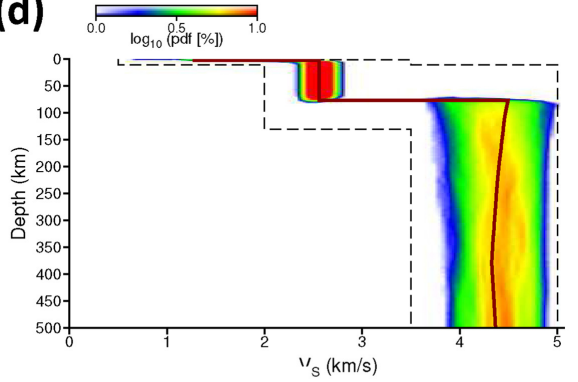

(f)

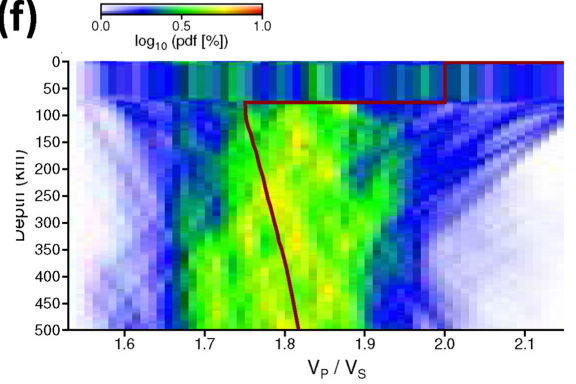

(h)
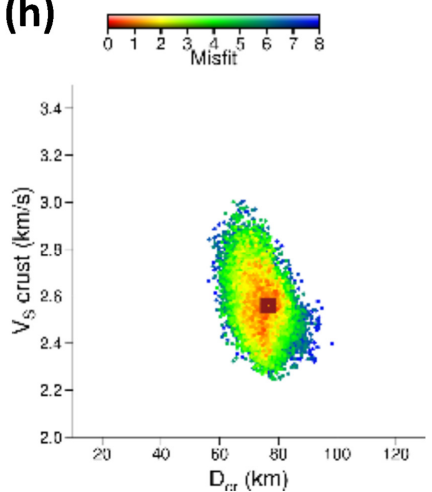

Figure 11. Inversion results using the geodynamic approach (left-hand panel) and the classical approach (right-hand panel), using body waves and surface waves as input. Panels (a) and (b) show in grey a random subset of 15 models selected from the ensemble solution. The dark red line is the $V_{S}$ profile to retrieve, obtained using $\eta_{0}=10^{21} \mathrm{~Pa} \mathrm{~s}$ and $E^{*}=150 \mathrm{~kJ} \mathrm{~mol}^{-1}$. The black dashed lines in (b) are the prior bounds. Panels (c) and (d) are colour density plots in logarithmic scale of 1-D $V_{S}$ profiles. Red and blue colours show high and low probabilities, respectively. The pdf values are computed by counting the number of profiles in each $0.05 \mathrm{~km} \mathrm{~s}^{-1} V_{S}$ interval every $1 \mathrm{~km}$ depth. For a given depth, the sum of the pdf over all the $V_{S}$ intervals is equal to 100 per cent. Panels (e) and (f) are colour density plots in logarithmic scale of the $V_{P} / V_{S}$ ratio. Panels (g) and (h) display the crustal thickness (in km) as a function of $V_{S}$ in the crust (in $\mathrm{km} \mathrm{s}^{-1}$ ), for all the models accepted by the Bayesian algorithm. Red and blue colours indicate small and large and high misfit values, respectively. The dark red squares correspond to the values to retrieve.

the data from the two closest events are used $\left(\Delta=1.68^{\circ}\right.$ and $8.4^{\circ}$, Fig. 13 a1), although very diffuse, we observe the appearance of a first branch of low misfit values, for $E^{*}<300 \mathrm{~kJ} \mathrm{~mol}^{-1}$. This is mainly explained by the fact that the seismic ray paths for these events are located in the crust. A better knowledge of the crustal velocity allows to decrease the trade-off between the crustal velocity and the crustal thickness, compared to the other combinations of impact events (Figs 13b1-b3). The seismic waves for the $33.7^{\circ}$ and $75^{\circ}$ events travel down to approximately 150 and $1100 \mathrm{~km}$ depth, respectively, and are consequently mainly sensitive to the mantle structure. They allow to reduce the range of possible $V_{S}$ values in the mantle, in particular with the $75^{\circ}$ event (Figs $13 \mathrm{c} 1-\mathrm{c} 3$ and Figs 13d1-d3), but the crustal thickness is unconstrained. 
(a) Body waves
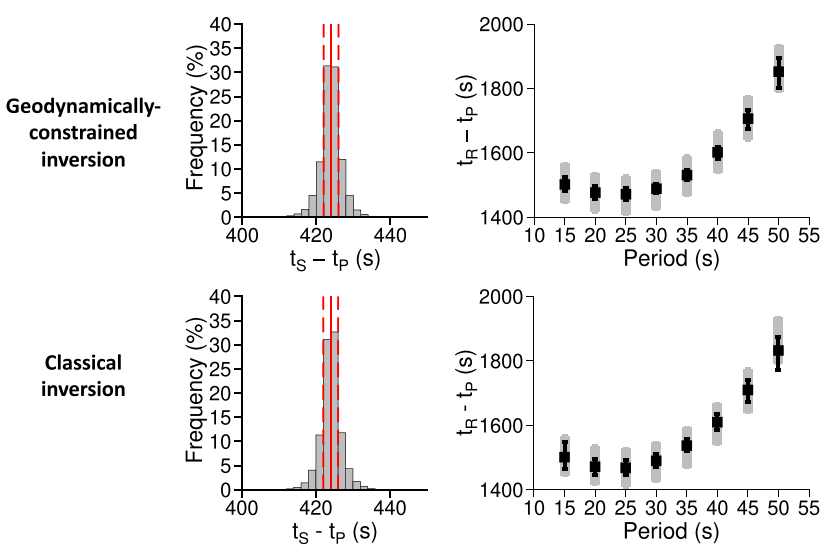

Figure 12. Datafit from the inversion of body waves and surface waves, considering a single event, for the geodynamic inversion (top panel) and the classical inversion (bottom). (a) Shows the output marginal probabilities of $t_{\mathrm{S}}-t_{\mathrm{P}}$. The red lines and dashed lines correspond to the input arrival times and uncertainties. (b) Mean value and \pm mean absolute deviation of $t_{R}-$ $t_{\mathrm{P}}$ as a function of period shown in black, for all sampled models. The thick grey lines show the uncertainty on the input data.

The importance of using arrival times belonging to an impact close to the station (for which the seismic waves are confined within the crust) is reinforced when considering three events in the inversion process (Fig. 14). The combination of the three farthest events $\left(\Delta=8.4+33.7+75^{\circ}\right)$ only allows to exclude $\eta_{0}$ larger than $10^{22} \mathrm{~Pa} \mathrm{~s}$ (Fig. 14a3). The crustal thickness is not well retrieved (Figs 14b3 and c3). However, when using the nearest event located at $1.68^{\circ}$ (Figs 1 a1 and a2), the areas of low misfit values are significantly reduced. The trade-off between $V_{S}$ in the crust and the crustal thickness is better reduced when considering the two closest events (Figs 14b1-b3 and 14c1-c3). These results imply that impacts events are required to be located at small, intermediate and large epicentral distances in order to use them to infer meaningful inferences about geodynamic parameters and crustal thickness, because their seismic ray paths convey information about the structure of Mars at different depths. One should remark that the maximum value of the epicentral distance required for the closest event depends on the true crustal thickness value along the ray path (thinner crust requires smaller close-by epicentral distances).

The results that rely on four events, located at $1.68^{\circ}, 8.4^{\circ}, 33.7^{\circ}$ and $75^{\circ}$, are shown in Fig. 15. The inversions were performed for 12 different pairs $\left(\eta_{0}, E^{*}\right)$. For intermediate true values of $E^{*}$ and $\eta_{0}$ (i.e. couples for which $\eta_{0}=10^{21}-10^{21.5} \mathrm{~Pa} \mathrm{~s}$, and $E^{*}=250$ and $350 \mathrm{~kJ} \mathrm{~mol}^{-1}$ ), the estimation of $E^{*}$ and $\eta_{0}$ is difficult. Compared to the results using both body waves and surface waves (Fig. 6a), the distributions of $E^{*}$ as a function of $\eta_{0}$ are different (Fig. 15a). For the cases where the true value for $\eta_{0}$ is $10^{22} \mathrm{~Pa} \mathrm{~s}, \eta_{0}$ is better constrained than when using both body waves and surface waves from a single event. Similar to the inversions using both surface waves and body waves, a range of possible $E^{*}$ values can be provided when the true $E^{*}$ is equal to $150 \mathrm{~kJ} \mathrm{~mol}^{-1}$, and when $\eta_{0}$ is lower than $10^{22} \mathrm{~Pa} \mathrm{~s}$, except that the appearance of a secondary branch is not observed here.

This difference between the results of the two types of inversion and the presence/absence of a secondary branch, are due to the transformation of olivine into wadsleyite, which creates a seismic discontinuity at depths between 900 and $1200 \mathrm{~km}$, depending on the model (see Fig. 2). Fig. 16(a) displays the marginal probabilities for $V_{S}$ at $1050 \mathrm{~km}$ depth for the joint body waves and surface waves inversion (in blue), and for pure body waves inversion using the four impact events (in pink). The true values of the geodynamic parameters to retrieve are $\eta_{0}=10^{21} \mathrm{~Pa} \mathrm{~s}, E^{*}=150 \mathrm{~kJ} \mathrm{~mol}^{-1}$. An interesting feature is that the marginal distribution of the body waves inversion is unimodal, with its maximum probability corresponding to the true $V_{S}$ value, whereas the marginal distribution of the joint body waves and surface waves inversion for a single event shows two maxima. This bimodal distribution means that the marginal distribution is influenced by the velocity values above and below the seismic discontinuity, and confirms that the surface waves are not able to constrain the structure at this depth, for the period range considered. On the contrary, a dataset composed exclusively of body waves with an event far enough $\left(\Delta=75^{\circ}\right)$ to yield sensitivity in the deeper part of the mantle, allows the velocity structure to be retrieved, and decreases the number of likely couples $\left(\eta_{0}, E^{*}\right)$, hence the trade offs between $\eta_{0}$ and $E^{*}$. Indeed, Figs 16(b) and (c) demonstrates that the two maxima of the marginal distribution for the joint the body waves and surface waves inversion correspond to the two branches of low misfit values for $\eta_{0}=10^{21} \mathrm{~Pa} \mathrm{~s}$ and $E^{*}=$ $150 \mathrm{~kJ} \mathrm{~mol}^{-1}$ displayed in Fig. 6(a). The $\left(\eta_{0}, E^{*}\right)$ couples for which $\eta_{0}<10^{21.1} \mathrm{~Pa} \mathrm{~s}$, produce $V_{S}$ values at $1050 \mathrm{~km}$ depth close to the true pair of values (Fig. 16b), whereas the $\left(\eta_{0}, E^{*}\right)$ couples with 200 $<E^{*}<500 \mathrm{~kJ} \mathrm{~mol}^{-1}$, and $10^{21.1}<\eta_{0}<10^{22.5} \mathrm{~Pa} \mathrm{~s}$, exhibit lower $V_{S}$ values (Fig. 16c), indicating that olivine has not undergone its transition to wadsleyite at this depth.

When using body waves only, the crustal thickness distributions are close to the true values (Fig. 15b), but the $V_{S}$ crustal factor is less constrained compared to the case where surface waves are used in the inversion (Fig. 7). These results can be explained by the distinct modes of propagation of the different waves. Indeed, surface waves are particularly sensitive to the region of the planet located between the surface and the upper mantle (for the period range considered), along the source-station path, whereas body waves have a sensitivity around the seismic ray path. Consequently, surface waves allow a better estimation of the crustal seismic velocities (in the case where only few events are used for the body waves inversions).

Our results demonstrate the advantage of using recorded impacts events distributed among different epicentral distances, in order to sample different part of the planet in depth, instead of detecting impacts located within the same epicentral distance range. In Fig. 17, we introduced random noise in the data using uncertainty bounds of 1, 2, $3 \mathrm{~s}$. Unsurprisingly, compared to the results without noise (Fig. 17a) the models accepted by the algorithm are more spread within the parameter space. By introducing random noise within \pm 3 per cent uncertainty, we observe the appearance of a second branch for $\eta_{0}=10^{22} \mathrm{~Pa} \mathrm{~s}$ and $E^{*}=350 \mathrm{~kJ} \mathrm{~mol}^{-1}$ (Fig. 17d). In other words, the noise introduce bias in the estimation of the reference viscosity.

\subsubsection{Comparison between the classical and the geodynamically constrained approaches}

The comparison between the classical and the geodynamically constrained models, using $P$ - and $S$-waves arrival times from the four impact events, are displayed in Fig. 18. As for the comparison using both body waves and surface waves (Section 3.1.3), the true $V_{S}$ profile was computed using $\eta_{0}=10^{21} \mathrm{~Pa} \mathrm{~s}$ and $E^{*}=150 \mathrm{~kJ} \mathrm{~mol}^{-1}$.

As noted in the previous section, we observe that $V_{S}$ in the crust is not well-retrieved, either for the geodynamically constrained models (Figs 18a, c and e) or for the classical models (Figs 18b, d and f), due to the absence of surface waves. 


\section{Inversions of $t_{p}, t_{s}$, using different combinations of $\mathbf{2}$ impact events}
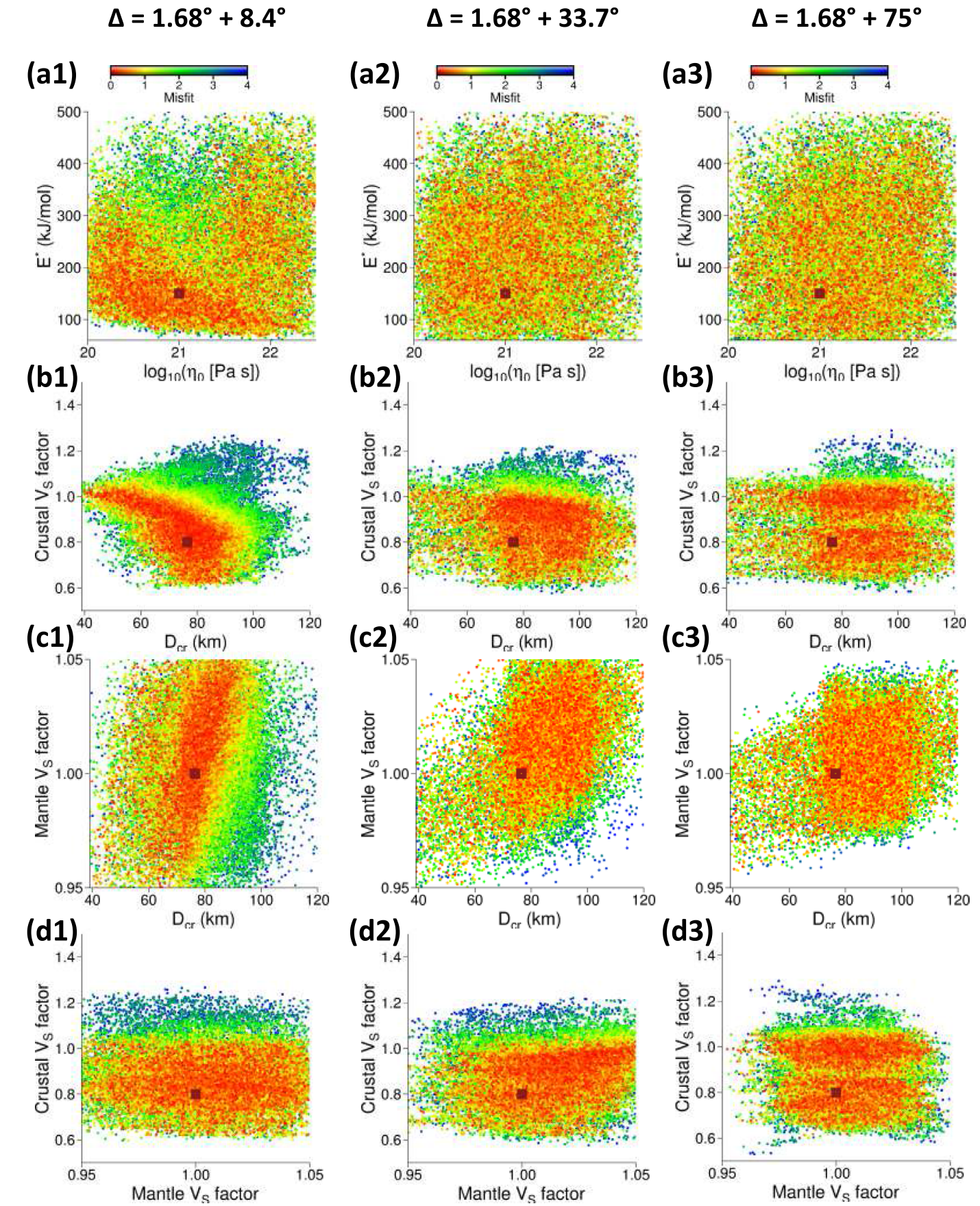

Figure 13. Inversion results using body waves arrival times, considering a combination of 2 impact events located at different epicentral distances. The figures show $\eta_{0}\left[\right.$ in $\left.\log _{10}(\mathrm{~Pa} \mathrm{~s})\right]$ as a function of $E^{*}\left(\right.$ in $\mathrm{kJ} \mathrm{mol}^{-1}$ ), and the crustal thickness (in km) as a function of the $V_{S}$ factor in the crust, for all the models accepted by the Bayesian algorithm (coloured dots). Red and blue colours are small and large misfit values, respectively. The dark red squares correspond to the values to retrieve.

In the mantle, the geodynamically constrained $V_{S}$ models are very close to the true $V_{S}$ profile (Figs 18a and c), as it was the case for the combined body waves and surface waves inversion (Figs 18a and c). The $1 \sigma$ standard deviation of the pdf around the true model is equal to 2 per cent. The pdf of the $V_{S}$ for the classical models (Fig. 18d) shows a multimodal solution, as highlighted by the random subset of models (Fig. 18b). The parameterization of the classical models is very flexible, in the sense that, unlike the geodynamically constrained models, a decrease of $V_{S}$ in the upper mantle is not required. Consequently, different models with a decrease and/or an increase of $V_{S}$ as a function of depth, can equally fit the differential arrival times $t_{S}-t_{P}$. The non-unicity of the solution is also exacerbated by the large uncertainty of $V_{S}$ in the crust. All these models yield arrival times compatible with the true data, as demonstrated in Fig. 19. 


\section{Inversions of $t_{p}, t_{s}$, using different combinations of 3 impact events} $\Delta=1.68^{\circ}+8.4^{\circ}+33.7^{\circ}$$$
\Delta=8.4^{\circ}+33.7^{\circ}+75^{\circ}
$$
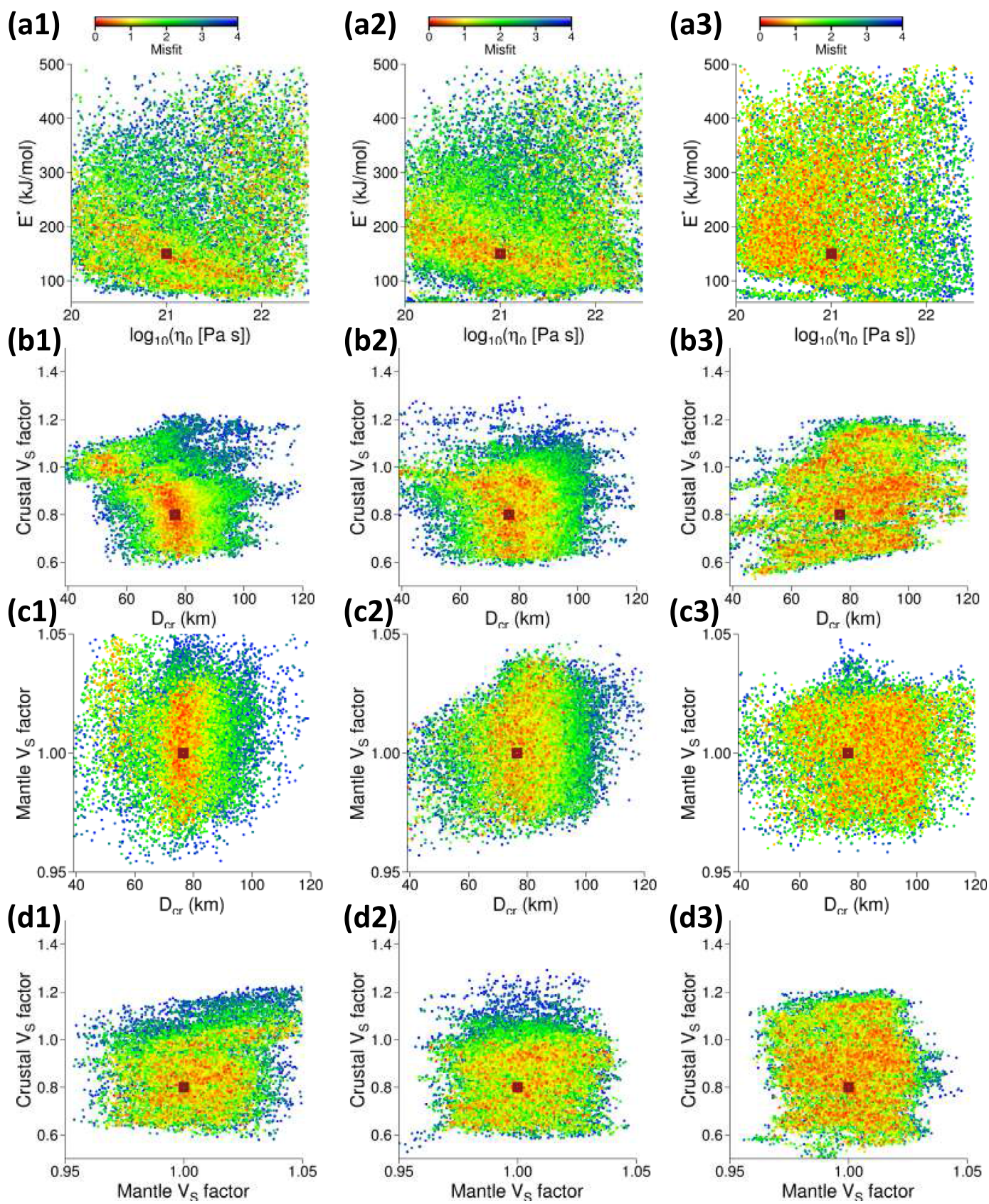

Figure 14. Inversion results using body waves arrival times, considering a combination of three impact events located at different epicentral distances. The figures show $\eta_{0}\left[\right.$ in $\left.\log _{10}(\mathrm{~Pa} \mathrm{~s})\right]$ as a function of $E^{*}\left(\right.$ in $\left.\mathrm{kJ} \mathrm{mol}^{-1}\right)$, and the crustal thickness (in km) as a function of the $V_{S}$ factor in the crust, for all the models accepted by the Bayesian algorithm (coloured dots). Red and blue colours are small and large misfit values, respectively. The dark red squares correspond to the values to retrieve.

The $V_{P} / V_{S}$ ratio in the mantle corresponds to the values to retrieve for the geodynamically constrained models (Fig. 18e), but the distribution is broader than in the case using both surface waves and body waves (Fig. 11e), due to the larger $V_{S}$ distribution in the crust. On the contrary, for the classical models, the range of the most probable $V_{P} / V_{S}$ values is smaller (Fig. 18f) compared to the results obtained with surface waves and body waves (Fig. 11f), because we are using here four $P$-waves arrival times, instead of only one when surface waves are considered.

Thanks to the a priori constraints in the mantle, the trade-offs between the seismic parameters are reduced, which allows a good estimation of the crustal thickness for the geodynamically constrained models (Fig. 18g). Without such tight constraints, a larger range of possible crustal thickness values is allowed for the classical models (Fig. 18h). In the absence of secondary waves arrivals, which are 
(a)
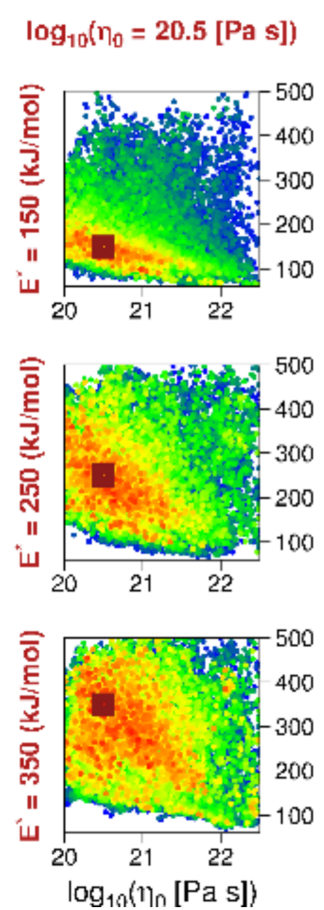

(b)
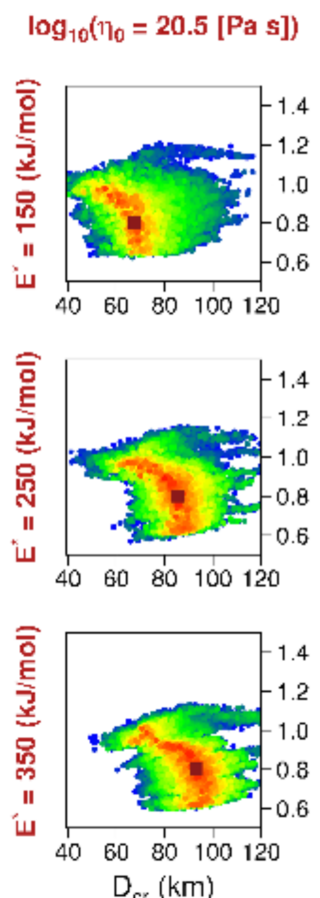

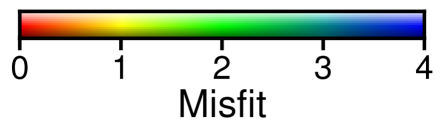

$\log _{10}\left(\eta_{0}=21[\mathrm{Pas}]\right) \quad \log _{10}\left(\eta_{0}=21.5[\mathrm{~Pa} s]\right) \quad \log _{10}\left(\eta_{0}=22[\mathrm{~Pa} s]\right)$
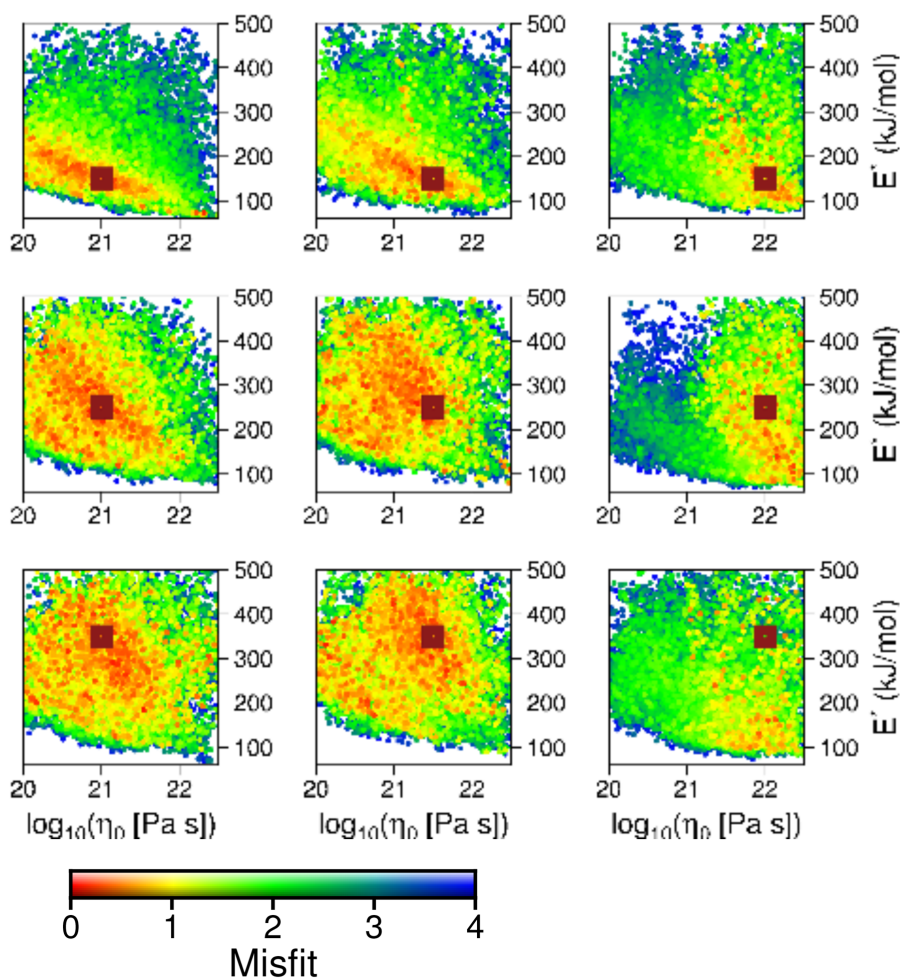

$\log _{10}\left(\eta_{\theta}=21\right.$ [Pa s]) $\log _{10}\left(\eta_{\theta}=21.5[\mathrm{~Pa} \mathrm{~s}]\right) \log _{10}\left(1_{0}=22[\mathrm{~Pa} \mathrm{~s}]\right)$
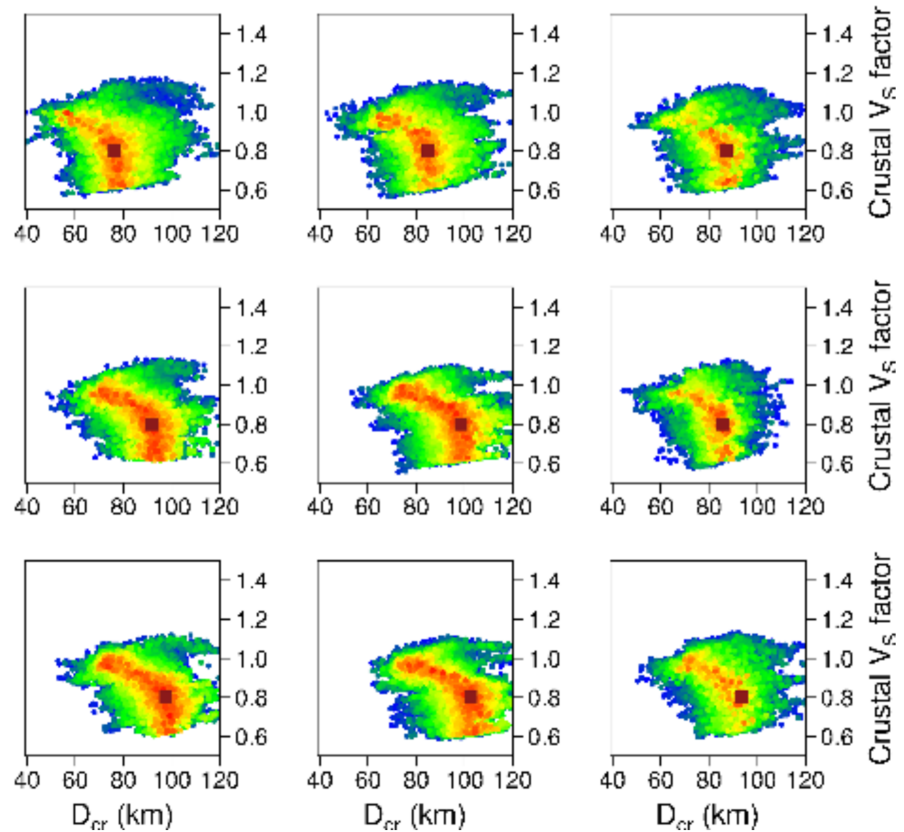

Figure 15. Inversion results using body waves arrival times, considering four events, for 12 different input combinations of $\eta_{0}$ and $E^{*}$. Panel (a) show $\eta_{0}$ [in $\left.\log _{10}(\mathrm{~Pa} \mathrm{~s})\right]$ as a function of $E^{*}\left(\right.$ in $\mathrm{kJ} \mathrm{mol}^{-1}$ ) and (b) the crustal thickness (in $\mathrm{km}$ ) as a function of the $V_{S}$ factor in the crust, for all the models accepted by the Bayesian algorithm (coloured dots). Red and blue colours are small and large misfit values, respectively. The dark red squares correspond to the values to retrieve. The $\eta_{0}$ and $E^{*}$ input values are explicitly written in dark red at the top and on the left of each figure, respectively. 
(a)

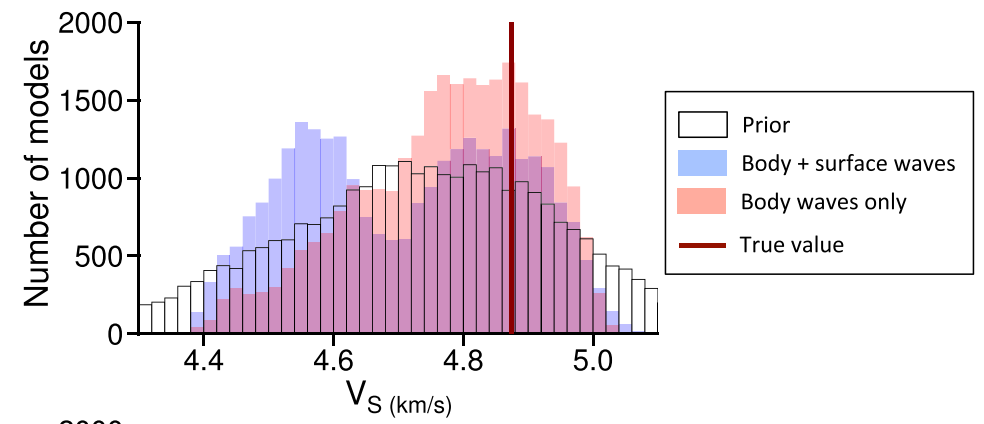

(b)

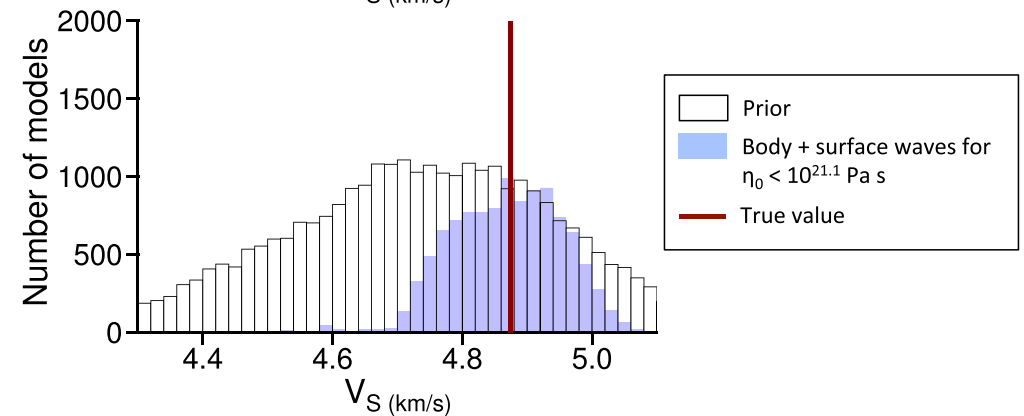

(c)

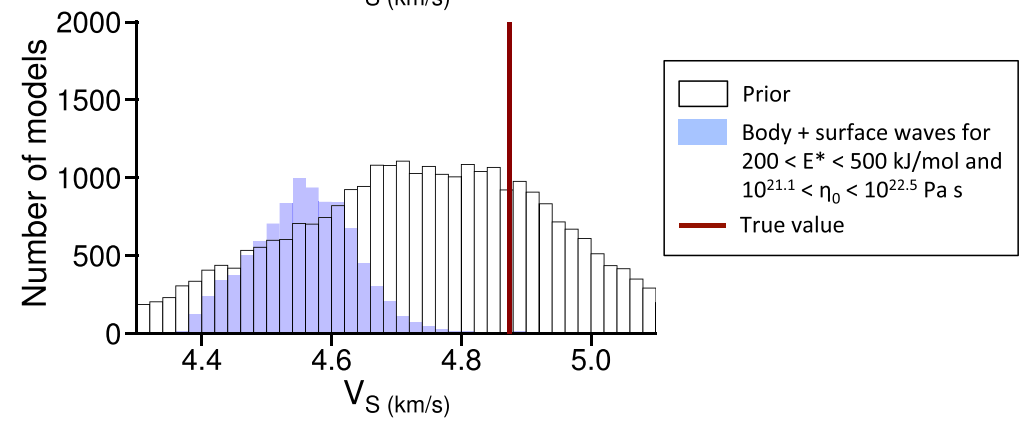

Figure 16. Marginal posterior distributions of $V_{S}$ at $1050 \mathrm{~km}$ depth (i.e. in the vicinity of the olivine-to-wadsleyite transition), for a single-event joint body waves and surface waves inversion (in blue), and for a four impact event pure body waves inversion (in pink). The marginal prior distribution is shown in black. The true values of the geodynamic parameters to retrieve are $\eta_{0}=10^{21} \mathrm{~Pa} \mathrm{~s}, E^{*}=150 \mathrm{~kJ} \mathrm{~mol}^{-1}$. The dark red line marks the true $V_{S}$ value at $1050 \mathrm{~km}$ depth. (a) Marginal distributions of all the models accepted by the algorithm. (b) Marginal distribution of body wave and surface wave inversion for all the accepted models for which $200<E *<500 \mathrm{~kJ} \mathrm{~mol}^{-1}$ and $10^{21.1}<\eta_{0}<10^{22.5} \mathrm{~Pa} \mathrm{~s}$. (c) Marginal distribution of body wave and surface wave inversion for all the accepted models for which $\eta_{0}<10^{21.1} \mathrm{~Pa}$ s.

very sensitive to sharp interfaces, only a smooth averaged profile can be estimated using the classical method.

The good agreement between synthetic data and inversion output shows here a clear potential of the both methods to resolve a first-order velocity structure of the Martian crust and mantle, using $P$ - and $S$-waves arrival times of impacts at known locations. The strong prior induced by the geodynamically constrained approach is powerful in the sense that this method avoids the ambiguity between several possible solutions, with the important caveat of explicitly assuming a 'known' composition and set of thermodynamic parameters for possible mineral assemblages. An ideal strategy with the InSight mission will be to iteratively improve the interior model as more data become available.

\section{DISCUSSION}

In this paper we showed how the 1-D structure of Mars could be investigated, using a limited seismic data set, with no more than one event large enough to record the first orbit of Rayleigh waves, or alternatively a small number of located impact events with body wave phases identified. With limited data, the modelling approach become crucial for understanding the significance of the resulting models.

We have shown that the geodynamic approach constitutes a powerful technique by allowing for tight constraints on the velocity structure from a relatively limited data set. In any Bayesian inversion, applying a tight prior reduces the size of the confidence intervals estimated from the posterior pdf. However, tight prior constraints also introduce a bias in the estimates computed from the sampled models. When inverting a data set consisting of body waves and surface waves, several events located at different epicentral distances may be required when applying a classical approach to obtain similar constraints on the final model distribution, compared to those based on a single event data set inverted using the geodynamic approach (Fig. 11). On the other hand, the geodynamically constrained approach induces by construction several assumptions. It assumes a known homogenous mantle composition, with mineralogical assemblages in thermodynamic equilibrium, and that the elastic and anelastic properties of those phase assemblages are well represented by the empirical relationships tuned to laboratory experiments and observations of Earth properties. The crustal thickness inferred from the geodynamic approach assumes on crustal production rates based on prescribed melting curves. The latter are not free 


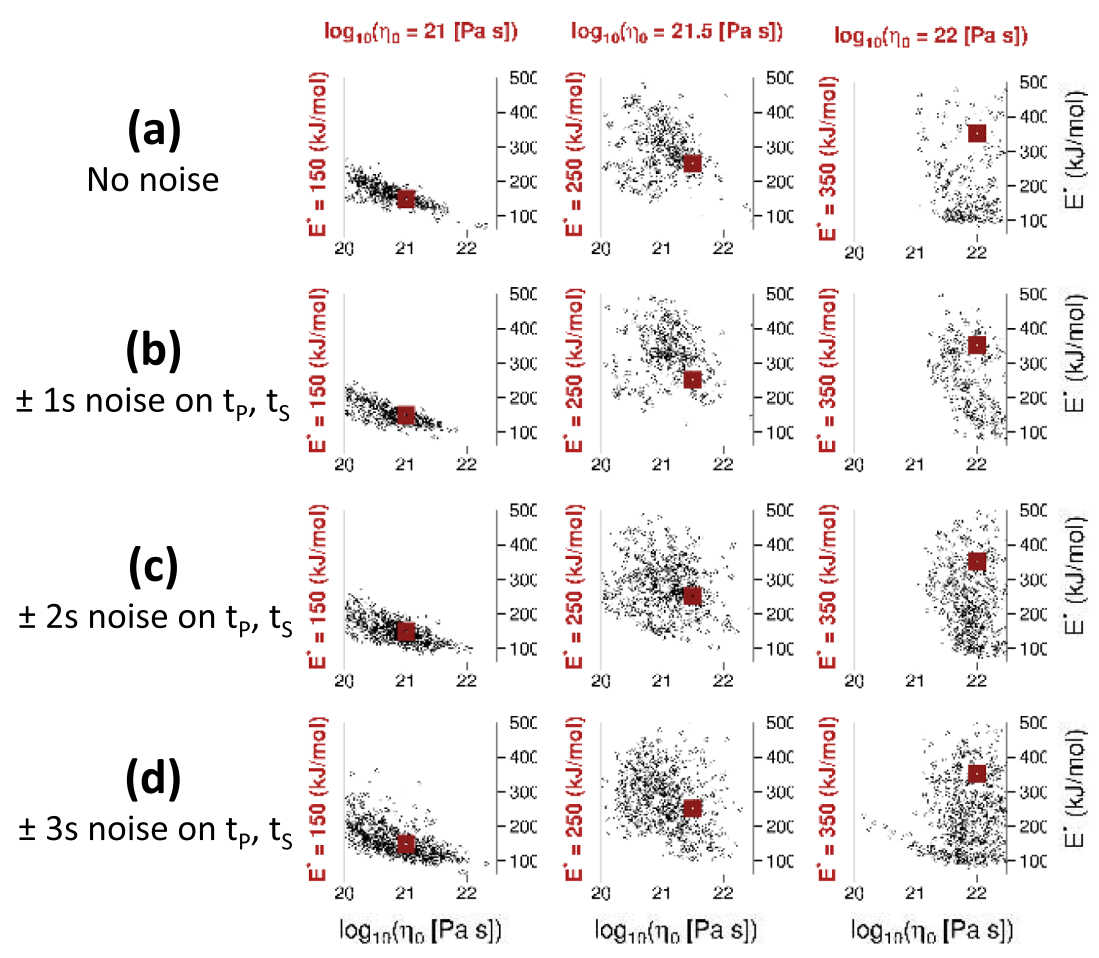

Figure 17. Inversion results using noisy body waves arrival times, considering 4 events, for three different input combinations of $\eta_{0}$ and $E^{*}$. The figures show $\eta_{0}[$ in $\log (\mathrm{Pa} \mathrm{s})]$ as a function of $E^{*}\left(\mathrm{in} \mathrm{kJ} \mathrm{mol}^{-1}\right)$ for the 10 percent models with the lowest misfit values. The dark red squares correspond to the values to retrieve. The $\eta_{0}$ and $E^{*}$ input values are explicitly written in dark red at the top and on the left of each figure, respectively. (a) Are the results with no noise. (b), (c) and (d) display the results considering 1,2 and 3 s noise on the $P$ and $S$ waves arrival times, and 3, 4 and 5 per cent on the surface waves arrival times, respectively.

of uncertainties and are composition dependent. It also considers that the temperature profiles are well represented by an evolving stagnant lid lithosphere, and an adiabatic convecting mantle layer throughout the entire planet history, while plate tectonics may have taken place during the first few hundreds of millions of years of Mars evolution (Nimmo \& Stevenson 2000; Breuer \& Spohn 2003).

We demonstrated the complementarity of using both inversions approaches parametrized with seismic velocities and geodynamic parameters, when handling such a non-linear problem. Obtaining similar results from inverting with and without geodynamic constraints will allow us to search for possible violations of the physical assumptions, or alternatively to verify that the assumptions are reasonable, allowing us to use the tighter constraints with confidence. In other words, the classical inversion can be useful to detect the possible inconsistencies in our prior assumptions.

\subsection{Potential implications for the thermochemical history of Mars}

As mentioned earlier, in addition to constrain the 1-D mantle rheological and seismological structure of the planet, the coupled seismic-geodynamic approach allows recovering the present-day thermochemical state of the planet, and to reconstruct its thermal history. Such ability is shown in Fig. 20, corresponding to the inversion displayed in Fig. 8(b) (for true values $\eta=10^{2} 1 \mathrm{~Pa}$ s and $E^{*}=$ $150 \mathrm{~kJ} \mathrm{~mol}^{-1}$ ) where the data set is assumed to contain surface waves and body waves. The 10 per cent best models (those associated with the lowest misfits) represent a small fraction of the prior, leading to well-constrained present-day mantle temperatures but a relatively wide plausible range for present-day core temperatures (Figs 20e and f). This leads to a relatively broad plausible range of areotherms that spans the colder side of the prior range (Fig. 20h). Similarly, the best models also moderately help constraining the present-day surface heat flux.

We performed the same exercise for the case where the data set is exclusively composed of body waves generated by four welllocalized impacts (Fig. 17b, $\eta=10^{21} \mathrm{~Pa} \mathrm{~s}$ and $E^{*}=150 \mathrm{~kJ} \mathrm{~mol}^{-1}$ ). The results are shown in Fig. 21 that also displays the models associated with the model output that have the 10 per cent lowest misfits. Interestingly, in this case, the present-day areotherm and associated heat flow are considerably better constrained (Figs $21 \mathrm{~g}$ and $h$ ).

As explained in Section 3.2.1, this result is closely related to the ability of the data set to constrain the seismic velocities at the olivine to wadsleyite phase change, which occurs near $1050 \mathrm{~km}$ depth for $\eta=10^{2} 1 \mathrm{~Pa} \mathrm{~s}$ and $E^{*}=150 \mathrm{~kJ} \mathrm{~mol}^{-1}$. The surface waves are not sensitive at this depth, whereas the four-event body waves data set we considered contains an impact event far enough to constrain the seismic velocities in the deep mantle. We showed in Fig. 15 that the most probable $\left(\eta_{0}, E^{*}\right)$ couples for the body waves inversion are aligned along a single branch, for true values equal to $\eta=10^{21} \mathrm{~Pa} \mathrm{~s}$ and $E^{*}=150 \mathrm{~kJ} \mathrm{~mol}^{-1}$, whereas for the inversions including surface waves (Fig. 6), a second branch appears between $200<E^{*}<500 \mathrm{~kJ}$ $\mathrm{mol}^{-1}$, and $10^{21.1}<\eta_{0}<10^{22.5} \mathrm{~Pa} \mathrm{~s}$. As shown in Fig. 16, values of the geodynamic parameters between $200<E^{*}<500 \mathrm{~kJ} \mathrm{~mol}^{-1}$, and $10^{21.1}<\eta_{0}<10^{22.5} \mathrm{~Pa} \mathrm{~s}$ have a tendency to shift the olivine to wadsleyite phase transformation to greater depths, compared to the true model. Such pairs of $\left(\eta_{0}, E^{*}\right)$ values are compatible with hottest present-day areotherm compared to the true ones, because the olivine to wadsleyite transformation has a positive Clapeyron 

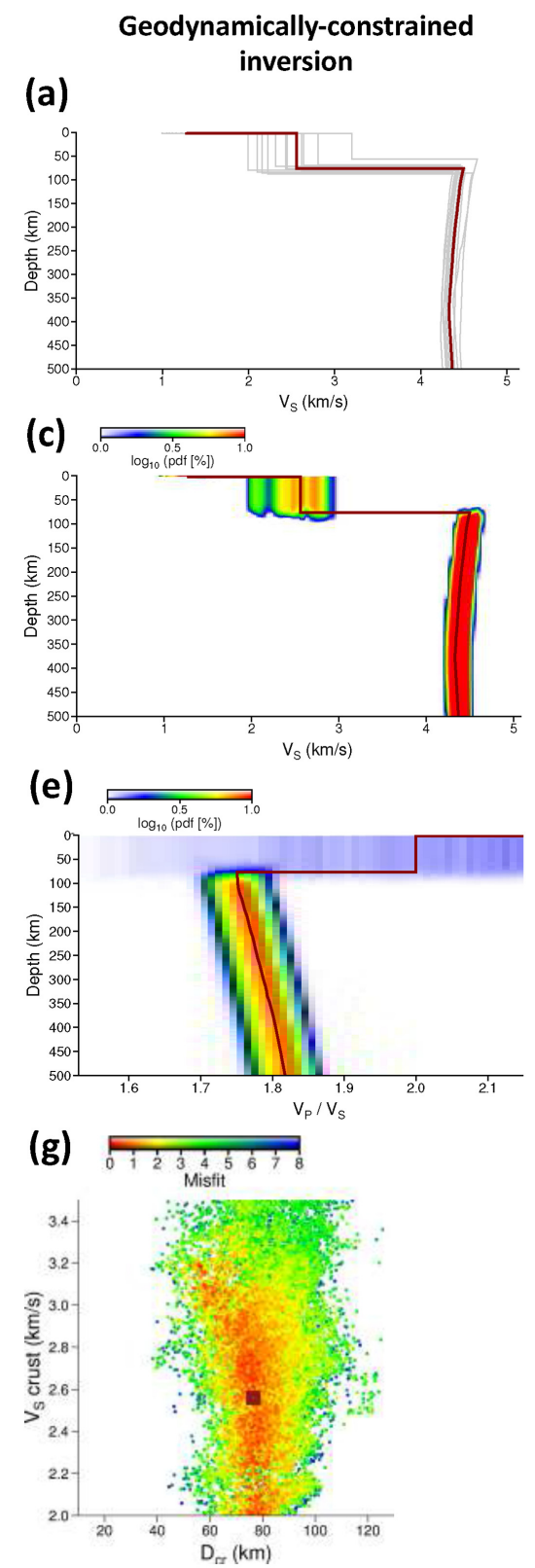

Classical

inversion

(b)
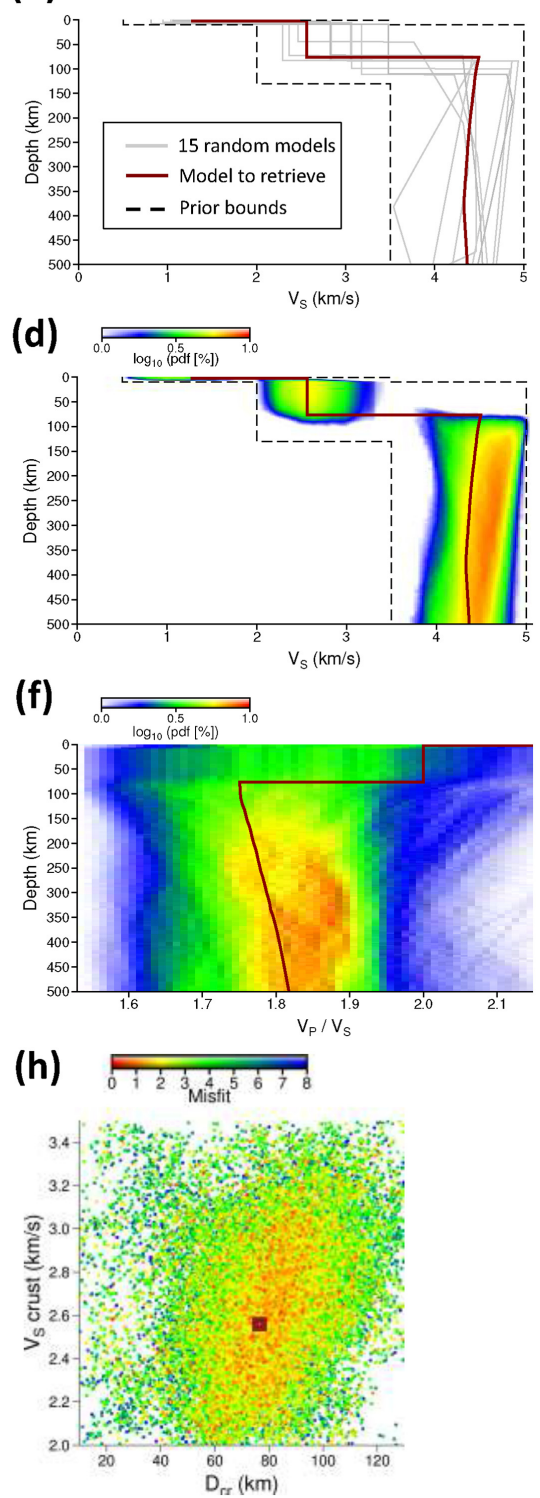

Figure 18. Inversion results using the geodynamic approach (left-hand panel) and the classical approach (right-hand panel), using $P$ - and $S$-wave arrival times from four events as input. Panels (a) and (b) show in grey a random subset of 15 models taken in the ensemble solution. The dark red line is the $V_{S}$ profile to retrieve, obtained using $\eta_{0}=10^{21} \mathrm{Pas}$ and $E^{*}=150 \mathrm{~kJ} \mathrm{~mol}^{-1}$. The black dashed lines in (b) are the prior bounds. Panels (c) and (d) are colour density plots in logarithmic scale of 1-D $V_{S}$ profiles in. Red and blue colours show high and low probabilities, respectively. The pdf values are computed by counting the number of profiles in each $0.05 \mathrm{~km} \mathrm{~s}^{-1} V_{S}$ interval at each depth. For a given depth, the sum of the pdf over all the $V_{S}$ intervals is equal to 100 per cent. Panels (e) and (f) are colour density plots in logarithmic scale of the $V_{P} / V_{S}$ ratio. Panels (g) and (h) display the crustal thickness (in km) as a function of $V_{S}$ in the crust (in $\mathrm{km} \mathrm{s}^{-1}$ ), for all the models accepted by the Bayesian algorithm. Red and blue colours indicate small and large misfit values, respectively. The dark red squares correspond to the values to retrieve.

slope (e.g. Akaogi et al. 2007). This explains why the present-day areotherm is better constrained using the dataset of four impact events compared to the single-event joint surface wave and body wave dataset.

\subsection{Influence of the mantle composition}

The true seismic data and the inversion results presented in Section 3 were obtained by assuming the mantle composition of Taylor (2013). To test the influence of the mantle composition on the inversion output with the same arrival times obtained with the mantle composition of Taylor (2013), we performed inversions using several other bulk mantle compositions. Assuming that the SNC meteorites are representative of the Martian crust, models based on geochemical arguments have been developed to infer the composition of the bulk silicate Mars [see Taylor (2013) for a review]. To derive the chemical composition of Mars from the chemical compositions of the Martian meteorites, two approaches are generally applied. The first approach uses correlations between elements in the Martian meteorites, assuming that refractory elements are 
(a) $\Delta=1.68^{\circ}$
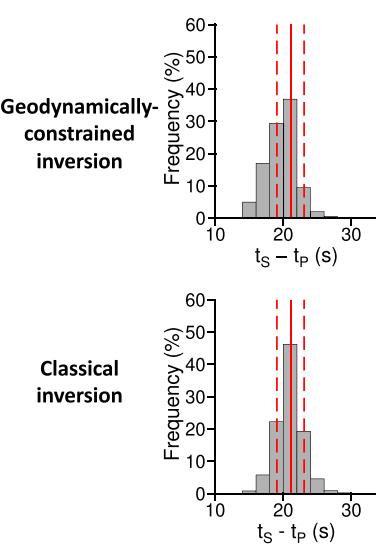

(b) $\Delta=8.4^{\circ}$
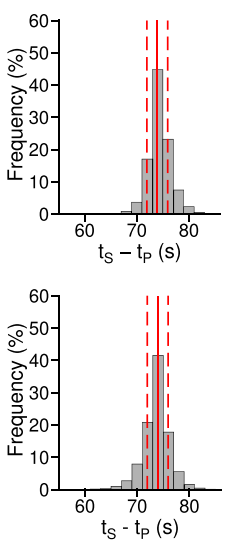

(c) $\Delta=33.7^{\circ}$
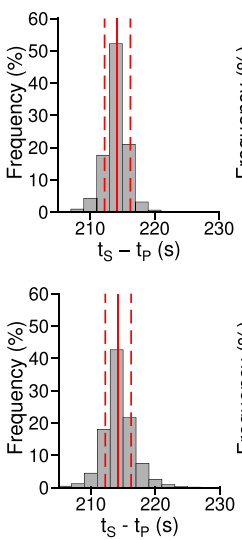

(d) $\Delta=75^{\circ}$
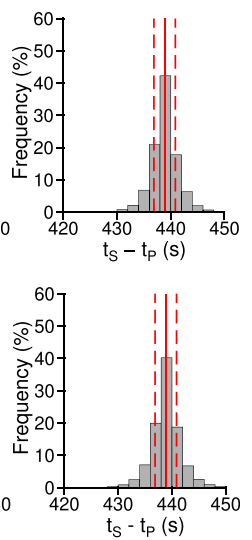

Figure 19. Datafit from the inversion of $P$ - and $S$-waves arrival times of four impact events. The figure shows the output marginal probabilities of $t_{\mathrm{S}}-t_{\mathrm{P}}$ from the geodynamic inversion (top panel) and the classical inversion (bottom panel). The red lines and dashed lines correspond to the input arrival times and uncertainties.
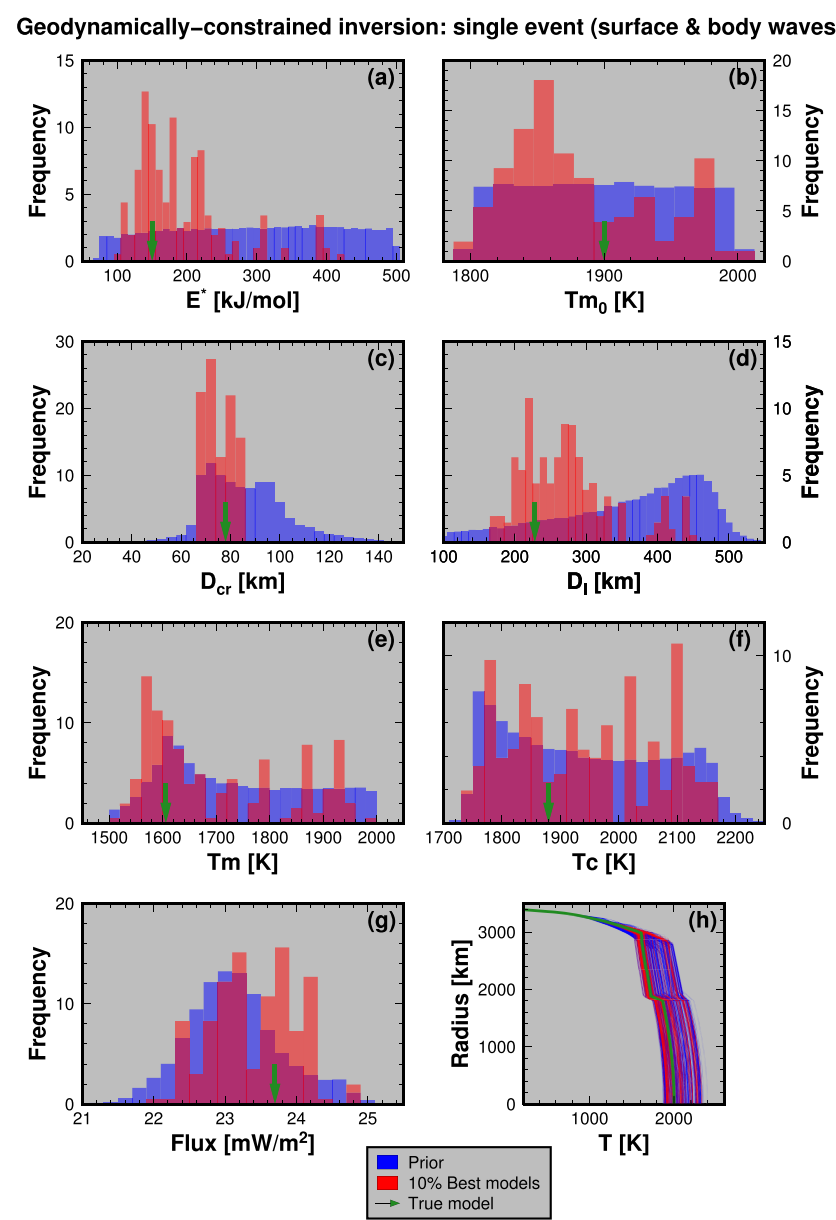

Figure 20. Illustration of the ability of the geodynamically constrained inversions to infer the present-day structure and to reconstruct the thermal history of the planet. Prior (blue) and posterior probability density functions associated with small misfit (red) corresponding to the inversion displayed in Fig. 8(b) for a data set composed of both surface waves and body waves data. The green arrows and lines indicate true model parameters (i.e. $T_{m_{0}}$ $=1900 \mathrm{~K}, \eta_{0}=10^{21} \mathrm{~Pa} \mathrm{~s}, E^{*}=150 \mathrm{~kJ} \mathrm{~mol}^{-1}$ ) and associated values (present-day temperatures and surface heat flux).
Geodynamically-constrained inversion: four events (body waves only)
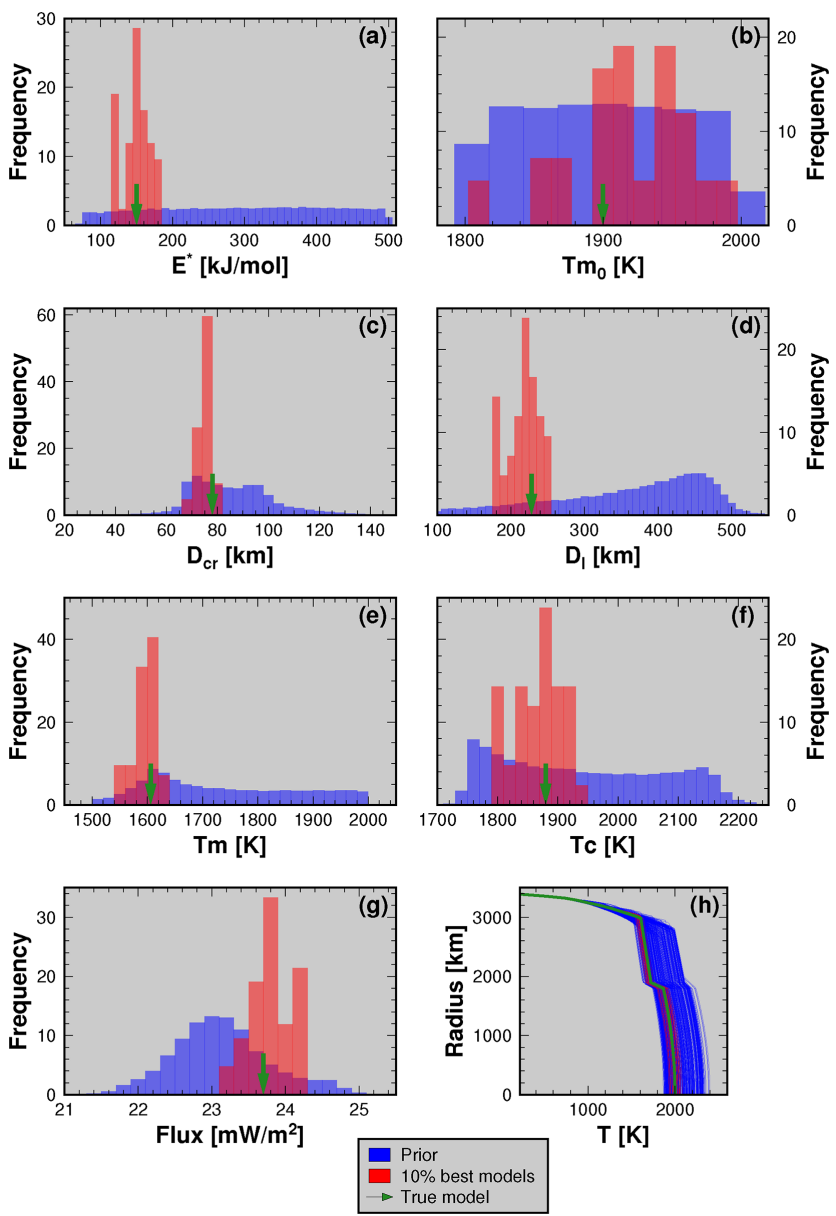

Figure 21. Same as Fig. 20 but for the case of a data set solely composed of body waves with well-localized sources, corresponding to the inversion displayed in Fig. 17(b) for that is $T_{m_{0}}=1900 \mathrm{~K}, \eta_{0}=10^{21} \mathrm{~Pa} \mathrm{~s}, E^{*}=$ $150 \mathrm{~kJ} \mathrm{~mol}^{-1}$.

present in chondritic proportions (Taylor 2013), while the second approach uses oxygen isotope systematics of the SNC meteorites 
and matches the elements abundances to mixtures of different chondritic material via mass balance equations (Lodders \& Fegley 1997; Sanloup et al. ; Mohapatra \& Murty 2003; Brasser et al. 2018).

We tested the compositions of Taylor (2013), Sanloup et al. (1999), Mohapatra \& Murty (2003), Lodders \& Fegley (1997) and Brasser et al. (2018). The results displayed in Fig. B1 of B show that modifying the bulk mantle composition does not significantly affect the results, which means that thermal variations and compositional layering have a more important effect than bulk compositional variations. Even though seismic velocity variations stem from both compositional and thermal states, it is not possible to decorrelate these two effects only on the basis of seismic data even when using our inversion approach that relies on additional thermal evolution constraints. However, one possibility to help discriminating among these compositional models would be to account for the inversion of the Heat-Producing Elements (HPE) content and their crust-mantle partitioning. Bulk HPE content varies in different mineralogical models, and crustal concentrations vary with thermal histories. This is an aspect of our inversion procedure that we could implement in the future, and which could be combined with heat flow measurements that the InSight $\mathrm{HP}^{3}$ experiment aims at performing at the landing site (Spohn et al. 2018).

In addition to seismic and geodetic data, electromagnetic induction data can be acquired at the surface of Mars by a magnetometer, such as the one on board of the InSight lander Johnson et al. (2020). The electromagnetic induction data can be used to infer a depth-dependent electrical conductivity profile (Banerdt et al. 2013), which is particularly sensitive to the mantle temperature and to its iron content. Therefore, combining electromagnetic induction data with seismic data can help reducing the trade-off between composition and temperature, and may significantly improve our knowledge on the mantle composition and temperature (e.g. Verhoeven et al. 2009).

\subsection{Influence of 3-D heterogeneities}

The seismic simulations and modelling discussed in this paper are based on spherically symmetric models. However, possible causes of seismic wavefield complexity such as 3-D structure and anisotropy, undeniably complexify the interpretation as a 1-D radial model considered here, and make the estimation of the geodynamic parameters more arduous. Based on seismological experience on Earth, the crust appears to have a very strong effect on seismic waves propagation, particularly on surface waves, which may be highly non-linear (e.g. Panning et al. 2010). On Earth, crustal thickness ranges from $\sim 7 \mathrm{~km}$ underneath oceans to $\sim 70 \mathrm{~km}$ underneath the deepest continents, and the average crustal thickness is about $24 \mathrm{~km}$. In contrast, on Mars, the crustal dichotomy between the Southern and Northern hemispheres suggested by gravity and topography measurements, indicates a crustal thickness varying from a few kilometers to more than $80 \mathrm{~km}$ (Zhong \& Zuber 2001; Neumann et al. 2004; Wieczorek \& Zuber 2004). Based on a spectral element modelling, Bozdağ et al. (2017) superimposed 3-D crustal thickness variations capturing the distinct crustal dichotomy between the Martian northern and southern hemispheres, as well as topography, and gravity to study the effect of the crust on body waves arrival times. Their simulations indicate that the 3-D crust modify the body waves arrival times compared to a reference 1-D model, whereas it significantly changes surface waveforms and their dispersive character depending on its thickness. While considering explicitly a thermochemical evolution with lateral variations would be computationally prohibitive, it is possible to include hemispheric crustal variations using our computationally efficient parametrized approach (Breuer et al. 1993; Thiriet et al. 2018). Considering these additional complexities will result in an increase the parameter space, and/or will require making additional a priori assumptions on the origin, and on the nature of lateral crustal variations.

\subsection{Effects of additional independent constraints on crustal thickness}

As discussed above, the crustal thickness exerts a dominant influence on the seismic inversion results, and modelling results can be used to constrain the prior probabilities that govern the Bayesian inversions. The crustal thickness and structure below the seismic station can also be determined using receiver functions (Panning et al. 2017; Drilleau et al. 2020), The local Moho thickness estimation provides a fundamental anchoring point for the determination of the crustal thickness variation of the whole planet from topography and gravity data. This approach has been successfully applied on the Moon where the Moho thickness at the Apollo 12/14 site has been combined with gravity and topography data obtained from the Clementine and Lunar Prospector missions to derive a crustal thickness map (Chenet et al. 2006).

Using receiver functions extracted from realistic synthetic seismograms as recorded by InSight, Drilleau et al. (2020) have shown that the Moho depth below the seismic station could by retrieved with an uncertainty of $\pm 10 \mathrm{~km}$. Therefore, we accounted for an additional constraint on the Moho depth in our inversion process, in order to evaluate the extent to which the trade-off between the geodynamic parameters could be reduced, by excluding models with a crustal thickness thinner or thicker than $\pm 2, \pm 5$ and $\pm 10 \mathrm{~km}$, compared to the true value. The results are displayed in Figs 22(a) and (b), considering both body waves and surface waves, and body waves only, respectively. It turns out that the reduction of the possible range of values for the geodynamic parameters $\left(\eta_{0}, E^{*}\right)$ seems to be difficult to achieve, even when using the crustal thickness input from receiver functions. Indeed, due to the highly nonlinear nature of the problem, the crustal thickness uncertainty needs to be very small to reduce the trade-off between $\eta_{0}$ and $E^{*}$. However, as mentioned above, the $\mathrm{HP}^{3}$ heat flow measurements could be used to define tighter prior constraints on the input thermal profile for the modelling including mineral physics constraints.

In Fig. $\mathrm{C} 1$ in $\mathrm{C}$, we show to what extent the trade-off between $V_{S}$ at the bottom of the crust and $V_{S}$ at the top of the mantle can be reduced, using prior knowledge deduced from receiver functions. The reduction of the trade-off is particularly visible when only body waves are used with the classical inversion, which means that a good knowledge of the crustal thickness will tighten the distribution of the seismic velocities. This effect is less noticeable when surface waves are considered, because of their sensitivity to the crust.

\section{CONCLUSIONS}

In the framework of the InSight mission, we anticipated in this study the recovery of seismic data capable of resolving the deep interior structure of Mars.

We tested probabilistic approaches to infer the deep structure of Mars in the frame of a single station-multiple events configuration motivated by the recent deployment of the first seismometer at the surface of Mars. This single-station setup necessarily yields a sparse, and uneven seismic data set that requires specific tailored 
(a) Geodynamically-constrained inversion (surface $\&$ body waves)

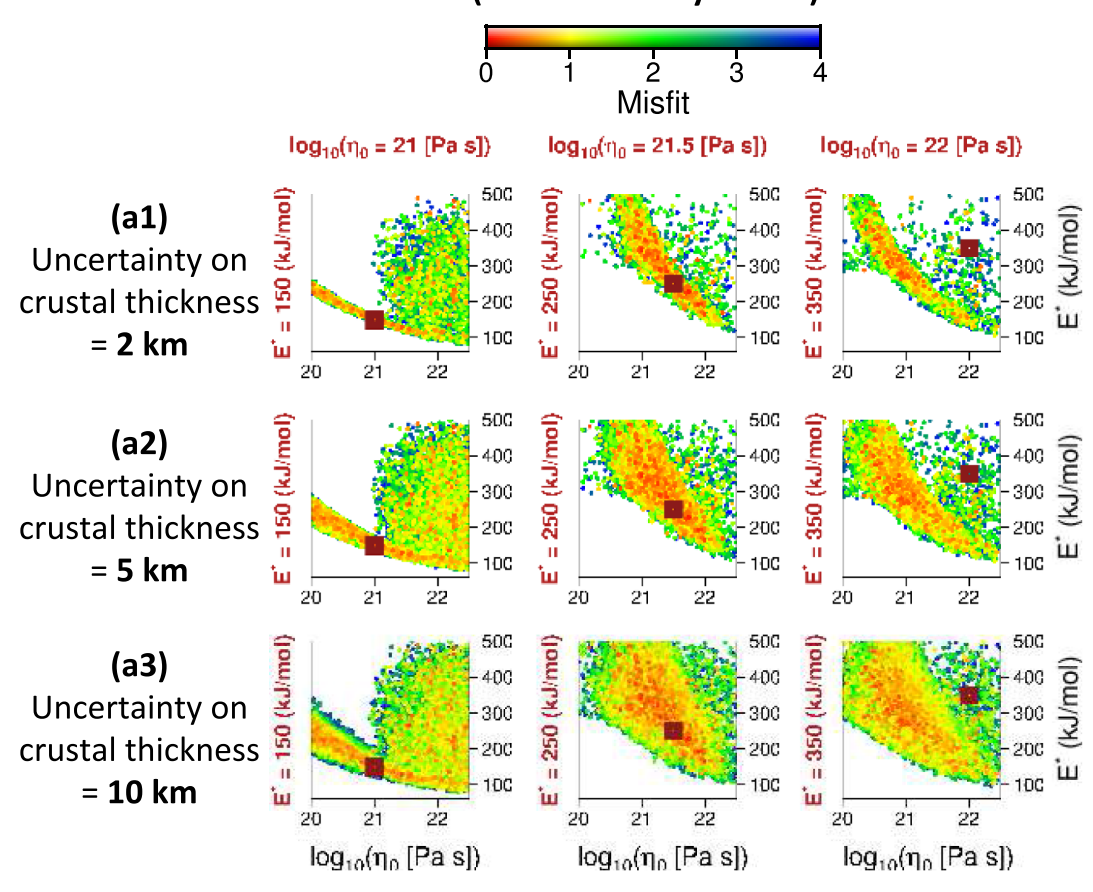

(b) Geodynamically-constrained inversion: four events (body waves only)

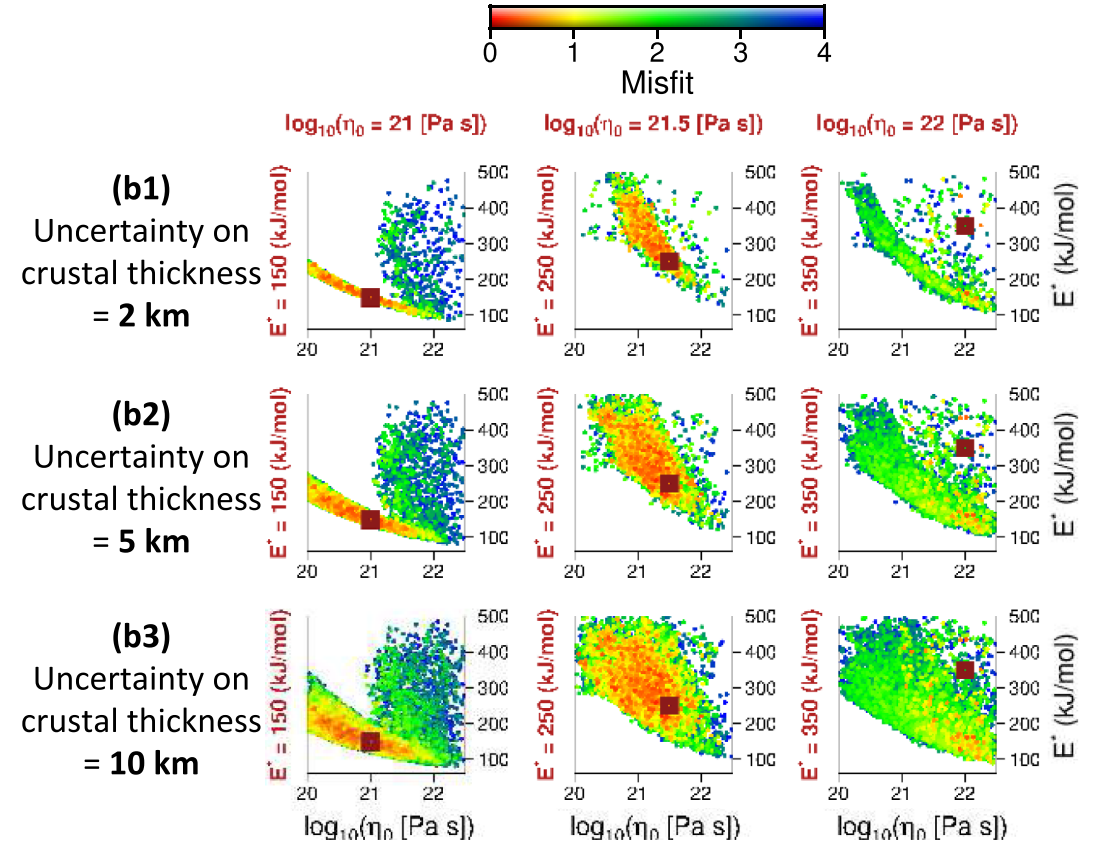

Figure 22. Inversion results using (a) both body waves and surface waves arrival times and (b) using body waves arrival times only, for three different input combinations of $\eta_{0}$ and $E^{*}$. Only the models for which crustal thickness is equal to that of the input value with an uncertainty of $\pm 2 \mathrm{~km}$ (a1, b1), $\pm 5 \mathrm{~km}$ (a2, b2) and $\pm 10 \mathrm{~km}(\mathrm{a} 2, \mathrm{~b} 3)$ are accepted by the algorithm. The figures show $\eta_{0}\left[\log _{10}(\mathrm{~Pa} \mathrm{~s})\right]$ as a function of $E^{*}$ (in $\mathrm{kJ}$ mol ${ }^{-1}$ ), for all the models accepted by the Bayesian algorithm (coloured dots). Red and blue colours indicate small and large misfit values, respectively. The dark red squares correspond to the values to retrieve. The input values for $\eta_{0}$ and $E^{*}$ are explicitly written in dark red at the top and on the left of each figure, respectively.

approaches to make the best use of it. We considered different types of plausible hypothetical data sets depending on the presence or the absence of surface waves, body waves events of internal or external origin, with or without constrained locations of the seismic events, different epicentral distances, different number or events and different noise levels. 
In order to infer the 1-D seismic structure of the crust and upper mantle, we compared two distinct approaches based on McMC. We analysed both their performances and their associated limitations as a function of the type of Martian seismic data set. One approach is a geodynamically constrained inversion that relies on a parametrization that incorporates the long-term thermochemical evolution of the planet. The second approach relies on a more classical Bézier curves parametrization of the 1-D seismic velocity profiles.

Using both body waves and surface waves from a single seismic event, we demonstrated the ability of the geodynamically constrained inversions to increase the prior knowledge on the model parameters meaningfully. The relationship between the model misfit and the parameter space is primarily governed by the crustal thickness, which strongly and non-linearly depends on the values of the parameters that govern the evolution of the planet: the mantle rheology and the initial thermal state of the planet. The shape of these areas becomes more complex with increasing true values of mantle reference viscosity and activation energy, due to the nonlinearity of the forward problem. The crustal thickness and shear wave crustal velocities are reasonably well retrieved.

In absence of surface waves, the knowledge of the event location is mandatory to constrain the internal structure. We investigated the case of one to four meteoroid impacts for which we assumed that the location at the surface of Mars has been determined from pictures taken by orbiting cameras (Daubar et al. 2018). Inversions of body waves arrival times from the impact sources taken individually are not able to constrain the geodynamic parameters or the seismic velocity profile. However, we showed that using several impact events, including at least one located at close epicentral distance, to sample independently the crust and the mantle, can provide constraints on the geodynamic parameters and on the interior structure. The maximum value of the epicentral distance required for the closest event depends on the true crustal thickness value along the ray path. Using impact events only, the retrieved crustal seismic velocities and crustal thickness show larger uncertainties compared to the results using both body waves and surface waves. These uncertainties could be reduced for a larger number of impact sources. In the frame of the InSight project, the mission has recently been extended for almost another Martian year (i.e. until the end of 2022). If this additional time window does not allow one to record and to identify impact events close enough to the station to illuminate the crust only, further constraints from receiver functions or reflected waves $(P P$, $P P P, S S, S S S$ ) could help reducing the ranges of possible seismic velocities values in the crust and of crustal thicknesses values.

Both inversion approaches tested have complementary advantages and limitations. The prior on the seismic structure induced by the geodynamically constrained inversion is significantly more informative than the prior used in the classical method, allowing to efficiently retrieve the seismic structure, and can also constrain the values important but poorly known governing parameters (mantle rheology, initial thermal state) along with the entire planetary thermochemical history. However, the approach can fail to converge towards the true solution, if the implied physical and thermodynamic assumptions tied to the geodynamic and mineralogical forward problems happen to be irrelevant to Mars. The classical inversion exhibits a generally more limited resolving power, but is far less affected by a priori assumptions because is uses a considerably less informative prior. For these reasons, exploiting the complementarity between these two approaches could be beneficial to constrain the present-day structure and the evolution of Mars, even with a very sparse and scarce seismic data set, and to assess the robustness of the inversion output. As a matter of fact, while surface waves have not yet been clearly identified with InSight, the geodynamically constrained approach may provide fundamental clues into the present-day state and the history of Mars, and may also be suitable for forthcoming seismic exploration of other terrestrial bodies.

\section{ACKNOWLEDGEMENTS}

This work was granted access to the HPC resources of CINES under the allocation A0070407341, made by GENCI. We thank Boris Dintrans, director of CINES, for his efficient handling of our request for computational time. A. R. was financially supported by the Belgian PRODEX program managed by the European Space Agency in collaboration with the Belgian Federal Science Policy Office. This is InSight Contribution Number 133. We thank the Editor, an anonymous reviewer, and Boris Kaus for their thoughtful comments that improved the manuscript.

\section{REFERENCES}

Acuña, M.H. et al., 1998. Magnetic field and plasma observations at Mars: initial results of the Mars global surveyor mission, Science, 279, 16761680 .

Akaogi, M., Takayama, H., Kijitani, H., Kawaji, H. \& Atake, T., 2007. Low-temperature heat capacities, entropies and enthalpies of $\mathrm{Mg}_{2} \mathrm{SiO}_{4}$ polymorphs, and $\alpha-\beta-\gamma$ and post-spinel phase relations at high pressure, Phys. Chem. Miner. 34(3), 169-183.

Banerdt, W.B. et al., 2013. InSight: a discovery mission to explore the interior of Mars, in Proceedings of the Lunar and Planetary Science Conference, 1915.

Banerdt, W.B. et al., 2020. Initial results from the InSight mission on Mars, Nat. Geosci., 13, 183-189.

Baratoux, D., Samuel, H., Michaut, C., Toplis, M.J., Monnereau, M., Wieczorek, M., Garcia, R. \& Kurita, K., 2014. Petrological constraints on the density of the martian crust, J. geophys. Res., 119(7), 1707-1727.

Blake, D.F. et al., 2013. Curiosity at Gale Crater, Mars: characterization and analysis of the rocknest sand shadow, Science, 341(6153), 1-7.

Boynton, W.V. et al., 2007. Concentration of H, Si, Cl, K, Fe, and Th in the low- and mid-latitude regions of Mars, J. geophys. Res., 112(12), 1-15, doi:10.1029/2007JE002887.

Bozdağ, E. et al., 2017. Simulations of seismic wave propagation on mars, Space Sci. Rev., 211(1), 571-594.

Brasser, R., Dauphas, N. \& Mojzsis, S.J., 2018. Jupiter's influence on the building blocks of Mars and Earth, Geophys. Res. Lett., 45(12), 59085917.

Breuer, D. \& Spohn, T., 2003. Early plate tectonics versus single-plate tectonics on Mars: evidence from magnetic field history and crust evolution, J. geophys. Res., 108, doi:10.1029/2002JE001999.

Breuer, D., Spohn, T. \& Wüllner, U., 1993. Mantle differentiation and the crustal dichotomy of Mars, Planet. Space Sci., 41(4), 269-283.

Chenet, H., Lognonné, P., Wieczorek, M. \& Mizutani, H., 2006. Lateral variations of lunar crustal thickness from the Apollo seismic data set, Earth planet. Sci. Lett., 243(1), 1-14.

Christensen, U.R., 1983. Convection in a variable-viscosity fluid: Newtonian versus power-law rheology, Earth planet. Sci. Lett., 64, 153-162.

Connerney, J.E.P., Acuña, M.H., Ness, N.F., Spohn, T. \& Schubert, G., 2004. Mars crustal magnetism, Solar Syst. Res., 11, 1-32.

Connolly, J.A.D., 2005. Computation of phase equilibria by linear programming: a tool for geodynamic modeling and its application to subduction zone decarbonation, Earth planet. Sci. Lett., 236, 524-541.

Daubar, I. et al., 2018. Impact-seismic investigations of the InSight mission, Space Sci. Rev., 214, doi:10.1007/s11214-018-0562-x.

Drilleau, M., Beucler, E., Mocquet, A., Verhoeven, O., Moebs, G., Burgos, G., Montagner, J.-P. \& Vacher, P., 2013. A Bayesian approach to infer radial models of temperature and anisotropy in the transition zone from surface wave dispersion curves, Geophys. J. Int., 195(2), 1165-1183. 
Drilleau, M. et al., 2020. MSS/1: single-station and single-event Marsquake inversion, Earth Space Sci., 7(12), doi:10.1029/2020EA001118.

Folkner, W.M., Yoder, C.F., Yuan, D.N., Standish, E.M. \& Preston, R.A., 1997. Interior structure and seasonal mass redistribution of mars from radio tracking of mars pathfinder, Science, 278(5344), 1749-1752.

Gagnepain-Beyneix, J., Lognonné, P., Chenet, H., Lombardi, D. \& Spohn, T., 2006. A seismic model of the lunar mantle and constraints on temperature and mineralogy, Phys. Earth planet. Inter., 159(3), 140-166.

Garcia, R.F., Gagnepain-Beyneix, J., Chevrot, S. \& Lognonné, P., 2011. Very preliminary reference moon model, Phys. Earth planet. Inter., 188(1), 96113.

Garcia, R.F. et al., 2019. Lunar seismology: an update on interior structure models, Space Sci. Rev., 215(8), 50.

Giardini, D. et al., 2020. The seismicity of mars, Nat. Geosci., 13(3), 205212.

Hahn, B.C., McLennan, S.M. \& Klein, E.C., 2011. Martian surface heat production and crustal heat flow from Mars Odyssey Gamma-Ray spectrometry, Geophys. Res. Lett., 38(14), 1-5.

Hartmann, W.K. et al., 1999. Evidence for recent volcanism on Mars from crater counts, Nature, 397, 586-589.

Hastings, W.K., 1970. Monte Carlo sampling methods using Markov chains and their applications, Biometrika, 57, 97-109.

InSight Mars SEIS Data Service, 2019. SEIS Raw Data: InSight Mission, IPGP, JPL, CNES, ETHZ, ICL, MPS, ISAE-Supaero, LPG, MSFC doi:10.18715/SEIS.INSIGHT.XB 2016.

InSight Marsquake Service, 2020. InSight Marsquake Service Mars Seismic Catalogue: InSight Mission V1 2/1/2020, ETHZ, IPGP, JPL, ICL, ISAESupaero, MPS, Univ. Bristol, doi:10.12686/a6.

Johnson, C.L. et al., 2020. Crustal and time-varying magnetic fields at the InSight landing site on Mars, Nat. Geosci., 13(3), 199-204.

Karato, S.-I. \& Wu, P., 1993. Rheology of the upper mantle: a synthesis, Science, 260, 771-778.

Khan, A. \& Mosegaard, K., 2002. An inquiry into the lunar interior: a nonlinear inversion of the Apollo lunar seismic data, J. geophys. Res., 107(E6), 3-1-3-23.

Khan, A., Mosegaard, K., Williams, J.G. \& Lognonné, P., 2004. Does the moon possess a molten core? Probing the deep lunar interior using results from LLR and lunar prospector, J. geophys. Res., 109(E9), doi:10.1029/2004JE002294.

Khan, A., Connolly, J.A.D., Pommier, A. \& Noir, J., 2014. Geophysical evidence for melt in the deep lunar interior and implications for lunar evolution, J. geophys. Res., 119(10), 2197-2221.

Khan, A. et al., 2016. Single-station and single-event marsquake location and inversion for structure using synthetic Martian waveforms, Phys. Earth planet. Inter, 258, 28-42.

Kiefer, W.S. \& Li, Q., 2016. Water undersaturated mantle plume volcanism on present-day Mars, Meteor. Planet. Sci., 51(11), 1993-2010.

Konopliv, A.S., Yoder, C.F., Standish, E.M., Yuan, D.-N. \& Sjogren, W.L., 2006. A global solution for the mars static and seasonal gravity, mars orientation, phobos and deimos masses, and mars ephemeris, Icarus, 182(1), 23-50.

Lodders, K. \& Fegley, B., 1997. An oxygen isotope model for the composition of Mars, Icarus, 126(2), 373-394.

Lognonné, P., Gagnepain-Beyneix, J. \& Chenet, H., 2003. A new seismic model of the moon: implications for structure, thermal evolution and formation of the moon, Earth planet. Sci. Lett., 211(1), 27-44.

Lognonné, P. \& Mosser, B., 1993. Planetary seismology, Surv. Geophys., 14(3), 239-302.

Lognonné, P. et al., 2019. Seis: InSight's seismic experiment for internal structure of mars, Space Sci. Rev., 215(1), 12.

Lognonné, P. et al., 2020. Constraints on the shallow elastic and anelastic structure of Mars from InSight seismic data, Nat. Geosci., 13(3), 213-220.

Masters, G., Woodhouse, J.H. \& Gilbert, F., 2011. Mineos v1.0.2 [software], Computational Infrastructure for Geodynamics. Retrieved from https://geodynamics.org/cig/software/mineos/.

Matsumoto, K., Yamada, R., Kikuchi, F., Kamata, S., Ishihara, Y., Iwata, T., Hanada, H. \& Sasaki, S., 2015. Internal structure of the Moon inferred from Apollo seismic data and selenodetic data from GRAIL and LLR, Geophys. Res. Lett., 42(18), 7351-7358.

McEwen, A.S. et al., 2007. Mars reconnaissance orbiter's high resolution imaging science experiment (HiRISE), J. geophys. Res., 112(5), 1-40.

Metropolis, N., Rosenbluth, A.W., Rosenbluth, M.N., Teller, A.H. \& Teller, E., 1953. Equation of state calculations by fast computing machines, $J$. Chem. Phys., 21, 1087-1091.

Mohapatra, R.K. \& Murty, S.V. S., 2003. Precursors of Mars: constraints from nitrogen and oxygen isotopic compositions of martian meteorites, Meteor. Planet. Sci., 38, 225-242.

Mosegaard, K. \& Tarantola, A., 1995. Monte-Carlo sampling of solutions to inverse problems, J. geophys. Res., 100, 12 431-12 447.

Nakamura, Y., 1983. Seismic velocity structure of the lunar mantle, J. geophys. Res., 88(B1), 677-686.

Neukum, G. et al., 2004. Recent and episodic volcanic and glacial activity on Mars revealed by the high resolution stereo camera, Nature, 432, 971979.

Neumann, G.A., Zuber, M.T., Wieczorek, M.A., McGovern, P.J., Lemoine, F. G. \& Smith, D.E., 2004. Crustal structure of mars from gravity and topography, J. geophys. Res., 109(E8), doi:10.1029/2004JE002262.

Nimmo, F. \& Stevenson, D.J., 2000. Influence of early plate tectonics on the thermal evolution and magnetic field of Mars, 105(E5), 11 969-11979.

Ohtani, E., Suzuki, A. \& Kato, T., 1998. Flotation of Olivine and Diamond in Mantle Melt at High Pressure: Implications for Fractionation in the Deep Mantle and Ultradeep Origin of Diamond, pp. 227-239, American Geophysical Union (AGU).

Panning, M.P., Lekić, V. \& Romanowicz, B.A., 2010. Importance of crustal corrections in the development of a new global model of radial anisotropy, J. geophys. Res., 115(B12), doi:10.1029/2010JB007520.

Panning, M.P., Beucler, E., Drilleau, M., Mocquet, A., Lognonné, P. \& Banerdt, W.B., 2015. Verifying single-station seismic approaches using earth-based data: preparation for data return from the insight mission to mars, Icarus, 248, 230-242.

Panning, M.P. et al., 2017. Planned products of the mars structure service for the insight mission to mars, Space Sci. Rev., 211(1), 611-650.

Plesa, A.-C., Tosi, N., Grott, M. \& Breuer, D., 2015. Thermal evolution and Urey ratio of Mars, J. geophys. Res., 120(5), 995-1010.

Rivoldini, A., Van Hoolst, T., Verhoeven, O., Mocquet, A. \& Dehant, V., 2011. Geodesy constraints on the interior structure and composition of Mars, Icarus, 213(2), 451-472.

Samuel, H., Lognonné, P.H., Panning, M. \& Lainey, V., 2019. The rheology and thermal history of mars revealed by the orbital evolution of Phobos, Nature, 569, 523-527.

Sanloup, C., Jambon, A. \& Gillet, P., 1999. A simple chondritic model of Mars, Phys. Earth. planet. Int., 112(1-2), 43-54.

Schubert, G., Cassen, P. \& Young, R.E., 1979. Subsolidus convective cooling histories of terrestrial planets, Icarus, 38, 192-211.

Schumacher, S. \& Breuer, D., 2006. Influence of a variable thermal conductivity on the thermochemical evolution of Mars, J. geophys. Res., 111, doi:10.1029/2005JE002429.

Shearer, P.M., 2009. Introduction to Seismology, 2nd edn, Cambridge Univ. Press.

Smith, D.E., Sjogren, W.L., Tylter, G.L., Balmino, G., Lemoine, F.G. \& Konopliv, A.S., 1999. The gravity field of Mars: results from Mars global surveyor, Science, 286(5437), 94-97.

Smrekar, S.E. et al., 2019. Pre-mission insights on the interior of mars, Space Sci. Rev., 215, doi:10.1007/s11214-018-0563-9.

Spohn, T. et al., 2018. The heat flow and physical properties package (HP $\left.{ }^{3}\right)$ for the InSight mission, Space Sci. Rev., 214(5), 96.

Stixrude, L. \& Lithgow-Bertelloni, C., 2005. Thermodynamics of mantle minerals. I. Physical properties, 162(2), 610-632.

Tarantola, A., 2005. Inverse Problem Theory and Methods for Model Parameter Estimation., SIAM.

Taylor, G.J., 2013. The bulk composition of Mars, Chemie der Erde - Geochemistry, 73(4), 401-420.

Thiriet, M., Michaut, C., Breuer, D. \& Plesa, A.-C., 2018. Hemispheric dichotomy in lithosphere thickness on Mars caused by differences in crustal structure and composition, J. geophys. Res., 123(4), 823-848. 
Toksoz, M.N., Dainty, A.M., Solomon, S.C. \& Anderson, K.R., 1974. Structure of the moon., Rev. Geophys. Space Phys., 12, 539-567.

Van Hoolst, T. \& Rivoldini, A., 2014. Interior structure and evolution of Mars, in Encyclopedia of the Solar System, 3rd edn, Chapter 18, pp. 379-396, eds Spohn, T., Breuer, D. \& Johnson, T.V., Elsevier.

Verhoeven, O. et al., 2009. Constraints on thermal state and composition of the Earth's lower mantle from electromagnetic impedances and seismic data, J. geophys. Res., 114, B03302.

Wieczorek, M.A. \& Zuber, M.T., 2004. Thickness of the Martian crust: improved constraints from geoid-to-topography ratios, J. geophys. Res., 109(1), 1-16.

Yoder, C.F., Konopliv, A.S., Yuan, D.N., Standish, E.M. \& Folkner, W.M., 2003. Fluid core size of mars from detection of the solar tide, Science, 300(5617), 299-303.

Zhong, S. \& Zuber, M.T., 2001. Degree-1 mantle convection and the crustal dichotomy on Mars, Earth planet. Sci. Lett., 189(1), 75-84.

Zuber, M., Smith, D., Solomon, S., Muhleman, D., Head, J., Garvin, J., Abshire, J. \& Bufton, J., 1992. The Mars Observer laser altimeter investigation, J. geophys. Res., 97(E5), 7781-7797.

\section{APPENDIX A: GEODYNAMICALLY CONSTRAINED INVERSION WITH EFFECTIVE ACTIVATION VOLUME AS A FREE PARAMETER}

We performed an inversion test for the geodynamic parametrization where we also invert for the effective activation volume $V^{*}$ in addition to the other parameters listed in Table 1 for the geodyamically constrained inversion. Although the experimental range for $V^{*}$ is rather large for silicate rocks $\left(V^{*}=0-25 \mathrm{~cm}^{3} \mathrm{~mol}^{-1}\right)$, thermalorbital constraints for Mars indicate that the plausible range for $V^{*}$ is more restricted: $V^{*}=0-4 \mathrm{~cm}^{3} \mathrm{~mol}^{-1}$ (Samuel et al. 2019). Here we considered a larger range: $V^{*}=0-7 \mathrm{~cm}^{3} \mathrm{~mol}^{-1}$ to allow for more flexibility. Values beyond this interval would not be compatible with thermal-orbital constraints, with the presence of an early dynamo on Mars and may not even allow for mantle convection, which is difficult to reconcile with the recent traces of volcanism on Mars (Hartmann et al. 1999; Neukum et al. 2004).

The results are displayed in Fig. A1 for a case analogous to that of Fig. 6 in which $V^{*}$ is fixed. The misfit shown in Fig. A1 remains very similar to the corresponding inversion where $V^{*}$ is fixed. This weak influence is due to the fact that within the Martian context, the limited plausible range for $V^{*}$ implies that this parameter does not strongly affect the inversion results, notably because a change in $V^{*}$ would lead to relatively modest changes in mantle viscosity in the vicinity of thermal boundary layers (in particular the upper one), compared to the ranges considered for other rheological parameters (e.g. $\left.\eta_{0}\right)$.

\section{APPENDIX B: EFFECT OF THE MINERALOGICAL COMPOSITION}

Fig. B1 shows the results of the geodynamically constrained inversion, considering the mineralogical compositions of Taylor (2013)
(TA), Sanloup et al. (1999) (EH), Mohapatra \& Murty (2003) (MM), Lodders \& Fegley (1997) (LF) and Brasser et al. (2018) (BR). We clearly observe that considering different bulk mantle compositions does not significantly affect the results, using both surface waves and body waves, or body waves only.

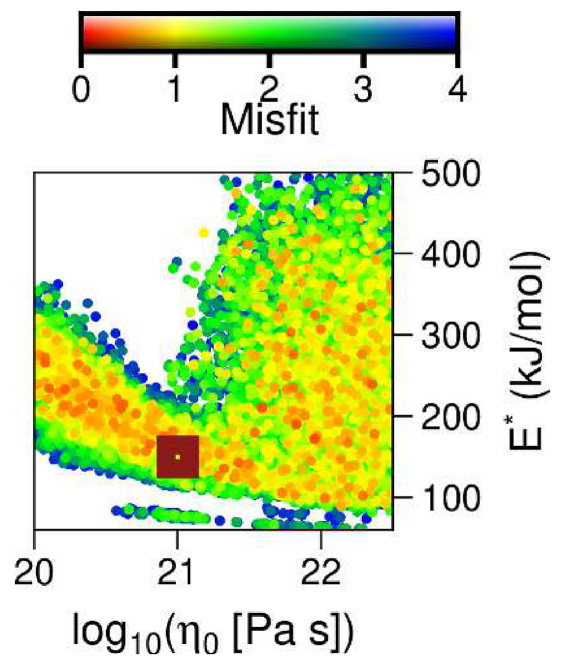

Figure A1. Inversion results using both body waves and surface waves arrival times, considering that $V^{*}$ can vary between 0 and $7 \mathrm{~cm}^{3} \mathrm{~mol}^{-1}$. The figure shows $\eta_{0}$ as a function of $E^{*}$, for all the models accepted by the Bayesian algorithm (coloured dots). Red and blue colours indicate small and large misfit values, respectively. The dark red square corresponds to the values to retrieve $\left(\eta_{0}=10^{21} \mathrm{~Pa} \mathrm{~s}\right.$ and $\left.E^{*}=150 \mathrm{~kJ} \mathrm{~mol}^{-1}\right)$.

\section{APPENDIX C: EFFECT OF ADDITIONAL CONSTRAINTS FROM RECEIVER FUNCTIONS}

Receiver functions can provide additional information on the the crustal thickness below the seismic station. To investigate the extent to which the seismic models could be improved using such a constraint, we show in Fig. C1 how $V_{S}$ at the top of the mantle varies as a function of $V_{S}$ at the bottom of the crust. The results are displayed for both the geodynamically constrained and the classical inversions, considering a priori uncertainties of $\pm 2, \pm 5$ and $\pm 10 \mathrm{~km}$ around the true value of the crustal thickness. Due to the inherent tight constraints applied on the geodynamically constrained approach compared to the classical parametrization, the effect of adding a supplementary constraint on the crustal thickness is more visible on the results of the classical inversion (Fig. C1b). However, this effect is less noticeable when surface waves are considered (Fig. C1b1) compared to the case with body waves only (Fig. C1b2), because the surface waves are more sensitive to the crustal structure. 
(a) Geodynamically-constrained inversion

(surface \& body waves)

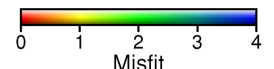

TA

$\mathrm{EH}$

MM
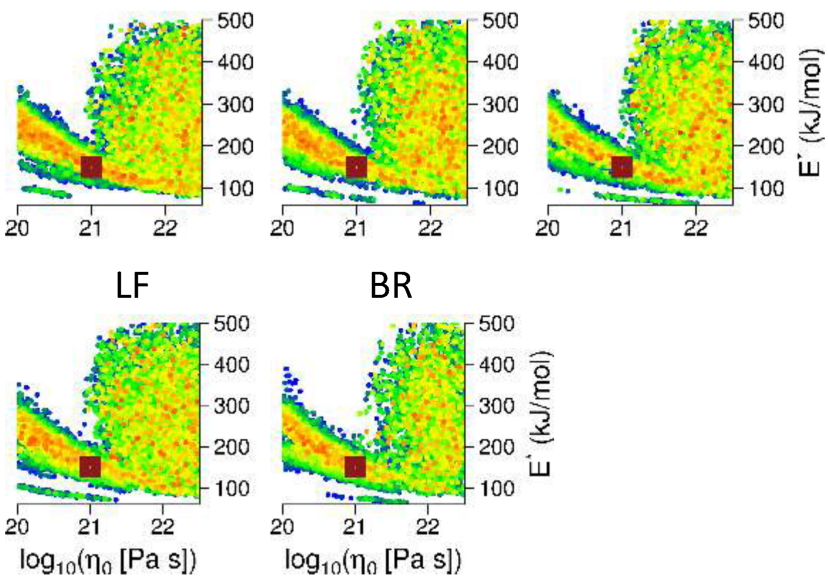

BR

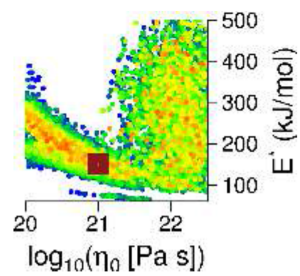

(b) Geodynamically-constrained inversion: four events (body waves only)

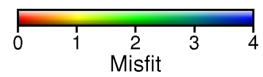

TA

$\mathrm{EH}$
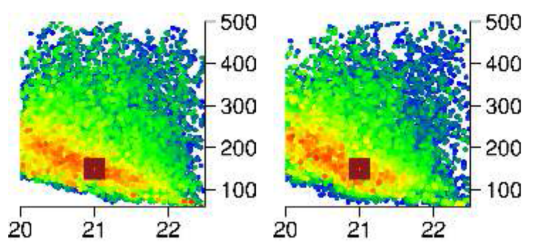

MM

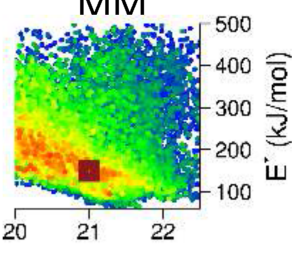

$\mathrm{LF}$

BR
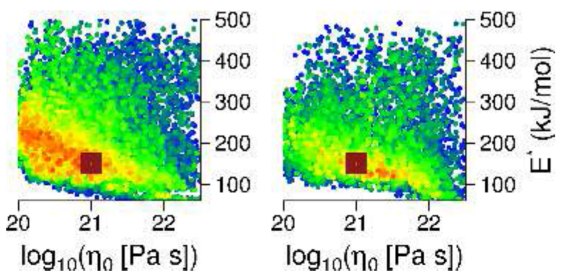

Figure B1. Inversion results considering different mineralogical compositions using (a) surface waves and body waves arrival times for a single located event, and (b) body waves arrival time from four located impact events. The figures show $\eta_{0}\left[\mathrm{in}\left(\log _{10}\right)\right]$ as a function of $E^{*}\left(\mathrm{in} \mathrm{kJ} \mathrm{mol}^{-1}\right)$ for all the models accepted by the Bayesian algorithm (coloured dots). Red and blue colours are small and large misfit values, respectively. The dark red squares correspond to the values to retrieve, which are equal to $\eta_{0}=$ $10^{21} \mathrm{~Pa} \mathrm{~s}$ and $E^{*}=150 \mathrm{~kJ} \mathrm{~mol}^{-1}$. TA stands for the mineralogical composition of Taylor (2013), EH for Sanloup et al. (1999), MM for Mohapatra \& Murty (2003), LF for Lodders \& Fegley (1997) and BR for Brasser et al. (2018). 
(a) Geodynamically-constrained inversion

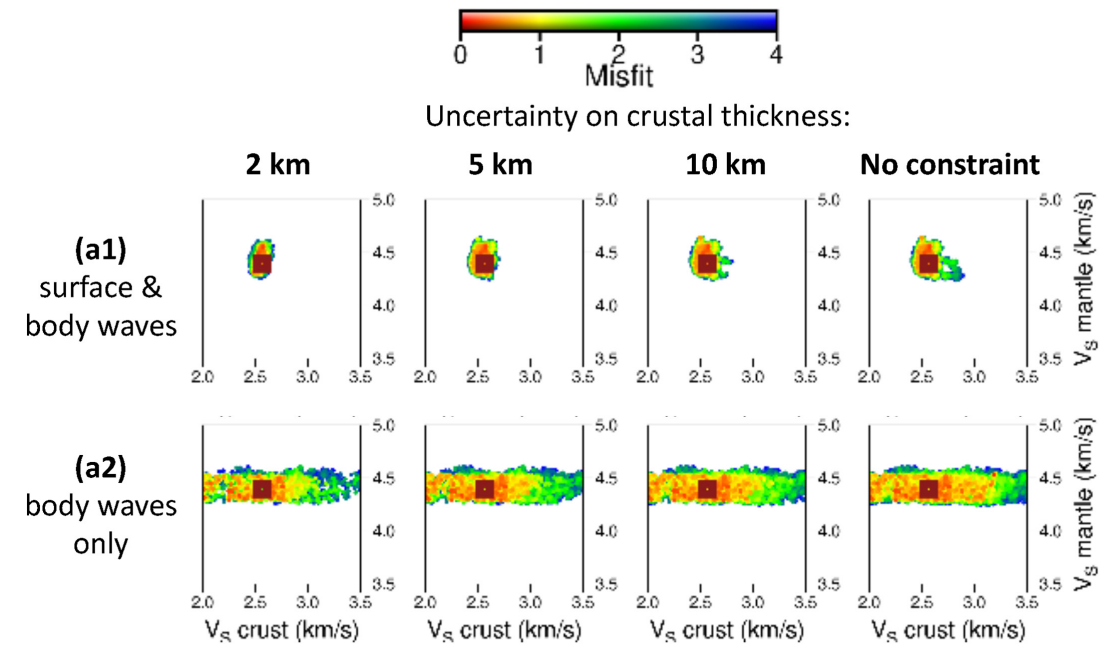

(b) Classical inversion

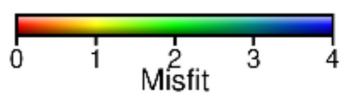

Uncertainty on crustal thickness:

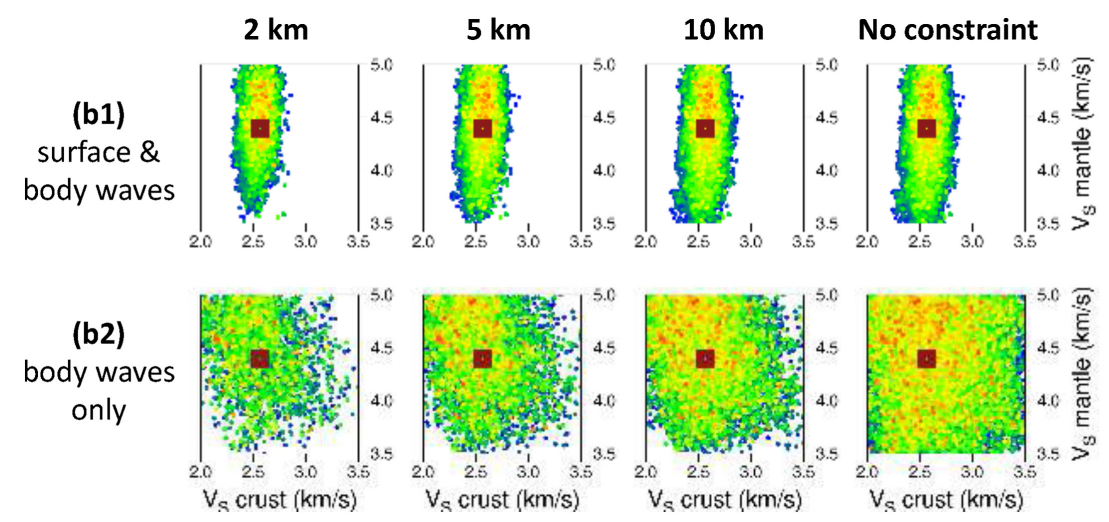

Figure C1. Inversion results for the (a) geodynamically constrained and the (b) classical inversions, considering and $a$ priori uncertainties of \pm 2 , \pm 5 and $\pm 10 \mathrm{~km}$ around the true value of the crustal thickness. The last column corresponds to the case where no constraints are applied. (a1) and (b1) are the results using both body waves and surface waves arrival times, and (a2) and (b2) are the results using body waves arrival times only. The figures show $V_{S}$ (in $\mathrm{km} \mathrm{s}^{-1}$ ) at the top of the mantle as a function of $V_{S}$ (in $\mathrm{km} \mathrm{s}^{-1}$ ) at the bottom of the crust, for all the models accepted by the Bayesian algorithm (coloured dots). Red and blue colours indicate small and large misfit values, respectively. The dark red squares correspond to the values to retrieve, corresponding to $\eta_{0}=10^{21}$ Pa $\mathrm{s}$ and $E^{*}=150 \mathrm{~kJ} \mathrm{~mol}^{-1}$. 


\section{Key words}

\begin{tabular}{|c|c|c|}
\hline COMPOSITION and PHYSICAL PROPERTIES & Seismic cycle & Instability analysis \\
\hline Composition and structure of the continental crust & Space geodetic surveys & Interferometry \\
\hline Composition and structure of the core & Tides and planetary waves & Inverse theory \\
\hline Composition and structure of the mantle & Time variable gravity & Joint inversion \\
\hline Composition and structure of the oceanic crust & Transient deformation & Neural networks, fuzzy logic \\
\hline Composition of the planets & & Non-linear differential equations \\
\hline Creep and deformation & GEOGRAPHIC LOCATION & Numerical approximations and analysis \\
\hline Defects & Africa & Numerical modelling \\
\hline Elasticity and anelasticity & Antarctica & Numerical solutions \\
\hline Electrical properties & Arctic region & Persistence, memory, correlations, clustering \\
\hline Equations of state & Asia & Probabilistic forecasting \\
\hline Fault zone rheology & Atlantic Ocean & Probability distributions \\
\hline Fracture and flow & Australia & Self-organization \\
\hline Friction & Europe & Spatial analysis \\
\hline High-pressure behaviour & Indian Ocean & Statistical methods \\
\hline Magnetic properties & Japan & Thermobarometry \\
\hline Microstructure & New Zealand & Time-series analysis \\
\hline Permeability and porosity & North America & Tomography \\
\hline Phase transitions & Pacific Ocean & Waveform inversion \\
\hline Plasticity, diffusion, and creep & South America & Wavelet transform \\
\hline GENERAL SUBJECTS & GEOMAGNETISM and ELECTROMAGNETISM & PLANETS \\
\hline Core & Archaeomagnetism & Planetary interiors \\
\hline Gas and hydrate systems & Biogenic magnetic minerals & Planetary volcanism \\
\hline Geomechanics & Controlled source electromagnetics (CSEM) & \\
\hline Geomorphology & Dynamo: theories and simulations & SEISMOLOGY \\
\hline Glaciology & Electrical anisotropy & Acoustic properties \\
\hline Heat flow & Electrical resistivity tomography (ERT) & Body waves \\
\hline Hydrogeophysics & Electromagnetic theory & Coda waves \\
\hline Hydrology & Environmental magnetism & Computational seismology \\
\hline Hydrothermal systems & Geomagnetic excursions & Controlled source seismology \\
\hline Infrasound & Geomagnetic induction & Crustal imaging \\
\hline Instrumental noise & Ground penetrating radar & Earthquake dynamics \\
\hline Ionosphere/atmosphere interactions & Magnetic anomalies: modelling and & Earthquake early warning \\
\hline Ionosphere/magnetosphere interactions & interpretation & Earthquake ground motions \\
\hline Mantle processes & Magnetic fabrics and anisotropy & Earthquake hazards \\
\hline Ocean drilling & Magnetic field variations through time & Earthquake interaction, forecasting, \\
\hline Structure of the Earth & Magnetic mineralogy and petrology & and prediction \\
\hline Thermochronology & Magnetostratigraphy & Earthquake monitoring and test-ban \\
\hline Tsunamis & Magnetotellurics & treaty verification \\
\hline Ultra-high pressure metamorphism & Marine electromagnetics & Earthquake source observations \\
\hline Ultra-high temperature metamorphism & Marine magnetics and palaeomagnetics & Guided waves \\
\hline & Non-linear electromagnetics & Induced seismicity \\
\hline GEODESY and GRAVITY & Palaeointensity & Interface waves \\
\hline Acoustic-gravity waves & Palaeomagnetic secular variation & Palaeoseismology \\
\hline Earth rotation variations & Palaeomagnetism & Rheology and friction of fault zones \\
\hline Geodetic instrumentation & Rapid time variations & Rotational seismology \\
\hline Geopotential theory & Remagnetization & Seismic anisotropy \\
\hline Global change from geodesy & Reversals: process, time scale, & Seismic attenuation \\
\hline Gravity anomalies and Earth structure & magnetostratigraphy & Seismic instruments \\
\hline Loading of the Earth & Rock and mineral magnetism & Seismic interferometry \\
\hline Lunar and planetary geodesy and gravity & Satellite magnetics & Seismicity and tectonics \\
\hline Plate motions & & Seismic noise \\
\hline Radar interferometry & GEOPHYSICAL METHODS & Seismic tomography \\
\hline Reference systems & Downhole methods & Site effects \\
\hline Satellite geodesy & Fourier analysis & Statistical seismology \\
\hline Satellite gravity & Fractals and multifractals & Surface waves and free oscillations \\
\hline Sea level change & Image processing & Theoretical seismology \\
\hline
\end{tabular}


Tsunami warning

Volcano seismology

Wave propagation

Wave scattering and diffraction

\section{TECTONOPHYSICS}

Backarc basin processes

Continental margins: convergent

Continental margins: divergent

Continental margins: transform

Continental neotectonics

Continental tectonics: compressional

Continental tectonics: extensional

Continental tectonics: strike-slip and transform

Cratons

Crustal structure

Diapirism

Dynamics: convection currents, and mantle plumes

Dynamics: gravity and tectonics

Dynamics: seismotectonics

Dynamics and mechanics of faulting

Dynamics of lithosphere and mantle

Folds and folding

Fractures, faults, and high strain deformation zones

Heat generation and transport
Hotspots

Impact phenomena

Intra-plate processes

Kinematics of crustal and mantle deformation

Large igneous provinces

Lithospheric flexure

Mechanics, theory, and modelling

Microstructures

Mid-ocean ridge processes

Neotectonics

Obduction tectonics

Oceanic hotspots and intraplate volcanism

Oceanic plateaus and microcontinents

Oceanic transform and fracture zone processes

Paleoseismology

Planetary tectonics

Rheology: crust and lithosphere

Rheology: mantle

Rheology and friction of fault zones

Sedimentary basin processes

Subduction zone processes

Submarine landslides

Submarine tectonics and volcanism

Tectonics and climatic interactions

Tectonics and landscape evolution

Transform faults

Volcanic arc processes
VOLCANOLOGY

Atmospheric effects (volcano)

Calderas

Effusive volcanism

Eruption mechanisms and flow emplacement

Experimental volcanism

Explosive volcanism

Lava rheology and morphology

Magma chamber processes

Magma genesis and partial melting

Magma migration and fragmentation

Mud volcanism

Physics and chemistry of magma bodies

Physics of magma and magma bodies

Planetary volcanism

Pluton emplacement

Remote sensing of volcanoes

Subaqueous volcanism

Tephrochronology

Volcanic gases

Volcanic hazards and risks

Volcaniclastic deposits

Volcano/climate interactions

Volcano monitoring

Volcano seismology 